\title{
Estimating Systematic Error and Uncertainty in Ab Initio Thermochemistry: II. ATOMIC(hc) Enthalpies of Formation for a Large Set of Hydrocarbons
}

Dirk Bakowies

November 10, 2019

\section{Supporting Information}

Please note that all literature references and references to Figures, Tables, and Sections with numerical label (without preceding "S") apply to the bibliography and material included in the paper. 


\section{List of Figures}

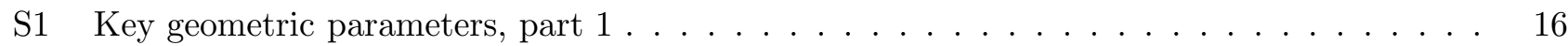

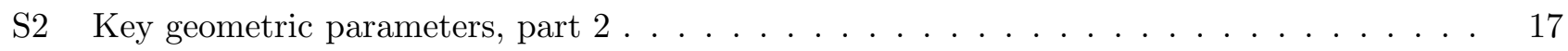

S3 Key geometric parameters, part $3 \ldots \ldots \ldots \ldots \ldots$

S4 Key geometric parameters, part $4 \ldots \ldots \ldots \ldots \ldots$

S5 Component corrections for the complete set of 161 hydrocarbons. . . . . . . . . . . 53

\section{List of Tables}

$\mathrm{S} 1 \quad$ List of molecules $\ldots \ldots \ldots \ldots \ldots \ldots \ldots \ldots \ldots$

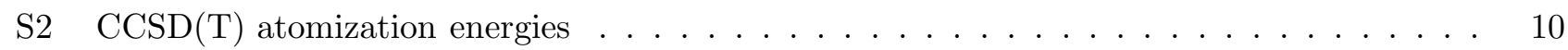

S3 Bond lengths: Comparison to accurate literature data . . . . . . . . . . . . . . . 20

S4 Atomization energy corrections for MP2 geometry errors . . . . . . . . . . . . . . 21

S5 Zero-point energies of molecules with first-row atoms (HCNOF): Comparison to literature

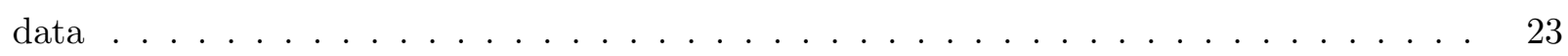

S6 Thermal enthalpy increments . . . . . . . . . . . . . . . . . 26

S7 Auxiliary data for atomization energies and enthalpies of formation . . . . . . . . . . . 29

S8 Enthalpies of formation $(298.15 \mathrm{~K}) \ldots \ldots \ldots \ldots \ldots$

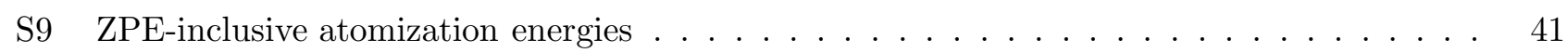

S10 Best available theoretical data . . . . . . . . . . . . . . . . . . 47 
Table S1: List of molecules

\begin{tabular}{|c|c|c|c|c|c|c|c|}
\hline MID & & Molecule & Point Group & ${ }^{3} \mathrm{C}-\mathrm{H}$ & ${ }^{3} \mathrm{C}-\mathrm{C}^{3}$ & ${ }^{2} \mathrm{C}=\mathrm{C}^{2}$ & ${ }^{1} \mathrm{C} \equiv \mathrm{C}^{1}$ \\
\hline 1 & $\mathrm{CH}_{4}$ & methane & $\mathrm{T}_{\mathrm{d}}$ & 4 & 0 & 0 & 0 \\
\hline 2 & $\mathrm{C}_{2} \mathrm{H}_{2}$ & acetylene & $\mathrm{D}_{\infty \mathrm{h}}$ & 2 & 0 & 0 & 1 \\
\hline 3 & $\mathrm{C}_{2} \mathrm{H}_{4}$ & ethylene & $\mathrm{D}_{2 \mathrm{~h}}$ & 4 & 0 & 1 & 0 \\
\hline 4 & $\mathrm{C}_{2} \mathrm{H}_{6}$ & ethane & $\mathrm{D}_{3 \mathrm{~d}}$ & 6 & 1 & 0 & 0 \\
\hline 5 & $\mathrm{C}_{3} \mathrm{H}_{4}$ & allene & $\mathrm{D}_{2 \mathrm{~d}}$ & 4 & 0 & 2 & 0 \\
\hline 6 & $\mathrm{C}_{3} \mathrm{H}_{4}$ & cyclopropene & $\mathrm{C}_{2 \mathrm{v}}$ & 4 & 2 & 1 & 0 \\
\hline 7 & $\mathrm{C}_{3} \mathrm{H}_{4}$ & propyne & $\mathrm{C}_{3 \mathrm{v}}$ & 4 & 1 & 0 & 1 \\
\hline 8 & $\mathrm{C}_{3} \mathrm{H}_{6}$ & cyclopropane & $\mathrm{D}_{3 \mathrm{~h}}$ & 6 & 3 & 0 & 0 \\
\hline 9 & $\mathrm{C}_{3} \mathrm{H}_{6}$ & propene & $\mathrm{C}_{\mathrm{s}}$ & 6 & 1 & 1 & 0 \\
\hline 10 & $\mathrm{C}_{3} \mathrm{H}_{8}$ & propane & $\mathrm{C}_{2 \mathrm{v}}$ & 8 & 2 & 0 & 0 \\
\hline 11 & $\mathrm{C}_{4} \mathrm{H}_{2}$ & 1,3-butadiyne & $\mathrm{D}_{\infty \mathrm{h}}$ & 2 & 1 & 0 & 2 \\
\hline 12 & $\mathrm{C}_{4} \mathrm{H}_{4}$ & bicyclo[1.1.0]but-1(3)-ene & $\mathrm{C}_{2 \mathrm{v}}$ & 4 & 4 & 1 & 0 \\
\hline 13 & $\mathrm{C}_{4} \mathrm{H}_{4}$ & butatriene & $\mathrm{D}_{2 \mathrm{~h}}$ & 4 & 0 & 3 & 0 \\
\hline 14 & $\mathrm{C}_{4} \mathrm{H}_{4}$ & cyclobutadiene & $\mathrm{D}_{2 \mathrm{~h}}$ & 4 & 2 & 2 & 0 \\
\hline 15 & $\mathrm{C}_{4} \mathrm{H}_{4}$ & ethynylethene & $\mathrm{C}_{\mathrm{s}}$ & 4 & 1 & 1 & 1 \\
\hline 16 & $\mathrm{C}_{4} \mathrm{H}_{4}$ & methylenecyclopropene & $\mathrm{C}_{2 \mathrm{v}}$ & 4 & 2 & 2 & 0 \\
\hline 17 & $\mathrm{C}_{4} \mathrm{H}_{4}$ & tetrahedrane & $\mathrm{T}_{\mathrm{d}}$ & 4 & 6 & 0 & 0 \\
\hline 18 & $\mathrm{C}_{4} \mathrm{H}_{6}$ & 1,2-butadiene & $\mathrm{C}_{\mathrm{s}}$ & 6 & 1 & 2 & 0 \\
\hline 19 & $\mathrm{C}_{4} \mathrm{H}_{6}$ & 1,3-butadiene & $\mathrm{C}_{2 \mathrm{~h}}$ & 6 & 1 & 2 & 0 \\
\hline 20 & $\mathrm{C}_{4} \mathrm{H}_{6}$ & 2-butyne & $\mathrm{D}_{3 \mathrm{~h}}$ & 6 & 2 & 0 & 1 \\
\hline 21 & $\mathrm{C}_{4} \mathrm{H}_{6}$ & bicyclo[1.1.0]butane & $\mathrm{C}_{2 \mathrm{v}}$ & 6 & 5 & 0 & 0 \\
\hline 22 & $\mathrm{C}_{4} \mathrm{H}_{6}$ & cyclobutene & $\mathrm{C}_{2 \mathrm{v}}$ & 6 & 3 & 1 & 0 \\
\hline 23 & $\mathrm{C}_{4} \mathrm{H}_{6}$ & methylenecyclopropane & $\mathrm{C}_{2 \mathrm{v}}$ & 6 & 3 & 1 & 0 \\
\hline 24 & $\mathrm{C}_{4} \mathrm{H}_{8}$ & cyclobutane & $\mathrm{C}_{2 \mathrm{v}}$ & 8 & 4 & 0 & 0 \\
\hline 25 & $\mathrm{C}_{4} \mathrm{H}_{8}$ & isobutene & $\mathrm{C}_{2 \mathrm{v}}$ & 8 & 2 & 1 & 0 \\
\hline 26 & $\mathrm{C}_{4} \mathrm{H}_{10}$ & isobutane & $\mathrm{C}_{3 \mathrm{v}}$ & 10 & 3 & 0 & 0 \\
\hline 27 & $\mathrm{C}_{4} \mathrm{H}_{10}$ & $n$-butane & $\mathrm{C}_{2 \mathrm{~h}}$ & 10 & 3 & 0 & 0 \\
\hline 28 & $\mathrm{C}_{5} \mathrm{H}_{4}$ & 1,4-pentadiyne & $\mathrm{C}_{2 \mathrm{v}}$ & 4 & 2 & 0 & 2 \\
\hline 29 & $\mathrm{C}_{5} \mathrm{H}_{4}$ & pentatetraene & $\mathrm{D}_{2 \mathrm{~d}}$ & 4 & 0 & 4 & 0 \\
\hline
\end{tabular}


Table S1, contd.

\begin{tabular}{|c|c|c|c|c|c|c|c|}
\hline MID & & Molecule & Point Group & ${ }^{3} \mathrm{C}-\mathrm{H}$ & ${ }^{3} \mathrm{C}-\mathrm{C}^{3}$ & ${ }^{2} \mathrm{C}=\mathrm{C}^{2}$ & ${ }^{1} \mathrm{C} \equiv \mathrm{C}^{1}$ \\
\hline 30 & $\mathrm{C}_{5} \mathrm{H}_{4}$ & spiropentadiene & $\mathrm{D}_{2 \mathrm{~d}}$ & 4 & 4 & 2 & 0 \\
\hline 31 & $\mathrm{C}_{5} \mathrm{H}_{6}$ & [1.1.1]propellane & $\mathrm{D}_{3 \mathrm{~h}}$ & 6 & 7 & 0 & 0 \\
\hline 32 & $\mathrm{C}_{5} \mathrm{H}_{6}$ & 1,3-cyclopentadiene & $\mathrm{C}_{2 \mathrm{v}}$ & 6 & 3 & 2 & 0 \\
\hline 33 & $\mathrm{C}_{5} \mathrm{H}_{6}$ & 3-methylenecyclobutene & $\mathrm{C}_{\mathrm{s}}$ & 6 & 3 & 2 & 0 \\
\hline 34 & $\mathrm{C}_{5} \mathrm{H}_{6}$ & bicyclo[2.1.0]pent-2-ene & $\mathrm{C}_{\mathrm{s}}$ & 6 & 5 & 1 & 0 \\
\hline 35 & $\mathrm{C}_{5} \mathrm{H}_{6}$ & cyclopropylacetylene & $\mathrm{C}_{\mathrm{s}}$ & 6 & 4 & 0 & 1 \\
\hline 36 & $\mathrm{C}_{5} \mathrm{H}_{8}$ & 1,4-pentadiene & $\mathrm{C}_{2}$ & 8 & 2 & 2 & 0 \\
\hline 37 & $\mathrm{C}_{5} \mathrm{H}_{8}$ & isoprene & $\mathrm{C}_{\mathrm{s}}$ & 8 & 2 & 2 & 0 \\
\hline 38 & $\mathrm{C}_{5} \mathrm{H}_{8}$ & spiropentane & $\mathrm{D}_{2 \mathrm{~d}}$ & 8 & 6 & 0 & 0 \\
\hline 39 & $\mathrm{C}_{5} \mathrm{H}_{10}$ & cyclopentane & $\mathrm{C}_{\mathrm{s}}{ }^{a}$ & 10 & 5 & 0 & 0 \\
\hline 40 & $\mathrm{C}_{5} \mathrm{H}_{12}$ & neopentane & $\mathrm{T}_{\mathrm{d}}$ & 12 & 4 & 0 & 0 \\
\hline 41 & $\mathrm{C}_{5} \mathrm{H}_{12}$ & $n$-pentane & $\mathrm{C}_{2 \mathrm{v}}$ & 12 & 4 & 0 & 0 \\
\hline 42 & $\mathrm{C}_{6} \mathrm{H}_{2}$ & 1,3,5-hexatriyne & $\mathrm{D}_{\infty \mathrm{h}}$ & 2 & 2 & 0 & 3 \\
\hline 43 & $\mathrm{C}_{6} \mathrm{H}_{4}$ & butalene & $\mathrm{D}_{2 \mathrm{~h}}$ & 4 & 4 & 3 & 0 \\
\hline 44 & $\mathrm{C}_{6} \mathrm{H}_{4}$ & cis-3-hexene-1,5-diyne & $\mathrm{C}_{2 \mathrm{v}}$ & 4 & 2 & 1 & 2 \\
\hline 45 & $\mathrm{C}_{6} \mathrm{H}_{4}$ & hexapentaene & $\mathrm{D}_{2 \mathrm{~h}}$ & 4 & 0 & 5 & 0 \\
\hline 46 & $\mathrm{C}_{6} \mathrm{H}_{4}$ & ortho-benzyne & $\mathrm{C}_{2 \mathrm{v}}$ & 4 & 3 & 2 & 1 \\
\hline 47 & $\mathrm{C}_{6} \mathrm{H}_{4}$ & trans-3-hexene-1,5-diyne & $\mathrm{C}_{2 \mathrm{~h}}$ & 4 & 2 & 1 & 2 \\
\hline 48 & $\mathrm{C}_{6} \mathrm{H}_{4}$ & triafulvalene & $\mathrm{D}_{2 \mathrm{~h}}$ & 4 & 4 & 3 & 0 \\
\hline 49 & $\mathrm{C}_{6} \mathrm{H}_{6}$ & 2,4-hexadiyne & $\mathrm{D}_{3 \mathrm{~d}}$ & 6 & 3 & 0 & 2 \\
\hline 50 & $\mathrm{C}_{6} \mathrm{H}_{6}$ & 3,3'-bicyclopropenyl & $\mathrm{C}_{2 \mathrm{~h}}$ & 6 & 5 & 2 & 0 \\
\hline 51 & $\mathrm{C}_{6} \mathrm{H}_{6}$ & 3,4-dimethylenecyclobutene & $\mathrm{C}_{2 \mathrm{v}}$ & 6 & 3 & 3 & 0 \\
\hline 52 & $\mathrm{C}_{6} \mathrm{H}_{6}$ & [3] radialene & $\mathrm{D}_{3 \mathrm{~h}}$ & 6 & 3 & 3 & 0 \\
\hline 53 & $\mathrm{C}_{6} \mathrm{H}_{6}$ & benzene & $\mathrm{D}_{6 \mathrm{~h}}$ & 6 & 3 & 3 & 0 \\
\hline 54 & $\mathrm{C}_{6} \mathrm{H}_{6}$ & benzvalene & $\mathrm{C}_{2 \mathrm{v}}$ & 6 & 7 & 1 & 0 \\
\hline 55 & $\mathrm{C}_{6} \mathrm{H}_{6}$ & Dewar benzene & $\mathrm{C}_{2 \mathrm{v}}$ & 6 & 5 & 2 & 0 \\
\hline 56 & $\mathrm{C}_{6} \mathrm{H}_{6}$ & fulvene & $\mathrm{C}_{2 \mathrm{v}}$ & 6 & 3 & 3 & 0 \\
\hline 57 & $\mathrm{C}_{6} \mathrm{H}_{6}$ & prismane & $\mathrm{D}_{3 \mathrm{~h}}$ & 6 & 9 & 0 & 0 \\
\hline 58 & $\mathrm{C}_{6} \mathrm{H}_{8}$ & 1,3-cyclohexadiene & $\mathrm{C}_{2}$ & 8 & 4 & 2 & 0 \\
\hline 59 & $\mathrm{C}_{6} \mathrm{H}_{8}$ & 1,4-cyclohexadiene & $\mathrm{D}_{2 \mathrm{~h}}$ & 8 & 4 & 2 & 0 \\
\hline
\end{tabular}


Table S1, contd.

\begin{tabular}{|c|c|c|c|c|c|c|c|}
\hline MID & & Molecule & Point Group & ${ }^{3} \mathrm{C}-\mathrm{H}$ & ${ }^{3} \mathrm{C}-\mathrm{C}^{3}$ & ${ }^{2} \mathrm{C}=\mathrm{C}^{2}$ & ${ }^{1} \mathrm{C} \equiv \mathrm{C}^{1}$ \\
\hline 60 & $\mathrm{C}_{6} \mathrm{H}_{8}$ & trans-1,3,5-hexatriene & $\mathrm{C}_{2 \mathrm{~h}}$ & 8 & 2 & 3 & 0 \\
\hline 61 & $\mathrm{C}_{6} \mathrm{H}_{10}$ & cyclohexene & $\mathrm{C}_{2}$ & 10 & 5 & 1 & 0 \\
\hline 62 & $\mathrm{C}_{6} \mathrm{H}_{12}$ & cyclohexane & $\mathrm{D}_{3 \mathrm{~d}}$ & 12 & 6 & 0 & 0 \\
\hline 63 & $\mathrm{C}_{6} \mathrm{H}_{14}$ & 3-methylpentane & $\mathrm{C}_{\mathrm{s}}$ & 14 & 5 & 0 & 0 \\
\hline 64 & $\mathrm{C}_{6} \mathrm{H}_{14}$ & $n$-hexane & $\mathrm{C}_{2 \mathrm{~h}}$ & 14 & 5 & 0 & 0 \\
\hline 65 & $\mathrm{C}_{7} \mathrm{H}_{4}$ & 3,3-diethynylcyclopropene & $\mathrm{C}_{2 \mathrm{v}}$ & 4 & 4 & 1 & 2 \\
\hline 66 & $\mathrm{C}_{7} \mathrm{H}_{4}$ & heptahexaene & $\mathrm{D}_{2 \mathrm{~d}}$ & 4 & 0 & 6 & 0 \\
\hline 67 & $\mathrm{C}_{7} \mathrm{H}_{6}$ & 1,1-diethynylcyclopropane & $\mathrm{C}_{2 \mathrm{v}}$ & 6 & 5 & 0 & 2 \\
\hline 68 & $\mathrm{C}_{7} \mathrm{H}_{6}$ & $1,3,5$-norcaratriene & $\mathrm{C}_{2 \mathrm{v}}$ & 6 & 5 & 3 & 0 \\
\hline 69 & $\mathrm{C}_{7} \mathrm{H}_{6}$ & fulvenallene & $\mathrm{C}_{2 \mathrm{v}}$ & 6 & 3 & 4 & 0 \\
\hline 70 & $\mathrm{C}_{7} \mathrm{H}_{6}$ & spiro[2.4] hepta-1,4,6-triene & $\mathrm{C}_{2 \mathrm{v}}$ & 6 & 5 & 3 & 0 \\
\hline 71 & $\mathrm{C}_{7} \mathrm{H}_{8}$ & 1,3,5-cycloheptatriene & $\mathrm{C}_{\mathrm{s}}$ & 8 & 4 & 3 & 0 \\
\hline 72 & $\mathrm{C}_{7} \mathrm{H}_{8}$ & 2,5-norbornadiene & $\mathrm{C}_{2 \mathrm{v}}$ & 8 & 6 & 2 & 0 \\
\hline 73 & $\mathrm{C}_{7} \mathrm{H}_{8}$ & norcaradiene & $\mathrm{C}_{\mathrm{s}}$ & 8 & 6 & 2 & 0 \\
\hline 74 & $\mathrm{C}_{7} \mathrm{H}_{8}$ & quadricyclane & $\mathrm{C}_{2 \mathrm{v}}$ & 8 & 10 & 0 & 0 \\
\hline 75 & $\mathrm{C}_{7} \mathrm{H}_{8}$ & spiro[2.4]hepta-4,6-diene & $\mathrm{C}_{2 \mathrm{v}}$ & 8 & 6 & 2 & 0 \\
\hline 76 & $\mathrm{C}_{7} \mathrm{H}_{8}$ & toluene & $\mathrm{C}_{\mathrm{s}}$ & 8 & 4 & 3 & 0 \\
\hline 77 & $\mathrm{C}_{7} \mathrm{H}_{12}$ & norbornane & $\mathrm{C}_{2 \mathrm{v}}$ & 12 & 8 & 0 & 0 \\
\hline 78 & $\mathrm{C}_{7} \mathrm{H}_{16}$ & $n$-heptane & $\mathrm{C}_{2 \mathrm{v}}$ & 16 & 6 & 0 & 0 \\
\hline 79 & $\mathrm{C}_{8} \mathrm{H}_{2}$ & 1,3,5,7-octatetrayne & $\mathrm{D}_{\infty \mathrm{h}}$ & 2 & 3 & 0 & 4 \\
\hline 80 & $\mathrm{C}_{8} \mathrm{H}_{4}$ & 1,2,3,5,6,7-cyclooctahexaene & $\mathrm{C}_{2 \mathrm{~h}}$ & 4 & 2 & 6 & 0 \\
\hline 81 & $\mathrm{C}_{8} \mathrm{H}_{4}$ & 1,5-cyclooctadiene-3,7-diyne & $\mathrm{D}_{2 \mathrm{~h}}$ & 4 & 4 & 2 & 2 \\
\hline 82 & $\mathrm{C}_{8} \mathrm{H}_{4}$ & octaheptaene & $\mathrm{D}_{2 \mathrm{~h}}$ & 4 & 0 & 7 & 0 \\
\hline 83 & $\mathrm{C}_{8} \mathrm{H}_{6}$ & benzocyclobutadiene & $\mathrm{C}_{2 \mathrm{v}}$ & 6 & 5 & 4 & 0 \\
\hline 84 & $\mathrm{C}_{8} \mathrm{H}_{6}$ & bis(cyclopropa)benzene & $\mathrm{D}_{2 \mathrm{~h}}$ & 6 & 7 & 3 & 0 \\
\hline 85 & $\mathrm{C}_{8} \mathrm{H}_{6}$ & pentalene & $\mathrm{C}_{2 \mathrm{~h}}$ & 6 & 5 & 4 & 0 \\
\hline 86 & $\mathrm{C}_{8} \mathrm{H}_{8}$ & 1,3,5,7-cyclooctatetraene & $\mathrm{D}_{2 \mathrm{~d}}$ & 8 & 4 & 4 & 0 \\
\hline 87 & $\mathrm{C}_{8} \mathrm{H}_{8}$ & benzocyclobutene & $\mathrm{C}_{2 \mathrm{v}}$ & 8 & 6 & 3 & 0 \\
\hline 88 & $\mathrm{C}_{8} \mathrm{H}_{8}$ & cubane & $\mathrm{O}_{\mathrm{h}}$ & 8 & 12 & 0 & 0 \\
\hline 89 & $\mathrm{C}_{8} \mathrm{H}_{8}$ & para-xylylene & $\mathrm{D}_{2 \mathrm{~h}}$ & 8 & 4 & 4 & 0 \\
\hline
\end{tabular}


Table S1, contd.

\begin{tabular}{|c|c|c|c|c|c|c|c|}
\hline MID & & Molecule & Point Group & ${ }^{3} \mathrm{C}-\mathrm{H}$ & ${ }^{3} \mathrm{C}-\mathrm{C}^{3}$ & ${ }^{2} \mathrm{C}=\mathrm{C}^{2}$ & ${ }^{1} \mathrm{C} \equiv \mathrm{C}^{1}$ \\
\hline 90 & $\mathrm{C}_{8} \mathrm{H}_{8}$ & styrene & $\mathrm{C}_{1}$ & 8 & 4 & 4 & 0 \\
\hline 91 & $\mathrm{C}_{8} \mathrm{H}_{10}$ & all-trans-octatetraene & $\mathrm{C}_{2 \mathrm{~h}}$ & 10 & 3 & 4 & 0 \\
\hline 92 & $\mathrm{C}_{8} \mathrm{H}_{14}$ & bicyclo[2.2.2] octane & $\mathrm{D}_{3}$ & 14 & 9 & 0 & 0 \\
\hline 93 & $\mathrm{C}_{8} \mathrm{H}_{18}$ & $n$-octane & $\mathrm{C}_{2 \mathrm{~h}}$ & 18 & 7 & 0 & 0 \\
\hline 94 & $\mathrm{C}_{9} \mathrm{H}_{20}$ & $n$-nonane & $\mathrm{C}_{2 \mathrm{v}}$ & 20 & 8 & 0 & 0 \\
\hline 95 & $\mathrm{C}_{10} \mathrm{H}_{2}$ & 1,3,5,7,9-decapentayne & $\mathrm{D}_{\infty \mathrm{h}}$ & 2 & 4 & 0 & 5 \\
\hline 96 & $\mathrm{C}_{10} \mathrm{H}_{4}$ & tetraethynylethene & $\mathrm{D}_{2 \mathrm{~h}}$ & 4 & 4 & 1 & 4 \\
\hline 97 & $\mathrm{C}_{10} \mathrm{H}_{8}$ & azulene & $\mathrm{C}_{2 \mathrm{v}}$ & 8 & 6 & 5 & 0 \\
\hline 98 & $\mathrm{C}_{10} \mathrm{H}_{8}$ & naphthalene & $\mathrm{D}_{2 \mathrm{~h}}$ & 8 & 6 & 5 & 0 \\
\hline 99 & $\mathrm{C}_{10} \mathrm{H}_{8}$ & pentafulvalene & $\mathrm{D}_{2 \mathrm{~h}}$ & 8 & 6 & 5 & 0 \\
\hline 100 & $\mathrm{C}_{10} \mathrm{H}_{10}$ & 1,4-dicyclopropyl-1,3-butadiyne & $\mathrm{C}_{2}$ & 10 & 9 & 0 & 2 \\
\hline 101 & $\mathrm{C}_{10} \mathrm{H}_{10}$ & 1-butynylbenzene & $\mathrm{C}_{\mathrm{s}}$ & 10 & 6 & 3 & 1 \\
\hline 102 & $\mathrm{C}_{10} \mathrm{H}_{10}$ & 1-methylene-2-phenylcyclopropane & $\mathrm{C}_{1}$ & 10 & 7 & 4 & 0 \\
\hline 103 & $\mathrm{C}_{10} \mathrm{H}_{10}$ & 3,4-bis(methylene)bicyclo[4.2.0]octa-1,5-diene & $\mathrm{C}_{2}$ & 10 & 7 & 4 & 0 \\
\hline 104 & $\mathrm{C}_{10} \mathrm{H}_{10}$ & basketene & $\mathrm{C}_{3 \mathrm{v}}$ & 10 & 13 & 1 & 0 \\
\hline 105 & $\mathrm{C}_{10} \mathrm{H}_{10}$ & benzo[1,2:4,5]dicyclobutene & $\mathrm{D}_{2 \mathrm{~h}}$ & 10 & 9 & 3 & 0 \\
\hline 106 & $\mathrm{C}_{10} \mathrm{H}_{10}$ & bullvalene & $\mathrm{C}_{3 \mathrm{v}}$ & 10 & 9 & 3 & 0 \\
\hline 107 & $\mathrm{C}_{10} \mathrm{H}_{10}$ & diademane & $\mathrm{C}_{3 \mathrm{v}}$ & 10 & 15 & 0 & 0 \\
\hline 108 & $\mathrm{C}_{10} \mathrm{H}_{10}$ & diisopropenyldiacetylene & $\mathrm{C}_{2 \mathrm{~h}}$ & 10 & 5 & 2 & 2 \\
\hline 109 & $\mathrm{C}_{10} \mathrm{H}_{10}$ & Nenitzescu's hydrocarbon & $\mathrm{C}_{\mathrm{s}}$ & 10 & 9 & 3 & 0 \\
\hline 110 & $\mathrm{C}_{10} \mathrm{H}_{10}$ & snoutene & $\mathrm{C}_{2 \mathrm{v}}$ & 10 & 13 & 1 & 0 \\
\hline 111 & $\mathrm{C}_{10} \mathrm{H}_{10}$ & triquinacene & $\mathrm{C}_{3 \mathrm{v}}$ & 10 & 9 & 3 & 0 \\
\hline 112 & $\mathrm{C}_{10} \mathrm{H}_{12}$ & all-trans-decapentaene & $\mathrm{C}_{2 \mathrm{~h}}$ & 12 & 4 & 5 & 0 \\
\hline 113 & $\mathrm{C}_{10} \mathrm{H}_{16}$ & adamantane & $\mathrm{T}_{\mathrm{d}}$ & 16 & 12 & 0 & 0 \\
\hline 114 & $\mathrm{C}_{10} \mathrm{H}_{16}$ & perhydroquinacene & $\mathrm{C}_{3}$ & 16 & 12 & 0 & 0 \\
\hline 115 & $\mathrm{C}_{10} \mathrm{H}_{18}$ & cis-decalin & $\mathrm{C}_{2}$ & 18 & 11 & 0 & 0 \\
\hline 116 & $\mathrm{C}_{10} \mathrm{H}_{18}$ & trans-decalin & $\mathrm{C}_{2 \mathrm{~h}}$ & 18 & 11 & 0 & 0 \\
\hline 117 & $\mathrm{C}_{10} \mathrm{H}_{22}$ & $n$-decane & $\mathrm{C}_{2 \mathrm{~h}}$ & 22 & 9 & 0 & 0 \\
\hline 118 & $\mathrm{C}_{11} \mathrm{H}_{24}$ & $n$-undecane & $\mathrm{C}_{2 \mathrm{v}}$ & 24 & 10 & 0 & 0 \\
\hline 119 & $\mathrm{C}_{12} \mathrm{H}_{6}$ & [3]circulene & $\mathrm{C}_{3 \mathrm{v}}$ & 6 & 9 & 6 & 0 \\
\hline
\end{tabular}


Table S1, contd.

\begin{tabular}{|c|c|c|c|c|c|c|c|}
\hline MID & & Molecule & Point Group & ${ }^{3} \mathrm{C}-\mathrm{H}$ & ${ }^{3} \mathrm{C}-\mathrm{C}^{3}$ & ${ }^{2} \mathrm{C}=\mathrm{C}^{2}$ & ${ }^{1} \mathrm{C} \equiv \mathrm{C}^{1}$ \\
\hline 120 & $\mathrm{C}_{12} \mathrm{H}_{6}$ & tricyclopropenylidenecyclopropane & $\mathrm{D}_{3 \mathrm{~h}}$ & 6 & 9 & 6 & 0 \\
\hline 121 & $\mathrm{C}_{12} \mathrm{H}_{8}$ & acenaphthylene & $\mathrm{C}_{2 \mathrm{v}}$ & 8 & 8 & 6 & 0 \\
\hline 122 & $\mathrm{C}_{12} \mathrm{H}_{8}$ & biphenylene & $\mathrm{D}_{2 \mathrm{~h}}$ & 8 & 8 & 6 & 0 \\
\hline 123 & $\mathrm{C}_{12} \mathrm{H}_{10}$ & biphenyl & $\mathrm{D}_{2}$ & 10 & 7 & 6 & 0 \\
\hline 124 & $\mathrm{C}_{12} \mathrm{H}_{12}$ & 1,2-benzo-1,3,7-cyclooctatriene & $\mathrm{C}_{1}$ & 12 & 8 & 5 & 0 \\
\hline 125 & $\mathrm{C}_{12} \mathrm{H}_{14}$ & all-trans-dodecahexaene & $\mathrm{C}_{2 \mathrm{~h}}$ & 14 & 5 & 6 & 0 \\
\hline 126 & $\mathrm{C}_{12} \mathrm{H}_{18}$ & hexamethylbenzene & $\mathrm{D}_{3 \mathrm{~d}}$ & 18 & 9 & 3 & 0 \\
\hline 127 & $\mathrm{C}_{12} \mathrm{H}_{26}$ & $n$-dodecane & $\mathrm{C}_{2 \mathrm{~h}}$ & 26 & 11 & 0 & 0 \\
\hline 128 & $\mathrm{C}_{13} \mathrm{H}_{10}$ & fluorene & $\mathrm{C}_{2 \mathrm{v}}$ & 10 & 9 & 6 & 0 \\
\hline 129 & $\mathrm{C}_{13} \mathrm{H}_{28}$ & $n$-tridecane & $\mathrm{C}_{2 \mathrm{v}}$ & 28 & 12 & 0 & 0 \\
\hline 130 & $\mathrm{C}_{14} \mathrm{H}_{8}$ & 9,10-didehydrophenanthrene & $\mathrm{C}_{2 \mathrm{v}}$ & 8 & 9 & 6 & 1 \\
\hline 131 & $\mathrm{C}_{14} \mathrm{H}_{8}$ & pyracyclene $^{c}$ & $\mathrm{D}_{2 \mathrm{~h}}$ & 8 & 10 & 7 & 0 \\
\hline 132 & $\mathrm{C}_{14} \mathrm{H}_{10}$ & anthracene & $\mathrm{D}_{2 \mathrm{~h}}$ & 10 & 9 & 7 & 0 \\
\hline 133 & $\mathrm{C}_{14} \mathrm{H}_{10}$ & phenanthrene & $\mathrm{C}_{2 \mathrm{v}}$ & 10 & 9 & 7 & 0 \\
\hline 134 & $\mathrm{C}_{14} \mathrm{H}_{20}$ & diamantane & $\mathrm{C}_{2 \mathrm{~h}}$ & 20 & 18 & 0 & 0 \\
\hline 135 & $\mathrm{C}_{14} \mathrm{H}_{30}$ & $n$-tetradecane & $\mathrm{C}_{2 \mathrm{~h}}$ & 30 & 13 & 0 & 0 \\
\hline 136 & $\mathrm{C}_{15} \mathrm{H}_{32}$ & $n$-pentadecane & $\mathrm{C}_{2 \mathrm{v}}$ & 32 & 14 & 0 & 0 \\
\hline 137 & $\mathrm{C}_{16} \mathrm{H}_{8}$ & [4]circulene & $\mathrm{C}_{4 \mathrm{v}}$ & 8 & 12 & 8 & 0 \\
\hline 138 & $\mathrm{C}_{16} \mathrm{H}_{10}$ & fluoranthene & $\mathrm{C}_{2 \mathrm{v}}$ & 10 & 11 & 8 & 0 \\
\hline 139 & $\mathrm{C}_{16} \mathrm{H}_{10}$ & pyrene & $\mathrm{D}_{2 \mathrm{~h}}$ & 10 & 11 & 8 & 0 \\
\hline 140 & $\mathrm{C}_{16} \mathrm{H}_{12}$ & [2.2]paracyclophanediene & $\mathrm{C}_{2 \mathrm{~h}}$ & 12 & 10 & 8 & 0 \\
\hline 141 & $\mathrm{C}_{16} \mathrm{H}_{16}$ & {$[2.2]$ metacyclophane } & $\mathrm{C}_{2 \mathrm{~h}}$ & 16 & 12 & 6 & 0 \\
\hline 142 & $\mathrm{C}_{16} \mathrm{H}_{16}$ & [2.2]metaparacyclophane & $\mathrm{C}_{\mathrm{s}}$ & 16 & 12 & 6 & 0 \\
\hline 143 & $\mathrm{C}_{16} \mathrm{H}_{16}$ & [2.2]paracyclophane & $\mathrm{D}_{2}$ & 16 & 12 & 6 & 0 \\
\hline 144 & $\mathrm{C}_{16} \mathrm{H}_{34}$ & $n$-hexadecane & $\mathrm{C}_{2 \mathrm{~h}}$ & 34 & 15 & 0 & 0 \\
\hline 145 & $\mathrm{C}_{18} \mathrm{H}_{12}$ & benzo $[\mathrm{c}]$ phenanthrene & $\mathrm{C}_{2}$ & 12 & 12 & 9 & 0 \\
\hline 146 & $\mathrm{C}_{18} \mathrm{H}_{12}$ & chrysene & $\mathrm{C}_{2 \mathrm{~h}}$ & 12 & 12 & 9 & 0 \\
\hline 147 & $\mathrm{C}_{18} \mathrm{H}_{12}$ & naphthacene & $\mathrm{D}_{2 \mathrm{~h}}$ & 12 & 12 & 9 & 0 \\
\hline 148 & $\mathrm{C}_{18} \mathrm{H}_{12}$ & triphenylene & $\mathrm{D}_{3 \mathrm{~h}}$ & 12 & 12 & 9 & 0 \\
\hline 149 & $\mathrm{C}_{18} \mathrm{H}_{18}$ & 2,4,5,7-tetramethylphenanthrene & $\mathrm{C}_{2}$ & 18 & 13 & 7 & 0 \\
\hline
\end{tabular}


Table S1, contd.

\begin{tabular}{|c|c|c|c|c|c|c|c|}
\hline MID & & Molecule & Point Group & ${ }^{3} \mathrm{C}-\mathrm{H}$ & ${ }^{3} \mathrm{C}-\mathrm{C}^{3}$ & ${ }^{2} \mathrm{C}=\mathrm{C}^{2}$ & ${ }^{1} \mathrm{C} \equiv \mathrm{C}^{1}$ \\
\hline 150 & $\mathrm{C}_{18} \mathrm{H}_{18}$ & 2,5-diphenyl-1,5-hexadiene & $\mathrm{C}_{2}$ & 18 & 11 & 8 & 0 \\
\hline 151 & $\mathrm{C}_{18} \mathrm{H}_{18}$ & 3,4,5,6-tetramethylphenanthrene & $\mathrm{C}_{2}$ & 18 & 13 & 7 & 0 \\
\hline 152 & $\mathrm{C}_{20}$ & $\mathrm{C}_{20}$ fullerene & $\mathrm{D}_{3 \mathrm{~d}}$ & 0 & 20 & 10 & 0 \\
\hline 153 & $\mathrm{C}_{20} \mathrm{H}_{10}$ & corannulene & $\mathrm{C}_{5 \mathrm{v}}$ & 10 & 15 & 10 & 0 \\
\hline 154 & $\mathrm{C}_{20} \mathrm{H}_{12}$ & perylene & $\mathrm{D}_{2}$ & 12 & 14 & 10 & 0 \\
\hline 155 & $\mathrm{C}_{20} \mathrm{H}_{14}$ & triptycene & $\mathrm{D}_{3 \mathrm{~h}}$ & 14 & 15 & 9 & 0 \\
\hline 156 & $\mathrm{C}_{20} \mathrm{H}_{20}$ & dodecahedrane & $\mathrm{I}_{\mathrm{h}}$ & 20 & 30 & 0 & 0 \\
\hline 157 & $\mathrm{C}_{20} \mathrm{H}_{20}$ & pagodane & $\mathrm{D}_{2 \mathrm{~h}}$ & 20 & 30 & 0 & 0 \\
\hline 158 & $\mathrm{C}_{20} \mathrm{H}_{36}$ & tetra-tert-butyltetrahedrane & $\mathrm{T}$ & 36 & 22 & 0 & 0 \\
\hline 159 & $\mathrm{C}_{24} \mathrm{H}_{12}$ & coronene & $\mathrm{D}_{3 \mathrm{~d}}$ & 12 & 18 & 12 & 0 \\
\hline 160 & $\mathrm{C}_{28} \mathrm{H}_{14}$ & {$[7]$ circulene $^{b}$} & $\mathrm{C}_{\mathrm{s}}$ & 14 & 21 & 14 & 0 \\
\hline 161 & $\mathrm{C}_{32} \mathrm{H}_{16}$ & [8]circulene & $\mathrm{D}_{2 \mathrm{~d}}$ & 16 & 24 & 16 & 0 \\
\hline
\end{tabular}


${ }^{a}$ There are two stationary points along the pseudorotation pathway of cyclopentane, one of $\mathrm{C}_{s}$ symmetry (envelope) and one of $\mathrm{C}_{2}$ symmetry (twisted). Geometry optimizations at both the MP2/cc-pVTZ and B3LYP/cc-pVTZ levels and subsequent analytical force constant calculations, performed with extremely tight convergence criteria for both the SCF and the geometry optimization, indicate that $\mathrm{C}_{2}$ is a minimum and $\mathrm{C}_{s}$ a transition state. The energy difference is about $0.001 \mathrm{kcal} / \mathrm{mol}(\mathrm{B} 3 \mathrm{LYP})$ or $0.002 \mathrm{kcal} / \mathrm{mol}$ (MP2), and single point calculations for MP2 geometries indicate equally negligible differences at HF, CCSD, and CCSD(T) levels with various basis sets (up to $0.003 \mathrm{kcal} / \mathrm{mol}$ ). Frequencies for all vibrational modes other than the lowest (pseudorotation) differ by $6 \mathrm{~cm}^{-1}$ at most and $1 \mathrm{~cm}^{-1}$ on average (signed average $<0.1 \mathrm{~cm}^{-1}$ ), both at the MP2 and B3LYP levels. For all intents and purposes the $\mathrm{C}_{2}$ and the $\mathrm{C}_{s}$ form should be considered isoenergetic, and the particular choice of conformer is irrelevant for thermochemical treatments, so long as the lowest-frequency mode is treated as having a small but real value ${ }^{89}$ (here taken to be $1 \mathrm{~cm}^{-1}$, see Sec. 3). All calculations reported have been performed for the $\mathrm{C}_{s}$ form.

${ }^{b}$ The optimized (RI-MP2/cc-pVTZ) geometry of [7]circulene shows one small imaginary frequency $\left(1.6 i \mathrm{~cm}^{-1}\right)$ which is very likely an artifact of the semi-analytical evaluation of force constants (see Sec. 3). Numerical proof by re-optimization at the regular (non-RI) MP2 level and fully analytical evaluation of force constants appeared to be prohibitively expensive and was not attempted. However, we found that also at the B3LYP/cc-pVTZ level only fully analytical frequency calculations afforded a real value $\left(2.9 \mathrm{~cm}^{-1} \mathrm{compared}\right.$ to $0.3 i \mathrm{~cm}^{-1}$ using semi-analytical second derivatives).

${ }^{c}$ Cyclopent[fg]acenaphthylene (131) is variably referred to as pyracylene or pyracyclene. We adopt the latter trivial name as suggested by Chemical Abstracts (SciFinder). 
Table S2: $\operatorname{CSSD}(T)$ atomization energies ${ }^{a}$

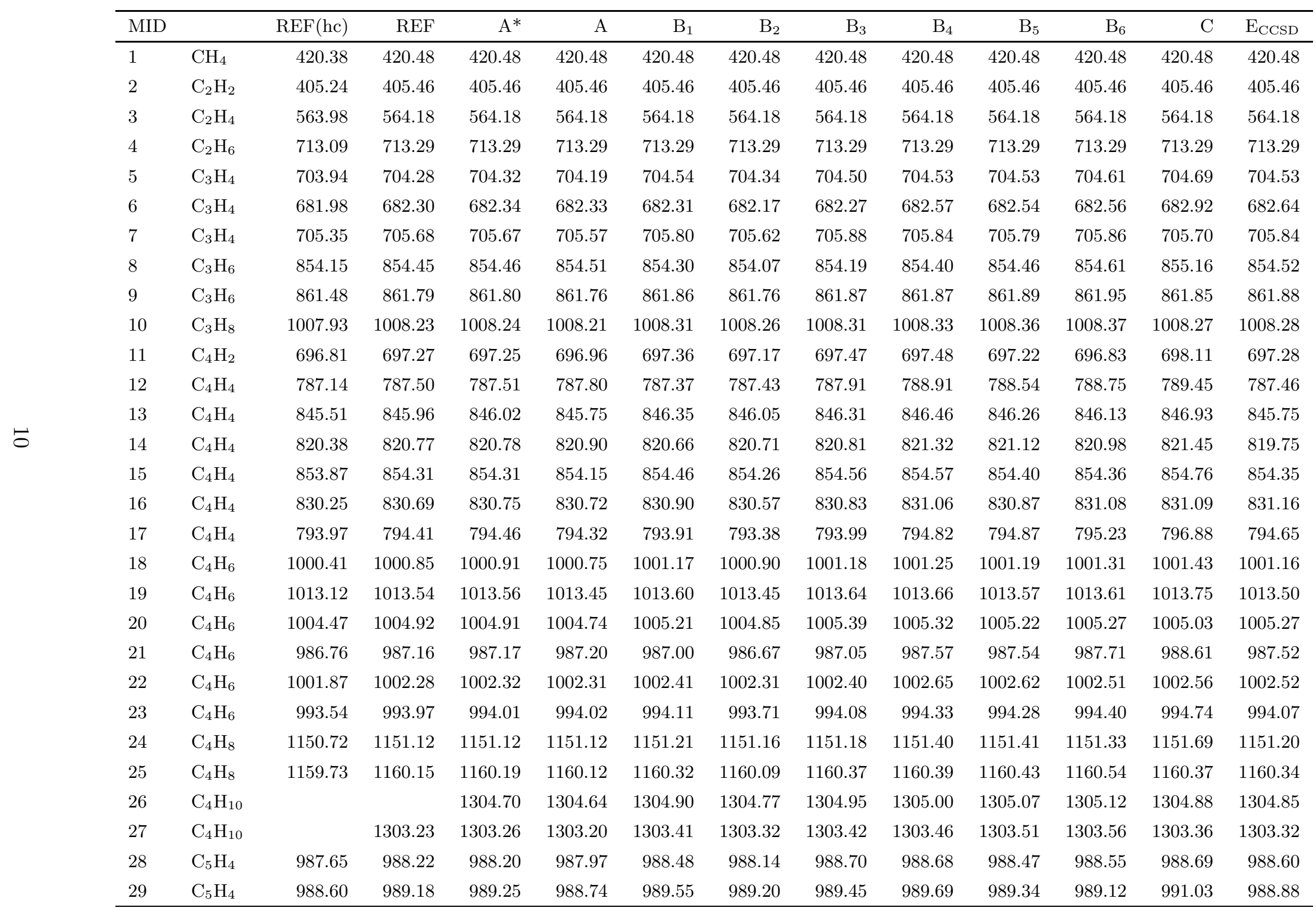


Table S2, contd.

\begin{tabular}{|c|c|c|c|c|c|c|c|c|c|c|c|c|c|}
\hline MID & & REF(hc) & REF & $\mathrm{A}^{*}$ & $\mathrm{~A}$ & $\mathrm{~B}_{1}$ & $\mathrm{~B}_{2}$ & $\mathrm{~B}_{3}$ & $\mathrm{~B}_{4}$ & $\mathrm{~B}_{5}$ & $\mathrm{~B}_{6}$ & $\mathrm{C}$ & $\mathrm{E}_{\mathrm{CCSD}}$ \\
\hline 30 & $\mathrm{C}_{5} \mathrm{H}_{4}$ & 941.72 & 942.25 & 942.32 & 942.37 & 942.46 & 942.11 & 942.41 & 943.03 & 942.70 & 942.74 & 943.38 & 942.50 \\
\hline 31 & $\mathrm{C}_{5} \mathrm{H}_{6}$ & & 1128.28 & 1128.28 & 1128.57 & 1128.03 & 1128.02 & 1128.53 & 1129.56 & 1129.20 & 1129.44 & 1131.18 & 1128.84 \\
\hline 32 & $\mathrm{C}_{5} \mathrm{H}_{6}$ & & 1182.77 & 1182.82 & 1182.76 & 1182.89 & 1182.49 & 1182.87 & 1183.02 & 1182.94 & 1182.98 & 1182.95 & 1183.21 \\
\hline 33 & $\mathrm{C}_{5} \mathrm{H}_{6}$ & & & 1154.36 & 1154.33 & 1154.51 & 1154.27 & 1154.58 & 1154.84 & 1154.64 & 1154.61 & 1154.94 & 1154.42 \\
\hline 34 & $\mathrm{C}_{5} \mathrm{H}_{6}$ & & & 1135.73 & 1135.79 & 1135.68 & 1135.39 & 1135.71 & 1136.31 & 1136.19 & 1136.09 & 1137.10 & 1135.97 \\
\hline 35 & $\mathrm{C}_{5} \mathrm{H}_{6}$ & & & 1143.23 & 1143.15 & 1143.30 & 1142.85 & 1143.35 & 1143.57 & 1143.46 & 1143.59 & 1144.28 & 1143.48 \\
\hline 36 & $\mathrm{C}_{5} \mathrm{H}_{8}$ & & & 1304.64 & 1304.58 & 1304.85 & 1304.64 & 1304.87 & 1304.92 & 1304.86 & 1304.95 & 1304.92 & 1304.83 \\
\hline 37 & $\mathrm{C}_{5} \mathrm{H}_{8}$ & & & 1311.66 & 1311.57 & 1311.77 & 1311.49 & 1311.85 & 1311.89 & 1311.83 & 1311.95 & 1312.23 & 1311.70 \\
\hline 38 & $\mathrm{C}_{5} \mathrm{H}_{8}$ & & 1286.51 & 1286.55 & 1286.64 & 1286.63 & 1285.99 & 1286.56 & 1287.03 & 1287.00 & 1287.11 & 1287.69 & 1286.69 \\
\hline 39 & $\mathrm{C}_{5} \mathrm{H}_{10}$ & & & 1466.29 & 1466.22 & 1466.60 & 1466.43 & 1466.54 & 1466.67 & 1466.74 & 1466.70 & 1466.68 & 1466.53 \\
\hline 40 & $\mathrm{C}_{5} \mathrm{H}_{12}$ & & & 1601.99 & 1601.91 & 1602.32 & 1602.06 & 1602.47 & 1602.59 & 1602.70 & 1602.74 & 1602.44 & 1602.26 \\
\hline 41 & $\mathrm{C}_{5} \mathrm{H}_{12}$ & & & 1598.23 & 1598.16 & 1598.49 & 1598.35 & 1598.51 & 1598.56 & 1598.62 & 1598.71 & 1598.43 & 1598.33 \\
\hline 42 & $\mathrm{C}_{6} \mathrm{H}_{2}$ & 989.52 & 990.18 & 990.13 & 989.49 & 990.09 & 989.82 & 990.37 & 990.39 & 989.81 & 989.02 & 992.47 & 990.13 \\
\hline 43 & $\mathrm{C}_{6} \mathrm{H}_{4}$ & 1101.41 & 1102.04 & 1102.11 & 1102.10 & 1102.03 & 1101.85 & 1102.39 & 1103.44 & 1102.79 & 1102.28 & 1104.52 & 1102.66 \\
\hline 44 & $\mathrm{C}_{6} \mathrm{H}_{4}$ & & & 1144.45 & 1144.15 & 1144.71 & 1144.29 & 1144.91 & 1144.95 & 1144.58 & 1144.51 & 1145.56 & 1144.67 \\
\hline 45 & $\mathrm{C}_{6} \mathrm{H}_{4}$ & 1132.07 & 1132.78 & 1132.87 & 1132.20 & 1133.10 & 1132.71 & 1133.02 & 1133.38 & 1132.80 & 1132.50 & 1135.48 & 1132.01 \\
\hline 46 & $\mathrm{C}_{6} \mathrm{H}_{4}$ & & 1162.77 & 1162.82 & 1162.82 & 1162.60 & 1162.04 & 1163.11 & 1163.69 & 1163.16 & 1162.73 & 1164.30 & 1162.44 \\
\hline 47 & $\mathrm{C}_{6} \mathrm{H}_{4}$ & & 1144.58 & 1144.60 & 1144.29 & 1144.90 & 1144.50 & 1145.09 & 1145.11 & 1144.72 & 1144.66 & 1145.57 & 1144.69 \\
\hline 48 & $\mathrm{C}_{6} \mathrm{H}_{4}$ & 1084.63 & 1085.29 & 1085.39 & 1085.30 & 1085.55 & 1084.92 & 1085.48 & 1086.17 & 1085.65 & 1085.90 & 1086.63 & 1085.76 \\
\hline 49 & $\mathrm{C}_{6} \mathrm{H}_{6}$ & & 1298.80 & 1298.74 & 1298.23 & 1298.85 & 1298.44 & 1299.13 & 1299.09 & 1298.69 & 1298.36 & 1300.43 & 1299.03 \\
\hline 50 & $\mathrm{C}_{6} \mathrm{H}_{6}$ & & 1246.29 & 1246.39 & 1246.34 & 1246.48 & 1246.05 & 1246.49 & 1247.12 & 1246.91 & 1247.04 & 1247.90 & 1247.03 \\
\hline 51 & $\mathrm{C}_{6} \mathrm{H}_{6}$ & & 1306.25 & 1306.31 & 1306.20 & 1306.47 & 1306.13 & 1306.57 & 1306.85 & 1306.48 & 1306.38 & 1307.15 & 1306.18 \\
\hline 52 & $\mathrm{C}_{6} \mathrm{H}_{6}$ & & 1280.87 & 1280.97 & 1280.87 & 1281.19 & 1280.61 & 1281.33 & 1281.68 & 1281.28 & 1280.94 & 1282.49 & 1280.38 \\
\hline 53 & $\mathrm{C}_{6} \mathrm{H}_{6}$ & 1369.59 & 1370.19 & 1370.28 & 1370.14 & 1370.40 & 1369.74 & 1370.25 & 1370.27 & 1369.93 & 1369.86 & 1370.50 & 1371.65 \\
\hline 54 & $\mathrm{C}_{6} \mathrm{H}_{6}$ & & 1295.86 & 1295.90 & 1295.93 & 1295.85 & 1295.30 & 1295.94 & 1296.68 & 1296.45 & 1296.49 & 1297.62 & 1296.46 \\
\hline 55 & $\mathrm{C}_{6} \mathrm{H}_{6}$ & & 1291.68 & 1291.77 & 1291.77 & 1291.94 & 1291.70 & 1291.93 & 1292.54 & 1292.26 & 1292.01 & 1292.83 & 1292.13 \\
\hline 56 & $\mathrm{C}_{6} \mathrm{H}_{6}$ & & 1336.96 & 1337.00 & 1336.89 & 1337.08 & 1336.60 & 1337.15 & 1337.32 & 1336.97 & 1336.98 & 1337.45 & 1337.26 \\
\hline 57 & $\mathrm{C}_{6} \mathrm{H}_{6}$ & & 1254.99 & 1255.01 & 1255.03 & 1254.84 & 1254.42 & 1254.87 & 1255.94 & 1255.79 & 1255.65 & 1257.41 & 1254.99 \\
\hline 58 & $\mathrm{C}_{6} \mathrm{H}_{8}$ & & & 1479.61 & 1479.54 & 1479.83 & 1479.37 & 1479.90 & 1480.02 & 1479.91 & 1480.03 & 1480.04 & 1479.79 \\
\hline 59 & $\mathrm{C}_{6} \mathrm{H}_{8}$ & & 1479.61 & 1479.68 & 1479.54 & 1479.96 & 1479.47 & 1480.02 & 1480.07 & 1480.01 & 1480.29 & 1480.18 & 1479.98 \\
\hline
\end{tabular}


Table S2, contd.

\begin{tabular}{|c|c|c|c|c|c|c|c|c|c|c|c|c|c|}
\hline MID & & REF(hc) & $\mathrm{REF}$ & $\mathrm{A}^{*}$ & A & $\mathrm{B}_{1}$ & $\mathrm{~B}_{2}$ & $\mathrm{~B}_{3}$ & $\mathrm{~B}_{4}$ & $\mathrm{~B}_{5}$ & $\mathrm{~B}_{6}$ & $\mathrm{C}$ & $\mathrm{E}_{\mathrm{CCSD}}$ \\
\hline 60 & $\mathrm{C}_{6} \mathrm{H}_{8}$ & & & 1463.67 & 1463.49 & 1463.79 & 1463.47 & 1463.86 & 1463.90 & 1463.70 & 1463.83 & 1464.19 & 1463.55 \\
\hline 61 & $\mathrm{C}_{6} \mathrm{H}_{10}$ & & & 1622.90 & 1622.79 & 1623.20 & 1622.83 & 1623.26 & 1623.35 & 1623.38 & 1623.52 & 1623.37 & 1623.17 \\
\hline 62 & $\mathrm{C}_{6} \mathrm{H}_{12}$ & & & 1767.68 & 1767.55 & 1768.03 & 1767.77 & 1768.00 & 1768.12 & 1768.23 & 1768.22 & 1767.98 & 1767.89 \\
\hline 63 & $\mathrm{C}_{6} \mathrm{H}_{14}$ & & & 1893.56 & 1893.46 & 1893.90 & 1893.68 & 1893.92 & 1894.02 & 1894.14 & 1894.26 & 1894.16 & 1893.64 \\
\hline 64 & $\mathrm{C}_{6} \mathrm{H}_{14}$ & & & 1893.25 & 1893.15 & 1893.58 & 1893.40 & 1893.61 & 1893.68 & 1893.76 & 1893.89 & 1893.54 & 1893.35 \\
\hline 65 & $\mathrm{C}_{7} \mathrm{H}_{4}$ & & 1257.93 & 1257.96 & 1257.70 & 1258.28 & 1257.71 & 1258.55 & 1258.89 & 1258.34 & 1258.46 & 1259.66 & 1258.59 \\
\hline 66 & $\mathrm{C}_{7} \mathrm{H}_{4}$ & & & 1277.08 & 1276.22 & 1277.34 & 1276.85 & 1277.21 & 1277.66 & 1276.90 & 1276.52 & 1280.37 & 1276.11 \\
\hline 67 & $\mathrm{C}_{7} \mathrm{H}_{6}$ & & & 1429.36 & 1429.18 & 1429.59 & 1428.93 & 1429.85 & 1430.12 & 1429.75 & 1429.86 & 1431.05 & 1429.85 \\
\hline 68 & $\mathrm{C}_{7} \mathrm{H}_{6}$ & & 1472.26 & 1472.36 & 1472.20 & 1472.49 & 1471.65 & 1472.56 & 1473.05 & 1472.50 & 1472.29 & 1474.02 & 1473.79 \\
\hline 69 & $\mathrm{C}_{7} \mathrm{H}_{6}$ & & 1476.99 & 1477.08 & 1476.83 & 1477.39 & 1476.74 & 1477.37 & 1477.61 & 1477.15 & 1477.32 & 1478.26 & 1477.69 \\
\hline 70 & $\mathrm{C}_{7} \mathrm{H}_{6}$ & & 1454.82 & 1454.92 & 1454.85 & 1455.05 & 1454.36 & 1455.06 & 1455.56 & 1455.13 & 1455.17 & 1455.98 & 1455.71 \\
\hline 71 & $\mathrm{C}_{7} \mathrm{H}_{8}$ & & & 1634.15 & 1634.08 & 1634.34 & 1633.74 & 1634.42 & 1634.60 & 1634.35 & 1634.42 & 1635.22 & 1634.76 \\
\hline 72 & $\mathrm{C}_{7} \mathrm{H}_{8}$ & & & 1621.67 & 1621.64 & 1621.91 & 1621.50 & 1622.01 & 1622.55 & 1622.34 & 1622.10 & 1622.88 & 1622.33 \\
\hline 73 & $\mathrm{C}_{7} \mathrm{H}_{8}$ & & & 1628.75 & 1628.74 & 1628.86 & 1628.08 & 1628.82 & 1629.21 & 1629.01 & 1629.03 & 1630.19 & 1629.18 \\
\hline 74 & $\mathrm{C}_{7} \mathrm{H}_{8}$ & & & 1599.31 & 1599.28 & 1599.35 & 1598.62 & 1599.39 & 1600.28 & 1600.18 & 1600.14 & 1601.45 & 1599.79 \\
\hline 75 & $\mathrm{C}_{7} \mathrm{H}_{8}$ & & & 1624.30 & 1624.31 & 1624.44 & 1623.68 & 1624.42 & 1624.88 & 1624.61 & 1624.62 & 1625.33 & 1624.94 \\
\hline 76 & $\mathrm{C}_{7} \mathrm{H}_{8}$ & & & 1667.36 & 1667.23 & 1667.59 & 1666.80 & 1667.53 & 1667.57 & 1667.23 & 1667.26 & 1668.03 & 1668.84 \\
\hline 77 & $\mathrm{C}_{7} \mathrm{H}_{12}$ & & & 1926.02 & 1925.91 & 1926.48 & 1926.13 & 1926.46 & 1926.78 & 1926.84 & 1926.72 & 1927.11 & 1926.54 \\
\hline 78 & $\mathrm{C}_{7} \mathrm{H}_{16}$ & & & 2188.27 & 2188.14 & 2188.69 & 2188.45 & 2188.72 & 2188.81 & 2188.90 & 2189.06 & 2188.65 & 2188.37 \\
\hline 79 & $\mathrm{C}_{8} \mathrm{H}_{2}$ & & & 1283.26 & 1282.30 & 1283.19 & 1282.79 & 1283.60 & 1283.67 & 1282.76 & 1281.53 & 1286.91 & 1283.33 \\
\hline 80 & $\mathrm{C}_{8} \mathrm{H}_{4}$ & & & 1418.70 & 1418.48 & 1419.05 & 1418.28 & 1419.43 & 1420.33 & 1419.39 & 1419.40 & 1421.73 & 1416.82 \\
\hline 81 & $\mathrm{C}_{8} \mathrm{H}_{4}$ & & & 1444.31 & 1444.13 & 1444.43 & 1443.67 & 1445.08 & 1445.58 & 1444.73 & 1444.27 & 1446.51 & 1444.13 \\
\hline 82 & $\mathrm{C}_{8} \mathrm{H}_{4}$ & & & 1421.38 & 1420.35 & 1421.61 & 1421.06 & 1421.48 & 1422.05 & 1421.06 & 1420.65 & 1425.48 & 1420.06 \\
\hline 83 & $\mathrm{C}_{8} \mathrm{H}_{6}$ & & & 1638.93 & 1638.85 & 1639.09 & 1638.37 & 1639.15 & 1639.58 & 1638.89 & 1638.69 & 1640.48 & 1639.55 \\
\hline 84 & $\mathrm{C}_{8} \mathrm{H}_{6}$ & & & 1576.94 & 1576.83 & 1577.07 & 1576.07 & 1577.34 & 1578.31 & 1577.54 & 1577.17 & 1580.01 & 1578.44 \\
\hline 85 & $\mathrm{C}_{8} \mathrm{H}_{6}$ & & & 1647.11 & 1647.04 & 1647.28 & 1646.54 & 1647.46 & 1647.86 & 1647.18 & 1647.18 & 1648.38 & 1647.26 \\
\hline 86 & $\mathrm{C}_{8} \mathrm{H}_{8}$ & & & 1780.89 & 1780.72 & 1781.16 & 1780.46 & 1781.31 & 1781.46 & 1781.07 & 1781.47 & 1782.45 & 1781.24 \\
\hline 87 & $\mathrm{C}_{8} \mathrm{H}_{8}$ & & & 1805.97 & 1805.83 & 1806.24 & 1805.42 & 1806.24 & 1806.50 & 1806.02 & 1805.93 & 1807.27 & 1807.45 \\
\hline 88 & $\mathrm{C}_{8} \mathrm{H}_{8}$ & & & 1707.40 & 1707.48 & 1707.31 & 1707.18 & 1707.37 & 1708.51 & 1708.29 & 1707.71 & 1710.19 & 1706.60 \\
\hline 89 & $\mathrm{C}_{8} \mathrm{H}_{8}$ & & & 1799.09 & 1798.91 & 1799.26 & 1798.51 & 1799.52 & 1799.65 & 1799.17 & 1799.43 & 1800.65 & 1798.71 \\
\hline
\end{tabular}


Table S2, contd.

\begin{tabular}{|c|c|c|c|c|c|c|c|c|c|c|c|c|c|}
\hline MID & & $\mathrm{REF}(\mathrm{hc})$ & REF & $\mathrm{A}^{*}$ & A & $\mathrm{B}_{1}$ & $\mathrm{~B}_{2}$ & $\mathrm{~B}_{3}$ & $\mathrm{~B}_{4}$ & $\mathrm{~B}_{5}$ & $\mathrm{~B}_{6}$ & $\mathrm{C}$ & $\mathrm{E}_{\mathrm{CCSD}}$ \\
\hline 90 & $\mathrm{C}_{8} \mathrm{H}_{8}$ & & & 1817.60 & 1817.39 & 1817.80 & 1816.98 & 1817.74 & 1817.79 & 1817.31 & 1817.37 & 1818.66 & 1818.96 \\
\hline 91 & $\mathrm{C}_{8} \mathrm{H}_{10}$ & & & 1914.06 & 1913.78 & 1914.23 & 1913.74 & 1914.34 & 1914.40 & 1914.08 & 1914.30 & 1914.89 & 1913.87 \\
\hline 92 & $\mathrm{C}_{8} \mathrm{H}_{14}$ & & & 2225.93 & 2225.76 & 2226.47 & 2226.05 & 2226.47 & 2226.75 & 2226.85 & 2226.81 & 2227.13 & 2226.35 \\
\hline 93 & $\mathrm{C}_{8} \mathrm{H}_{18}$ & & & 2483.29 & 2483.14 & 2483.79 & 2483.51 & 2483.83 & 2483.93 & 2484.04 & 2484.24 & 2483.77 & 2483.40 \\
\hline 94 & $\mathrm{C}_{9} \mathrm{H}_{20}$ & & & 2778.31 & 2778.13 & 2778.90 & 2778.57 & 2778.94 & 2779.06 & 2779.18 & 2779.42 & 2778.89 & 2778.42 \\
\hline 95 & $\mathrm{C}_{10} \mathrm{H}_{2}$ & & & 1576.41 & 1575.15 & 1576.26 & 1575.73 & 1576.82 & 1576.94 & 1575.70 & 1574.00 & 1581.41 & 1576.53 \\
\hline 96 & $\mathrm{C}_{10} \mathrm{H}_{4}$ & & & 1719.57 & 1718.92 & 1719.81 & 1719.07 & 1720.38 & 1720.57 & 1719.63 & 1719.69 & 1723.06 & 1720.34 \\
\hline 97 & $\mathrm{C}_{10} \mathrm{H}_{8}$ & & & 2130.26 & 2129.98 & 2130.39 & 2129.31 & 2130.62 & 2130.77 & 2129.94 & 2130.37 & 2132.52 & 2131.64 \\
\hline 98 & $\mathrm{C}_{10} \mathrm{H}_{8}$ & & & 2166.97 & 2166.71 & 2167.14 & 2165.87 & 2167.10 & 2167.15 & 2166.36 & 2166.49 & 2168.66 & 2169.08 \\
\hline 99 & $\mathrm{C}_{10} \mathrm{H}_{8}$ & & & 2111.16 & 2110.98 & 2111.33 & 2110.37 & 2111.45 & 2111.77 & 2110.97 & 2111.25 & 2112.67 & 2111.78 \\
\hline 100 & $\mathrm{C}_{10} \mathrm{H}_{10}$ & & & 2174.85 & 2174.40 & 2174.79 & 2173.83 & 2175.00 & 2175.49 & 2174.91 & 2174.71 & 2178.67 & 2175.22 \\
\hline 101 & $\mathrm{C}_{10} \mathrm{H}_{10}$ & & & 2254.93 & 2254.61 & 2255.26 & 2254.27 & 2255.39 & 2255.43 & 2254.73 & 2254.79 & 2256.86 & 2256.57 \\
\hline 102 & $\mathrm{C}_{10} \mathrm{H}_{10}$ & & & & 2245.68 & 2246.23 & 2244.98 & 2246.21 & 2246.56 & 2246.02 & 2246.10 & 2247.93 & 2247.37 \\
\hline 103 & $\mathrm{C}_{10} \mathrm{H}_{10}$ & & & 2229.18 & 2229.04 & 2229.59 & 2228.66 & 2229.90 & 2230.31 & 2229.66 & 2229.91 & 2231.88 & 2228.88 \\
\hline 104 & $\mathrm{C}_{10} \mathrm{H}_{10}$ & & & 2208.03 & 2208.01 & 2208.45 & 2207.86 & 2208.60 & 2209.59 & 2209.23 & 2208.84 & 2211.24 & 2208.19 \\
\hline 105 & $\mathrm{C}_{10} \mathrm{H}_{10}$ & & & 2243.49 & 2243.36 & 2243.92 & 2242.95 & 2244.06 & 2244.58 & 2243.95 & 2243.83 & 2245.91 & 2245.09 \\
\hline 106 & $\mathrm{C}_{10} \mathrm{H}_{10}$ & & & 2235.99 & 2235.96 & 2236.49 & 2235.34 & 2236.70 & 2237.26 & 2236.86 & 2237.06 & 2238.65 & 2236.64 \\
\hline 107 & $\mathrm{C}_{10} \mathrm{H}_{10}$ & & & 2227.48 & 2227.58 & 2227.82 & 2226.48 & 2228.00 & 2229.16 & 2228.88 & 2228.93 & 2230.93 & 2228.18 \\
\hline 108 & $\mathrm{C}_{10} \mathrm{H}_{10}$ & & & 2193.64 & 2193.03 & 2193.84 & 2193.15 & 2194.33 & 2194.45 & 2193.75 & 2193.46 & 2196.96 & 2193.98 \\
\hline 109 & $\mathrm{C}_{10} \mathrm{H}_{10}$ & & & 2227.77 & 2227.67 & 2228.23 & 2227.46 & 2228.45 & 2229.20 & 2228.81 & 2228.68 & 2230.25 & 2228.86 \\
\hline 110 & $\mathrm{C}_{10} \mathrm{H}_{10}$ & & & 2244.26 & 2244.25 & 2244.58 & 2243.37 & 2244.82 & 2245.79 & 2245.45 & 2245.45 & 2247.23 & 2245.19 \\
\hline 111 & $\mathrm{C}_{10} \mathrm{H}_{10}$ & & & 2259.08 & 2258.97 & 2259.83 & 2258.98 & 2260.11 & 2260.55 & 2260.12 & 2260.36 & 2261.02 & 2259.86 \\
\hline 112 & $\mathrm{C}_{10} \mathrm{H}_{12}$ & & & 2364.53 & 2364.16 & 2364.76 & 2364.09 & 2364.90 & 2364.98 & 2364.54 & 2364.86 & 2365.70 & 2364.28 \\
\hline 113 & $\mathrm{C}_{10} \mathrm{H}_{16}$ & & & 2700.35 & 2700.08 & 2701.22 & 2700.51 & 2701.33 & 2701.69 & 2701.76 & 2701.71 & 2702.06 & 2701.05 \\
\hline 114 & $\mathrm{C}_{10} \mathrm{H}_{16}$ & & & & 2690.19 & 2691.26 & 2690.64 & 2691.32 & 2691.72 & 2691.77 & 2691.78 & 2692.39 & 2690.93 \\
\hline 115 & $\mathrm{C}_{10} \mathrm{H}_{18}$ & & & & 2823.67 & 2824.85 & 2824.27 & 2824.89 & 2825.17 & 2825.32 & 2825.43 & 2825.37 & 2824.40 \\
\hline 116 & $\mathrm{C}_{10} \mathrm{H}_{18}$ & & & 2826.62 & 2826.39 & 2827.47 & 2826.85 & 2827.54 & 2827.81 & 2827.95 & 2828.13 & 2827.87 & 2827.04 \\
\hline 117 & $\mathrm{C}_{10} \mathrm{H}_{22}$ & & & & 3073.07 & 3074.00 & 3073.63 & 3074.04 & 3074.18 & 3074.32 & 3074.60 & 3074.01 & 3073.45 \\
\hline 118 & $\mathrm{C}_{11} \mathrm{H}_{24}$ & & & & 3368.06 & 3369.11 & 3368.70 & 3369.15 & 3369.31 & 3369.46 & 3369.78 & 3369.13 & 3368.48 \\
\hline 119 & $\mathrm{C}_{12} \mathrm{H}_{6}$ & & & 2175.60 & 2175.64 & 2175.52 & 2174.37 & 2176.48 & 2178.23 & 2176.70 & 2176.12 & 2180.44 & 2174.88 \\
\hline
\end{tabular}


Table S2, contd.

\begin{tabular}{|c|c|c|c|c|c|c|c|c|c|c|c|c|c|}
\hline MID & & REF(hc) & REF & $\mathrm{A}^{*}$ & A & $\mathrm{B}_{1}$ & $\mathrm{~B}_{2}$ & $\mathrm{~B}_{3}$ & $\mathrm{~B}_{4}$ & $\mathrm{~B}_{5}$ & $\mathrm{~B}_{6}$ & $\mathrm{C}$ & $\mathrm{E}_{\mathrm{CCSD}}$ \\
\hline 120 & $\mathrm{C}_{12} \mathrm{H}_{6}$ & & & 2072.64 & 2072.43 & 2073.11 & 2071.60 & 2073.02 & 2074.37 & 2072.92 & 2073.27 & 2076.43 & 2073.39 \\
\hline 121 & $\mathrm{C}_{12} \mathrm{H}_{8}$ & & & 2487.75 & 2487.44 & 2487.96 & 2486.38 & 2488.07 & 2488.28 & 2487.11 & 2487.25 & 2490.45 & 2490.12 \\
\hline 122 & $\mathrm{C}_{12} \mathrm{H}_{8}$ & & & 2450.09 & 2449.77 & 2450.29 & 2448.93 & 2450.30 & 2450.71 & 2449.52 & 2449.30 & 2453.00 & 2452.35 \\
\hline 123 & $\mathrm{C}_{12} \mathrm{H}_{10}$ & & & 2623.53 & 2623.26 & 2623.94 & 2622.44 & 2623.80 & 2623.89 & 2622.98 & 2623.07 & 2625.43 & 2626.41 \\
\hline 124 & $\mathrm{C}_{12} \mathrm{H}_{12}$ & & & & & & & 2728.00 & 2728.23 & 2727.56 & 2727.92 & 2729.98 & 2729.14 \\
\hline 125 & $\mathrm{C}_{12} \mathrm{H}_{14}$ & & & 2815.03 & 2814.56 & 2815.31 & 2814.47 & 2815.50 & 2815.60 & 2815.03 & 2815.45 & 2816.53 & 2814.72 \\
\hline 126 & $\mathrm{C}_{12} \mathrm{H}_{18}$ & & & & 3145.39 & 3146.02 & 3144.67 & 3146.25 & 3146.55 & 3146.30 & 3146.79 & 3148.75 & 3147.15 \\
\hline 127 & $\mathrm{C}_{12} \mathrm{H}_{26}$ & & & & 3663.05 & 3664.22 & 3663.76 & 3664.26 & 3664.44 & 3664.60 & 3664.96 & 3664.25 & 3663.51 \\
\hline 128 & $\mathrm{C}_{13} \mathrm{H}_{10}$ & & & & 2795.49 & 2796.21 & 2794.50 & 2796.22 & 2796.39 & 2795.34 & 2795.55 & 2798.31 & 2798.89 \\
\hline 129 & $\mathrm{C}_{13} \mathrm{H}_{28}$ & & & & 3958.04 & 3959.32 & 3958.82 & 3959.37 & 3959.57 & 3959.74 & 3960.14 & 3959.37 & 3958.53 \\
\hline 130 & $\mathrm{C}_{14} \mathrm{H}_{8}$ & & & & 2760.54 & 2760.73 & 2758.98 & 2761.33 & 2761.94 & 2760.38 & 2760.34 & 2765.25 & 2762.70 \\
\hline 131 & $\mathrm{C}_{14} \mathrm{H}_{8}$ & & & 2795.03 & 2794.70 & 2795.18 & 2793.37 & 2795.48 & 2795.93 & 2794.36 & 2794.44 & 2799.11 & 2797.15 \\
\hline 132 & $\mathrm{C}_{14} \mathrm{H}_{10}$ & & & 2959.90 & 2959.50 & 2960.10 & 2958.23 & 2960.19 & 2960.32 & 2959.06 & 2959.46 & 2963.19 & 2962.51 \\
\hline 133 & $\mathrm{C}_{14} \mathrm{H}_{10}$ & & & & 2965.56 & 2966.18 & 2964.32 & 2966.15 & 2966.27 & 2964.97 & 2965.30 & 2969.27 & 2969.04 \\
\hline 134 & $\mathrm{C}_{14} \mathrm{H}_{20}$ & & & 3635.53 & 3635.21 & 3637.14 & 3635.92 & 3637.43 & 3638.02 & 3637.98 & 3638.00 & 3638.86 & 3636.81 \\
\hline 135 & $\mathrm{C}_{14} \mathrm{H}_{30}$ & & & & 4253.03 & 4254.43 & 4253.88 & 4254.48 & 4254.70 & 4254.89 & 4255.32 & 4254.49 & 4253.56 \\
\hline 136 & $\mathrm{C}_{15} \mathrm{H}_{32}$ & & & & 4548.02 & 4549.54 & 4548.94 & 4549.59 & 4549.82 & 4550.03 & 4550.50 & 4549.61 & 4548.59 \\
\hline 137 & $\mathrm{C}_{16} \mathrm{H}_{8}$ & & & 3050.64 & 3050.47 & 3051.10 & 3049.09 & 3051.71 & 3052.95 & 3050.92 & 3050.43 & 3055.68 & 3052.61 \\
\hline 138 & $\mathrm{C}_{16} \mathrm{H}_{10}$ & & & & 3294.67 & 3295.47 & 3293.29 & 3295.59 & 3295.82 & 3294.15 & 3294.41 & 3299.34 & 3298.92 \\
\hline 139 & $\mathrm{C}_{16} \mathrm{H}_{10}$ & & & 3309.73 & 3309.24 & 3309.81 & 3307.56 & 3309.95 & 3310.10 & 3308.45 & 3308.95 & 3314.33 & 3313.07 \\
\hline 140 & $\mathrm{C}_{16} \mathrm{H}_{12}$ & & & & 3353.19 & 3353.94 & 3352.00 & 3354.56 & 3355.38 & 3354.11 & 3354.52 & 3358.89 & 3356.97 \\
\hline 141 & $\mathrm{C}_{16} \mathrm{H}_{16}$ & & & & 3669.56 & 3670.70 & 3668.84 & 3671.05 & 3671.61 & 3670.78 & 3670.87 & 3674.54 & 3673.75 \\
\hline 142 & $\mathrm{C}_{16} \mathrm{H}_{16}$ & & & & 3661.52 & 3662.49 & 3660.64 & 3663.04 & 3663.72 & 3662.89 & 3663.09 & 3667.46 & 3665.95 \\
\hline 143 & $\mathrm{C}_{16} \mathrm{H}_{16}$ & & & & 3650.24 & 3651.19 & 3649.36 & 3651.68 & 3652.42 & 3651.58 & 3651.77 & 3656.04 & 3654.32 \\
\hline 144 & $\mathrm{C}_{16} \mathrm{H}_{34}$ & & & & 4843.02 & 4844.65 & 4844.00 & 4844.70 & 4844.95 & 4845.17 & 4845.68 & 4844.73 & 4843.62 \\
\hline 145 & $\mathrm{C}_{18} \mathrm{H}_{12}$ & & & & 3757.30 & 3758.25 & 3755.77 & 3758.30 & 3758.53 & 3756.74 & 3757.21 & 3762.84 & 3761.84 \\
\hline 146 & $\mathrm{C}_{18} \mathrm{H}_{12}$ & & & & 3762.94 & 3763.69 & 3761.26 & 3763.70 & 3763.88 & 3762.07 & 3762.62 & 3768.49 & 3767.41 \\
\hline 147 & $\mathrm{C}_{18} \mathrm{H}_{12}$ & & & & 3750.57 & 3751.36 & 3748.89 & 3751.61 & 3751.84 & 3750.09 & 3750.83 & 3756.16 & 3754.14 \\
\hline 148 & $\mathrm{C}_{18} \mathrm{H}_{12}$ & & & & 3763.53 & 3764.22 & 3761.83 & 3764.16 & 3764.36 & 3762.49 & 3763.04 & 3769.21 & 3768.14 \\
\hline 149 & $\mathrm{C}_{18} \mathrm{H}_{18}$ & & & & 4141.53 & 4142.69 & 4140.33 & 4143.04 & 4143.42 & 4142.14 & 4142.90 & 4147.50 & 4145.43 \\
\hline
\end{tabular}


Table S2, contd.

\begin{tabular}{|c|c|c|c|c|c|c|c|c|c|c|c|c|c|}
\hline MID & & $\mathrm{REF}(\mathrm{hc})$ & $\mathrm{REF}$ & $\mathrm{A}^{*}$ & $\mathrm{~A}$ & $\mathrm{~B}_{1}$ & $\mathrm{~B}_{2}$ & $\mathrm{~B}_{3}$ & $\mathrm{~B}_{4}$ & $\mathrm{~B}_{5}$ & $\mathrm{~B}_{6}$ & $\mathrm{C}$ & $\mathrm{E}_{\mathrm{CCSD}}$ \\
\hline 150 & $\mathrm{C}_{18} \mathrm{H}_{18}$ & & & & 4107.16 & 4108.39 & 4106.42 & 4108.42 & 4108.73 & 4107.71 & 4108.10 & 4111.83 & 4110.89 \\
\hline 151 & $\mathrm{C}_{18} \mathrm{H}_{18}$ & & & & 4140.78 & 4141.83 & 4139.48 & 4142.10 & 4142.52 & 4141.31 & 4142.03 & 4146.72 & 4144.62 \\
\hline 152 & $\mathrm{C}_{20}$ & & & 2916.30 & 2916.45 & 2914.82 & 2912.58 & 2918.19 & 2923.07 & 2918.74 & 2917.32 & 2926.07 & 2917.92 \\
\hline 153 & $\mathrm{C}_{20} \mathrm{H}_{10}$ & & & & 3944.22 & 3945.57 & 3942.53 & 3945.98 & 3946.55 & 3944.06 & 3944.25 & 3950.82 & 3949.41 \\
\hline 154 & $\mathrm{C}_{20} \mathrm{H}_{12}$ & & & & 4100.26 & 4100.99 & 4098.20 & 4101.17 & 4101.46 & 4099.25 & 4100.02 & 4107.48 & 4104.93 \\
\hline 155 & $\mathrm{C}_{20} \mathrm{H}_{14}$ & & & & 4213.33 & 4214.55 & 4211.71 & 4214.79 & 4215.37 & 4213.56 & 4213.45 & 4218.43 & 4218.91 \\
\hline 156 & $\mathrm{C}_{20} \mathrm{H}_{20}$ & & & & 4621.13 & 4624.48 & 4622.58 & 4624.97 & 4626.00 & 4625.29 & 4625.47 & 4626.55 & 4622.31 \\
\hline 157 & $\mathrm{C}_{20} \mathrm{H}_{20}$ & & & & 4589.83 & 4591.76 & 4589.77 & 4592.88 & 4594.61 & 4593.67 & 4593.13 & 4598.66 & 4592.02 \\
\hline 158 & $\mathrm{C}_{20} \mathrm{H}_{36}$ & & & & & & & 5548.59 & 5550.25 & 5550.07 & 5550.28 & 5555.79 & 5546.90 \\
\hline 159 & $\mathrm{C}_{24} \mathrm{H}_{12}$ & & & & 4803.12 & 4803.84 & 4800.15 & 4804.17 & 4804.44 & 4801.49 & 4802.42 & 4812.50 & 4809.46 \\
\hline 160 & $\mathrm{C}_{28} \mathrm{H}_{14}$ & & & & & & & 5551.49 & 5552.18 & 5548.66 & 5549.95 & 5561.34 & 5556.73 \\
\hline 161 & $\mathrm{C}_{32} \mathrm{H}_{16}$ & & & & & & & 6305.20 & 6306.34 & 6302.31 & 6303.88 & 6316.52 & 6310.70 \\
\hline
\end{tabular}

${ }^{a}$ All values are given in kcal/mol. $\mathrm{REF}(\mathrm{hc})$ is the $\mathrm{ATOMIC}(\mathrm{hc})$ reference $\left(E_{A, e}^{\mathrm{CCSD}(\mathrm{T})(H)}[M]+C_{A, e}^{\mathrm{CCSD}(\mathrm{T})(H)}[M]\right)$, which reads $[\overline{\mathbf{6}} \overline{\mathbf{6}} \overline{\mathbf{6}} \overline{\mathbf{6}} \mid \overline{\mathbf{6}}$ $\overline{\mathbf{6}} \overline{\mathbf{6}} \overline{\mathbf{5}}]$ in ATOMIC notation and uses an updated CBS extrapolation procedure (See Ref. 38), REF is the standard ATOMIC reference level $\left(E_{A, e}^{\mathrm{CCSD}(\mathrm{T})(H)}[M]\right)$, which reads $[\overline{\mathbf{6}} \overline{\mathbf{6}} \overline{\mathbf{6}} \overline{\mathbf{5}} \mid \overline{\mathbf{5}} \overline{\mathbf{5}} \overline{\mathbf{5}} \overline{\mathbf{5}}]$. Values for models $(m) \mathrm{A}^{*}$ to $\mathrm{E}_{\mathrm{CCSD}}$ are reported as ATOMIC/m results $\left(E_{A, e}^{\mathrm{CCSD}(\mathrm{T})(m)}[M]\right)$, i.e. with BSR corrections toward the standard ATOMIC reference (REF). Molecules are identified by their molecule index MID as specified in Table S1. 


$\mathrm{CH}_{4}$
$\mathrm{CH}$
1.085
0.003
0.002
0.001

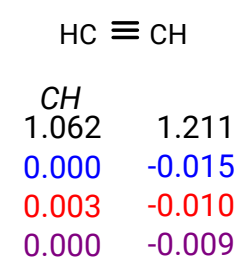

acetylene

$\mathrm{H}_{2} \mathrm{C}=\mathrm{C}=\mathrm{CH}_{2}$
1.308
-0.008
-0.002
-0.002
allene
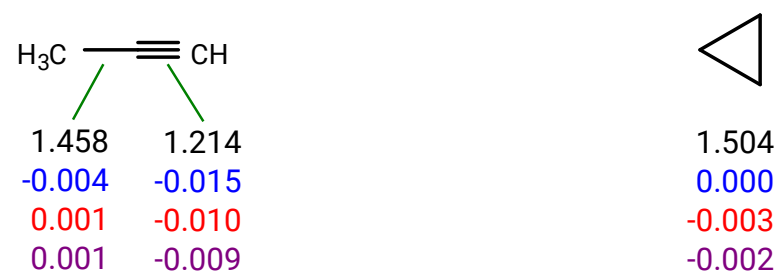

propyne
$\mathrm{H}_{3} \mathrm{C}-\mathrm{CH}_{3}$

$1.088 \quad 1.523$

$0.003 \quad 0.004$

$0.002-0.002$

$0.001-0.001$

ethane

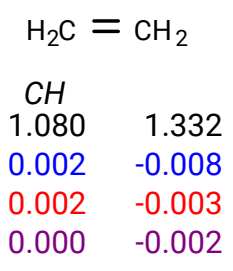

ethylene
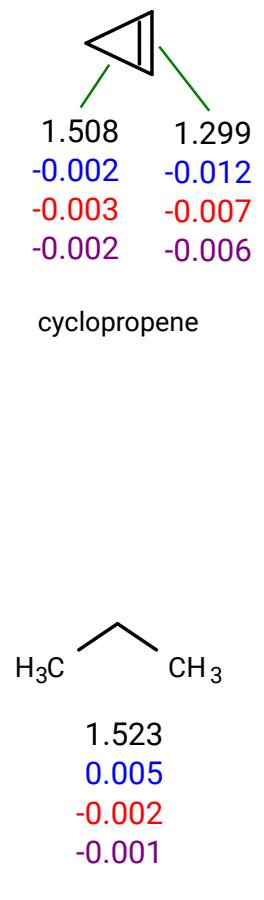

propane

Shown are CC bond lengths in $\AA$ at the RI-MP2/cc-pVTZ (top, black), B3LYP/cc-pVTZ (second, blue), model B6 (third, red), and model A (fourth, purple) levels. The latter three are expressed as difference with respect to the first. $\mathrm{CH}$ bond lengths are reported only for the four BSR prototype molecules.

Figure S1: Key geometric parameters, part 1 


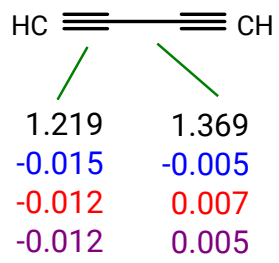

1,3-butadiyne
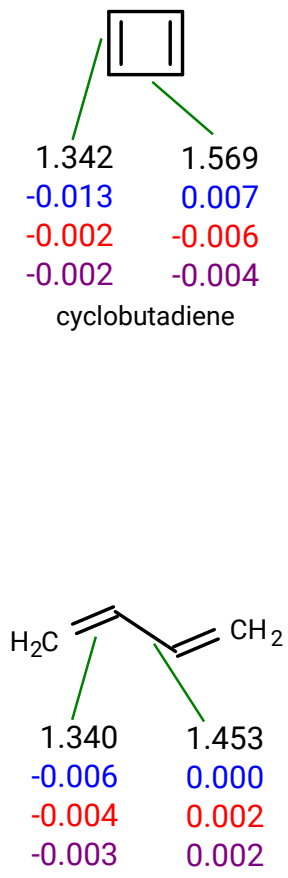

1,3-butadiene

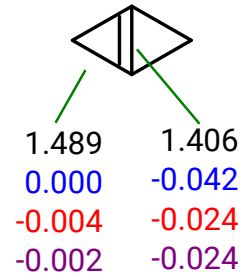

bicyclo[1.1.0]but-1(3)-ene
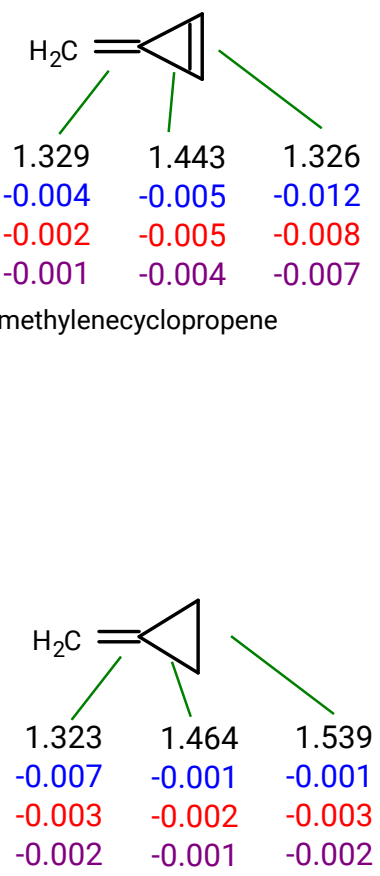

methylenecyclopropane

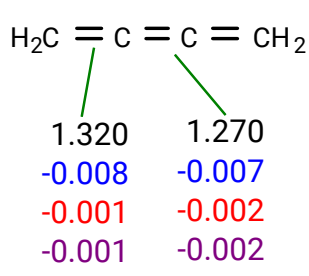

butatriene

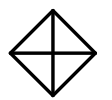

1.478

$-0.004$

$-0.004$

$-0.003$

tetrahedrane

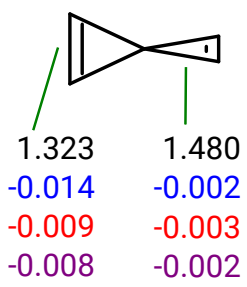

spiropentadiene

Shown are CC bond lengths in $\AA$ at the RI-MP2/cc-pVTZ (top, black), B3LYP/cc-pVTZ (second, blue), model B6 (third, red), and model A (fourth, purple) levels. The latter three are expressed as difference with respect to the first.

Figure S2: Key geometric parameters, part 2 

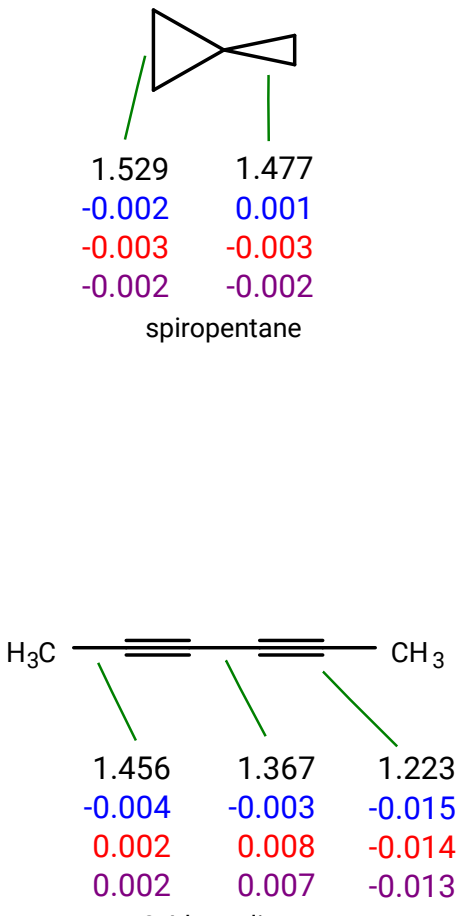

2,4-hexadiyne

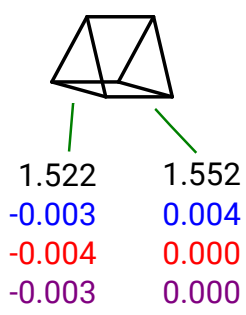

prismane
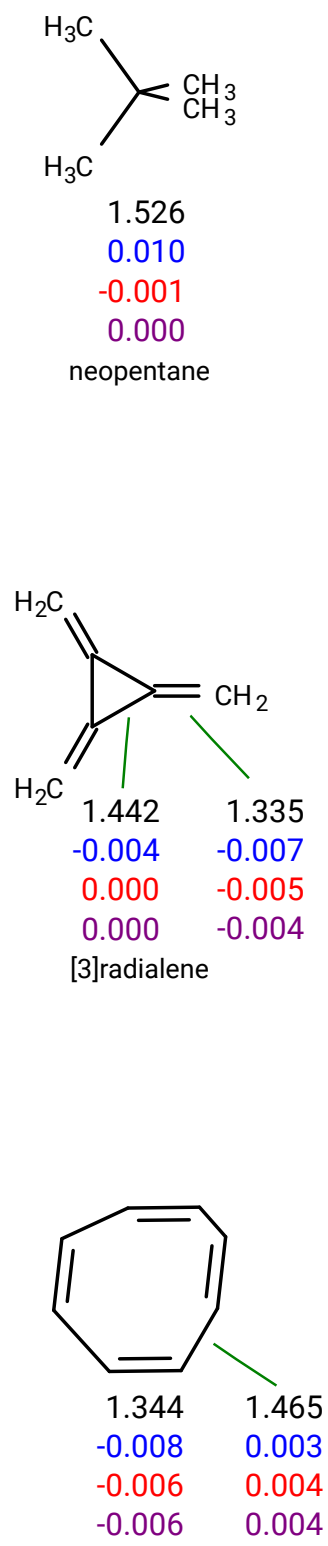

1,3,5,7-cyclooctatetraene
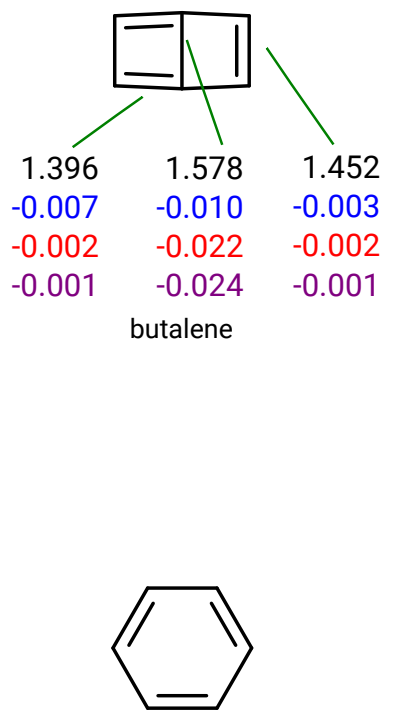

1.394

$-0.003$

$-0.003$

$-0.003$

benzene

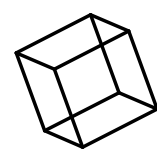

1.566

0.001

$-0.002$

cubane

Shown are CC bond lengths in $\AA$ at the RI-MP2/cc-pVTZ (top, black), B3LYP/cc-pVTZ (second, blue), model B6 (third, red), and, if available, model A (fourth, purple) levels. The latter three are expressed as difference with respect to the first.

Figure S3: Key geometric parameters, part 3 


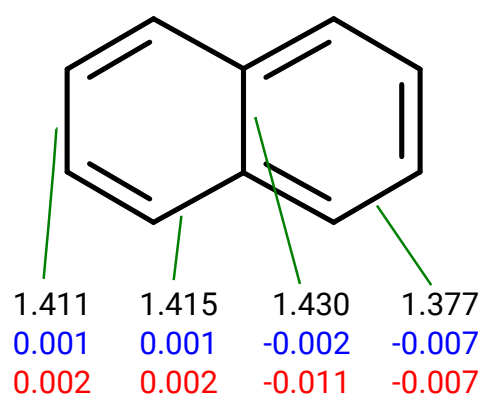

naphthalene

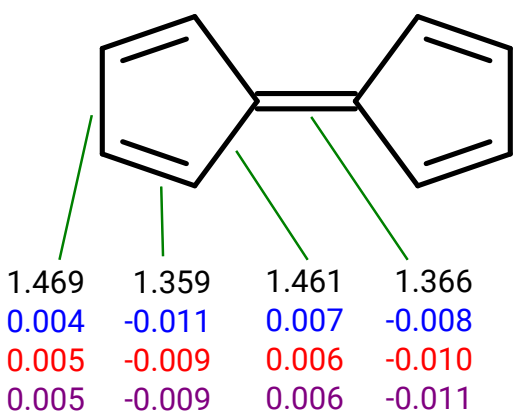

pentafulvalene

Shown are CC bond lengths in $\AA$ at the RI-MP2/cc-pVTZ (top, black), B3LYP/cc-pVTZ (second, blue), model B6 (third, red), and, if available, model A (fourth, purple) levels. The latter three are expressed as difference with respect to the first.

Figure S4: Key geometric parameters, part 4 
Table S3: Bond lengths $(\AA)$ : Comparison to accurate literature data ${ }^{a}$

\begin{tabular}{|c|c|c|c|c|c|c|c|c|c|}
\hline \multirow[b]{2}{*}{$\mathrm{CH}_{4}$} & \multirow[b]{2}{*}{ methane } & \multirow[b]{2}{*}{$\mathrm{C}-\mathrm{H}$} & \multirow{2}{*}{$\begin{array}{l}\text { model A } \\
1.0860\end{array}$} & \multicolumn{3}{|c|}{ literature value / referene } & \multicolumn{3}{|c|}{ literature value / reference } \\
\hline & & & & $1.0862(5)$ & $\mathrm{E}$ & 95 & $1.0859(3)$ & $\mathrm{T}$ & 96 \\
\hline \multirow[t]{2}{*}{$\mathrm{C}_{2} \mathrm{H}_{2}$} & acetylene & $\mathrm{C}-\mathrm{H}$ & 1.0618 & $1.06164(1)$ & $\mathrm{E}$ & 97 & & & \\
\hline & & $\mathrm{C} \equiv \mathrm{C}$ & 1.2024 & $1.20296(1)$ & $\mathrm{E}$ & 97 & & & \\
\hline \multirow[t]{2}{*}{$\mathrm{C}_{2} \mathrm{H}_{4}$} & ethylene & $\mathrm{C}-\mathrm{H}$ & 1.0808 & $1.0805(10)$ & $\mathrm{E}$ & 98 & 1.0804 & $\mathrm{~T}$ & 99 \\
\hline & & $\mathrm{C}=\mathrm{C}$ & 1.3301 & $1.3305(10)$ & $\mathrm{E}$ & 98 & 1.3305 & $\mathrm{~T}$ & 99 \\
\hline \multirow[t]{2}{*}{$\mathrm{C}_{2} \mathrm{H}_{6}$} & ethane & $\mathrm{C}-\mathrm{H}$ & 1.0890 & 1.0895 & $\mathrm{~T}$ & 100 & & & \\
\hline & & $\mathrm{C}-\mathrm{C}$ & 1.5224 & 1.5240 & $\mathrm{~T}$ & 100 & 1.5219 & $\mathrm{~T}$ & 101 \\
\hline \multirow[t]{2}{*}{$\mathrm{C}_{3} \mathrm{H}_{4}$} & allene & $\mathrm{C}-\mathrm{H}$ & 1.0809 & $1.081(2)$ & $\mathrm{E}$ & 102 & 1.0807 & $\mathrm{~T}$ & 103 \\
\hline & & $\mathrm{C}=\mathrm{C}$ & 1.3066 & $1.307(1)$ & $\mathrm{E}$ & 102 & 1.3063 & $\mathrm{~T}$ & 103 \\
\hline \multirow[t]{4}{*}{$\mathrm{C}_{3} \mathrm{H}_{4}$} & cyclopropene & $-\mathrm{C}-\mathrm{H}$ & 1.0852 & $1.0850(3)$ & $\mathrm{E}$ & 104 & & & \\
\hline & & $=\mathrm{C}-\mathrm{H}$ & 1.0730 & $1.0723(2)$ & $\mathrm{E}$ & 104 & & & \\
\hline & & $\mathrm{C}-\mathrm{C}$ & 1.5060 & $1.5066(1)$ & $\mathrm{E}$ & 104 & & & \\
\hline & & $\mathrm{C}=\mathrm{C}$ & 1.2928 & 1.2937 & $\mathrm{E}$ & 104 & & & \\
\hline \multirow[t]{2}{*}{$\mathrm{C}_{3} \mathrm{H}_{6}$} & cyclopropane & $\mathrm{C}-\mathrm{H}$ & 1.0789 & $1.0786(10)$ & $\mathrm{E}$ & 105 & 1.0791 & $\mathrm{~T}$ & 99 \\
\hline & & $\mathrm{C}-\mathrm{C}$ & 1.5022 & $1.5030(10)$ & $\mathrm{E}$ & 105 & 1.5024 & $\mathrm{~T}$ & 99 \\
\hline \multirow[t]{3}{*}{$\mathrm{C}_{4} \mathrm{H}_{2}$} & 1,3-butadiyne & $\mathrm{C}-\mathrm{H}$ & 1.0617 & 1.0615 & $\mathrm{E}$ & 106 & 1.0617 & $\mathrm{~T}$ & 106 \\
\hline & & $\mathrm{C}-\mathrm{C}$ & 1.3738 & 1.3727 & $\mathrm{E}$ & 106 & 1.3737 & $\mathrm{~T}$ & 106 \\
\hline & & $\mathrm{C} \equiv \mathrm{C}$ & 1.2079 & 1.2085 & $\mathrm{E}$ & 106 & 1.2083 & $\mathrm{~T}$ & 106 \\
\hline \multirow[t]{5}{*}{$\mathrm{C}_{4} \mathrm{H}_{6}$} & 1,3-butadiene & $-\mathrm{C}-\mathrm{H}$ & 1.0846 & $1.0847(10)$ & $\mathrm{E}$ & 98 & 1.0846 & $\mathrm{~T}$ & 99 \\
\hline & & $=\mathrm{C}-\mathrm{H}_{\text {trans }}(C-C)$ & 1.0798 & $1.0793(10)$ & $\mathrm{E}$ & 98 & 1.0799 & $\mathrm{~T}$ & 99 \\
\hline & & $=\mathrm{C}-\mathrm{H}_{c i s}(C-C)$ & 1.0822 & $1.0819(10)$ & $\mathrm{E}$ & 98 & 1.0823 & $\mathrm{~T}$ & 99 \\
\hline & & $\mathrm{C}-\mathrm{C}$ & 1.4550 & $1.4539(10)$ & $\mathrm{E}$ & 98 & 1.4548 & $\mathrm{~T}$ & 99 \\
\hline & & $\mathrm{C}=\mathrm{C}$ & 1.3371 & $1.3376(10)$ & $\mathrm{E}$ & 98 & 1.3377 & $\mathrm{~T}$ & 99 \\
\hline \multirow[t]{2}{*}{$\mathrm{C}_{6} \mathrm{H}_{6}$} & benzene & $\mathrm{C}-\mathrm{H}$ & 1.0812 & $1.0802(20)$ & $\mathrm{E}$ & 107 & & & \\
\hline & & $\mathrm{C}-\mathrm{C}$ & 1.3909 & $1.3914(10)$ & $\mathrm{E}$ & 107 & & & \\
\hline \multirow[t]{3}{*}{$\mathrm{C}_{8} \mathrm{H}_{8}$} & 1,3,5,7-cyclooctatetraene & $\mathrm{C}-\mathrm{H}$ & 1.0850 & $1.0794(10)$ & $\mathrm{E}$ & 108 & 1.0879 & $\mathrm{~T}$ & 109 \\
\hline & & $\mathrm{C}-\mathrm{C}$ & 1.4694 & $1.4702(5)$ & $\mathrm{E}$ & 108 & 1.4668 & $\mathrm{~T}$ & 109 \\
\hline & & $\mathrm{C}=\mathrm{C}$ & 1.3380 & $1.3371(10)$ & $\mathrm{E}$ & 108 & 1.3407 & $\mathrm{~T}$ & 109 \\
\hline
\end{tabular}

${ }^{a}$ Sources are either $(\mathrm{T})$ theoretical data at the $\operatorname{CCSD}(\mathrm{T})$ level with large basis sets, typically extrapolated to the complete basis set limit, and corrected for core-valence correlation or (E) combined experimental and theoretical determinations. See main text for references cited here. 
Table S4: Atomization energy corrections for MP2 geometry errors $(\mathrm{kcal} / \mathrm{mol})^{a}$

\begin{tabular}{|c|c|c|c|c|c|c|c|c|c|}
\hline \multirow{2}{*}{\multicolumn{2}{|c|}{$\begin{array}{l}\text { Level of reference geometry }(\mathrm{G}) \\
\text { Energy model }(m)\end{array}$}} & \multirow[b]{2}{*}{ Molecule $^{b}$} & \multicolumn{3}{|c|}{ B3LYP/cc-pVTZ } & \multicolumn{2}{|c|}{$\mathrm{B}_{6}$} & \multirow{2}{*}{$\frac{\mathrm{A}}{\mathrm{A}}$} & \multirow[b]{2}{*}{$\operatorname{ATOMIC}(\mathrm{hc})$} \\
\hline & & & $\mathrm{A}$ & $\mathrm{B}_{5}$ & $\mathrm{~B}_{6}$ & $\mathrm{~B}_{6}$ & $\mathrm{~A}$ & & \\
\hline $\mathrm{CH}_{4}$ & methane & 1 & -0.01 & 0.01 & 0.00 & 0.01 & 0.00 & 0.00 & $0.01 \pm 0.01$ \\
\hline $\mathrm{C}_{2} \mathrm{H}_{2}$ & acetylene & 2 & 0.05 & 0.05 & 0.07 & 0.11 & 0.09 & 0.09 & $0.06 \pm 0.04$ \\
\hline $\mathrm{C}_{2} \mathrm{H}_{4}$ & ethylene & 3 & -0.03 & -0.02 & -0.01 & 0.01 & 0.00 & 0.00 & $0.01 \pm 0.02$ \\
\hline $\mathrm{C}_{2} \mathrm{H}_{6}$ & ethane & 4 & -0.02 & -0.01 & -0.01 & 0.01 & 0.00 & 0.00 & $0.01 \pm 0.02$ \\
\hline $\mathrm{C}_{3} \mathrm{H}_{4}$ & allene & 5 & -0.08 & -0.07 & -0.07 & 0.01 & 0.00 & 0.00 & $0.01 \pm 0.03$ \\
\hline $\mathrm{C}_{3} \mathrm{H}_{4}$ & cyclopropene & 6 & -0.02 & 0.00 & 0.02 & 0.05 & 0.02 & 0.03 & $0.02 \pm 0.04$ \\
\hline $\mathrm{C}_{3} \mathrm{H}_{4}$ & propyne & 7 & 0.05 & 0.07 & 0.07 & 0.13 & 0.10 & 0.11 & $0.08 \pm 0.06$ \\
\hline $\mathrm{C}_{3} \mathrm{H}_{6}$ & cyclopropane & 8 & -0.03 & -0.01 & -0.01 & 0.02 & 0.00 & 0.00 & $0.02 \pm 0.04$ \\
\hline $\mathrm{C}_{3} \mathrm{H}_{8}$ & propane & 9 & -0.06 & -0.05 & -0.05 & 0.01 & -0.01 & 0.00 & $0.02 \pm 0.04$ \\
\hline $\mathrm{C}_{4} \mathrm{H}_{2}$ & 1,3-butadiyne & 10 & 0.20 & 0.19 & 0.23 & 0.33 & 0.28 & 0.29 & $0.21 \pm 0.13$ \\
\hline $\mathrm{C}_{4} \mathrm{H}_{4}$ & bicyclo[1.1.0]but-1(3)-ene & 11 & 0.03 & 0.03 & 0.05 & 0.27 & 0.25 & 0.26 & $0.06 \pm 0.07$ \\
\hline $\mathrm{C}_{4} \mathrm{H}_{4}$ & butatriene & 12 & -0.10 & -0.10 & -0.11 & 0.01 & 0.00 & 0.01 & $0.02 \pm 0.04$ \\
\hline $\mathrm{C}_{4} \mathrm{H}_{4}$ & cyclobutadiene & 13 & -0.20 & -0.21 & -0.21 & 0.03 & 0.01 & 0.02 & $0.02 \pm 0.05$ \\
\hline $\mathrm{C}_{4} \mathrm{H}_{4}$ & methylenecyclopropene & 14 & 0.01 & 0.03 & 0.04 & 0.07 & 0.04 & 0.04 & $0.06 \pm 0.07$ \\
\hline $\mathrm{C}_{4} \mathrm{H}_{4}$ & tetrahedrane & 15 & 0.02 & 0.04 & 0.04 & 0.04 & 0.01 & 0.02 & $0.07 \pm 0.09$ \\
\hline $\mathrm{C}_{4} \mathrm{H}_{6}$ & 1,3-butadiene & 16 & -0.05 & -0.03 & -0.02 & 0.03 & 0.00 & 0.01 & $0.02 \pm 0.04$ \\
\hline $\mathrm{C}_{4} \mathrm{H}_{6}$ & methylenecyclopropane & 17 & -0.05 & -0.02 & -0.02 & 0.02 & 0.00 & 0.01 & $0.03 \pm 0.05$ \\
\hline $\mathrm{C}_{5} \mathrm{H}_{4}$ & spiropentadiene & 18 & 0.01 & 0.04 & 0.06 & 0.12 & 0.08 & 0.09 & $0.07 \pm 0.09$ \\
\hline $\mathrm{C}_{5} \mathrm{H}_{8}$ & spiropentane & 19 & -0.05 & -0.03 & -0.02 & 0.03 & 0.00 & 0.01 & $0.04 \pm 0.08$ \\
\hline $\mathrm{C}_{5} \mathrm{H}_{12}$ & neopentane & 20 & -0.16 & -0.18 & -0.16 & 0.01 & -0.01 & 0.00 & $0.03 \pm 0.06$ \\
\hline $\mathrm{C}_{6} \mathrm{H}_{4}$ & butalene & 21 & 0.02 & 0.05 & 0.02 & 0.11 & 0.12 & 0.13 & $0.09 \pm 0.10$ \\
\hline $\mathrm{C}_{6} \mathrm{H}_{6}$ & 2,4-hexadiyne & 22 & 0.22 & 0.27 & 0.29 & 0.42 & 0.35 & 0.36 & $0.30 \pm 0.20$ \\
\hline $\mathrm{C}_{6} \mathrm{H}_{6}$ & {$[3]$ radialene } & 23 & -0.03 & -0.01 & 0.00 & 0.04 & 0.02 & 0.03 & $0.04 \pm 0.07$ \\
\hline $\mathrm{C}_{6} \mathrm{H}_{6}$ & benzene & 24 & 0.02 & 0.04 & 0.03 & 0.04 & 0.01 & 0.02 & $0.07 \pm 0.09$ \\
\hline $\mathrm{C}_{6} \mathrm{H}_{6}$ & prismane & 25 & 0.00 & 0.01 & 0.01 & 0.03 & 0.02 & 0.02 & $0.06 \pm 0.11$ \\
\hline $\mathrm{C}_{8} \mathrm{H}_{8}$ & 1,3,5,7-cyclooctatetraene & 26 & -0.17 & -0.16 & -0.14 & 0.15 & 0.11 & 0.12 & $0.05 \pm 0.10$ \\
\hline $\mathrm{C}_{8} \mathrm{H}_{8}$ & cubane & 27 & -0.02 & -0.03 & -0.02 & 0.01 & 0.00 & & $0.07 \pm 0.14$ \\
\hline $\mathrm{C}_{10} \mathrm{H}_{8}$ & naphthalene & 28 & 0.09 & 0.10 & 0.10 & 0.15 & 0.12 & & $0.16 \pm 0.18$ \\
\hline $\mathrm{C}_{10} \mathrm{H}_{8}$ & pentafulvalene & 29 & 0.24 & 0.25 & 0.26 & 0.29 & 0.25 & 0.27 & $0.32 \pm 0.25$ \\
\hline
\end{tabular}


${ }^{a}$ Listed are differences in atomization energy $\Delta_{m / / G}^{g e o}$ (see Eq. (11) for definition) between the reference geometry G and the standard RI-MP2/cc-pVTZ geometry, evaluated using the energy model $m$ given in the second row. A positive value indicates that the chosen geometry is a better approximation to the minimum-energy geometry at the level of the model $m$ used for energy evaluation. Column ATOMIC(hc) lists the actual value and uncertainty used to correct ATOMIC, which itself is always evaluated for RI-MP2/cc-pVTZ geometries. The correction is based on both the atomization energy difference $\left(m=\mathrm{B}_{5}\right)$ between B3LYP and RI-MP2 geometries and an empirical increment system. See text and Table 2 for details.

${ }^{b}$ Column "Molecule" reports the molecule number used in Figure 2. 
Table S5: Zero-point energies of molecules with first-row atoms (HCNOF): Comparison to literature data ${ }^{a}$

\begin{tabular}{|c|c|c|c|c|c|c|c|c|c|c|}
\hline & Molecule & Molecule $^{b}$ & Reference $^{c, d}$ & Other literature data $^{d}$ & RI-MP2 ${ }^{e}$ & corr. $^{f}$ & B3LYP $^{e}$ & corr. $^{f}$ & Average $^{g, h}$ & \\
\hline $\mathrm{CF}_{2}$ & singlet difluoromethylene & 6 & $4.33 \mathrm{~A}$ & & 4.37 & -0.9 & 4.27 & 1.4 & $4.32 \pm 0.11$ & \\
\hline $\mathrm{CF}_{4}$ & tetrafluoromethane & 16 & $10.86 \mathrm{~B}$ & & 10.73 & 1.2 & 10.48 & 3.7 & $10.61 \pm 0.28$ & \\
\hline $\mathrm{CHN}$ & hydrogen cyanide & 14 & $9.95 \mathrm{C}$ & $9.90 \mathrm{D}$ & 9.73 & 2.3 & 10.16 & -2.0 & $9.94 \pm 0.40$ & \\
\hline $\mathrm{CHN}$ & hydrogen isocyanide, $\mathrm{HNC}$ & 13 & $9.71 \mathrm{C}$ & $9.64 \mathrm{D}$ & 9.63 & 0.9 & 9.71 & 0.0 & $9.67 \pm 0.14$ & \\
\hline CHNO & cyanic acid, HOCN & 21 & 13.29 D & & 13.06 & 1.8 & 13.37 & -0.6 & $13.21 \pm 0.34$ & \\
\hline CHNO & formonitrile oxide, $\mathrm{HCNO}$ & 17 & $11.92 \mathrm{D}$ & & 12.17 & -2.1 & 12.26 & -2.8 & $12.21 \pm 0.16$ & $\mathrm{X}$ \\
\hline CHNO & isocyanic acid, $\mathrm{HNCO}$ & 19 & $13.18 \mathrm{D}$ & $13.37 \mathrm{~B}$ & 13.10 & 0.6 & 13.24 & -0.5 & $13.17 \pm 0.21$ & \\
\hline CHNO & isofulminic acid, HONC & 18 & $12.55 \mathrm{D}$ & & 12.51 & 0.3 & 12.61 & -0.5 & $12.56 \pm 0.18$ & \\
\hline $\mathrm{CH}_{2}$ & singlet methylene & 15 & $10.33 \mathrm{C}$ & & 10.49 & -1.5 & 10.28 & 0.5 & $10.38 \pm 0.24$ & \\
\hline $\mathrm{CH}_{2} \mathrm{~F}_{2}$ & difluoromethane & 29 & $20.5 \mathrm{E}$ & & 20.63 & -0.6 & 20.27 & 1.1 & $20.45 \pm 0.43$ & \\
\hline $\mathrm{CH}_{2} \mathrm{O}$ & formaldehyde & 24 & $16.52 \mathrm{C}$ & $16.52 \mathrm{D} ; 16.50 \mathrm{~A} ; 16.6 \mathrm{E}$ & 16.57 & -0.3 & 16.46 & 0.4 & $16.51 \pm 0.22$ & \\
\hline $\mathrm{CH}_{2} \mathrm{O}_{2}$ & dioxirane & 28 & $20.19 \mathrm{D}$ & & 20.30 & -0.5 & 20.14 & 0.3 & $20.22 \pm 0.28$ & \\
\hline $\mathrm{CH}_{2} \mathrm{O}_{2}$ & formic acid & 30 & $21.05 \mathrm{D}$ & & 20.97 & 0.4 & 20.93 & 0.6 & $20.95 \pm 0.20$ & \\
\hline $\mathrm{CH}_{3} \mathrm{NO}$ & formamide & 35 & $28.15 \mathrm{D}$ & & 28.01 & 0.5 & 28.07 & 0.3 & $28.04 \pm 0.27$ & \\
\hline $\mathrm{CH}_{4}$ & methane & 33 & $27.71 \mathrm{C}$ & $27.71 \mathrm{D} ; 27.74 \mathrm{~A}$ & 27.92 & -0.8 & 27.67 & 0.1 & $27.80 \pm 0.41$ & \\
\hline $\mathrm{CH}_{4} \mathrm{O}$ & methanol & 37 & $31.83 \mathrm{D}$ & & 32.02 & -0.6 & 31.69 & 0.4 & $31.85 \pm 0.50$ & \\
\hline $\mathrm{CO}$ & carbon monoxide & 2 & $3.09 \mathrm{C}$ & & 2.97 & 3.9 & 3.13 & -1.2 & $3.05 \pm 0.14$ & \\
\hline $\mathrm{CO}_{2}$ & carbon dioxide & 10 & $7.24 \mathrm{C}$ & $7.25 \mathrm{~A}$ & 7.10 & 1.9 & 7.26 & -0.3 & $7.18 \pm 0.17$ & \\
\hline $\mathrm{C}_{2} \mathrm{H}_{2}$ & acetylene & 23 & $16.44 \mathrm{C}$ & $16.39 \mathrm{D} ; 16.44 \mathrm{~A}$ & 16.32 & 0.7 & 16.76 & -1.9 & $16.54 \pm 0.46$ & \\
\hline $\mathrm{C}_{2} \mathrm{H}_{2} \mathrm{O}$ & ketene & 26 & $19.49 \mathrm{~B}$ & & 19.47 & 0.1 & 19.57 & -0.4 & $19.52 \pm 0.23$ & \\
\hline $\mathrm{C}_{2} \mathrm{H}_{3} \mathrm{~N}$ & acetonitrile & 34 & $27.99 \mathrm{D}$ & & 27.89 & 0.4 & 28.04 & -0.2 & $27.96 \pm 0.34$ & \\
\hline $\mathrm{C}_{2} \mathrm{H}_{4}$ & ethylene & 36 & $31.60 \mathrm{C}$ & $31.47 \mathrm{D} ; 31.54 \mathrm{~A} ; 31.5 \mathrm{E}$ & 31.64 & -0.1 & 31.60 & -0.0 & $31.62 \pm 0.28$ & \\
\hline $\mathrm{C}_{2} \mathrm{H}_{4} \mathrm{O}$ & acetaldehyde & 40 & $34.4 \mathrm{E}$ & & 34.46 & -0.2 & 34.28 & 0.3 & $34.37 \pm 0.41$ & \\
\hline $\mathrm{C}_{2} \mathrm{H}_{5} \mathrm{NO}_{2}$ & glycine & 46 & $49.5 \mathrm{E}$ & & 49.53 & -0.1 & 49.38 & 0.2 & $49.45 \pm 0.51$ & \\
\hline $\mathrm{C}_{2} \mathrm{H}_{6}$ & ethane & 43 & $46.39 \mathrm{~F}$ & $46.28 \mathrm{D} ; 46.34 \mathrm{~A}$ & 46.54 & -0.3 & 46.18 & 0.5 & $46.36 \pm 0.64$ & \\
\hline $\mathrm{C}_{2} \mathrm{H}_{6} \mathrm{O}$ & dimethyl ether & 47 & $49.54 \mathrm{D}$ & & 49.74 & -0.4 & 49.23 & 0.6 & $49.49 \pm 0.78$ & \\
\hline $\mathrm{C}_{3} \mathrm{H}_{4}$ & allene & 39 & $34.08 \mathrm{G}$ & & 34.09 & -0.0 & 34.11 & -0.1 & $34.10 \pm 0.28$ & \\
\hline $\mathrm{C}_{3} \mathrm{H}_{4}$ & propyne & 41 & $34.52 \mathrm{G}$ & & 34.29 & 0.7 & 34.45 & 0.2 & $34.37 \pm 0.40$ & \\
\hline $\mathrm{C}_{3} \mathrm{H}_{6}$ & propene & 45 & $49.36 \mathrm{G}$ & & 49.44 & -0.2 & 49.29 & 0.1 & $49.37 \pm 0.51$ & \\
\hline
\end{tabular}


Table S5, contd.

\begin{tabular}{|c|c|c|c|c|c|c|c|c|c|c|}
\hline & Molecule & Molecule $^{b}$ & Reference $^{c, d}$ & Other literature data ${ }^{d}$ & RI-MP2 $2^{e}$ & corr. $^{f}$ & $\mathrm{~B} 3 \mathrm{LYP}^{e}$ & corr. $^{f}$ & Average $^{g, h}$ & \\
\hline $\mathrm{C}_{3} \mathrm{H}_{8}$ & propane & 50 & $64.02 \mathrm{~A}$ & $64.20 \mathrm{G}$ & 64.35 & -0.5 & 63.94 & 0.1 & $64.14 \pm 0.82$ & \\
\hline $\mathrm{C}_{4} \mathrm{H}_{4} \mathrm{~N}_{2}$ & pyrimidine & 44 & $47.8 \mathrm{E}$ & & 47.65 & 0.3 & 47.72 & 0.2 & $47.68 \pm 0.44$ & \\
\hline $\mathrm{C}_{4} \mathrm{H}_{4} \mathrm{O}$ & furan & 42 & $43.35 \mathrm{~A}$ & $43.4 \mathrm{E}$ & 43.23 & 0.3 & 43.37 & -0.0 & $43.30 \pm 0.45$ & \\
\hline $\mathrm{C}_{5} \mathrm{H}_{5} \mathrm{~N}$ & pyridine & 48 & $55.0 \mathrm{E}$ & & 54.87 & 0.2 & 55.01 & -0.0 & $54.94 \pm 0.54$ & \\
\hline $\mathrm{C}_{6} \mathrm{H}_{6}$ & benzene & 49 & $62.14 \mathrm{H}$ & & 61.94 & 0.3 & 62.31 & -0.3 & $62.13 \pm 0.77$ & \\
\hline FH & hydrogen fluoride & 7 & $5.85 \mathrm{C}$ & & 5.86 & -0.1 & 5.78 & 1.1 & $5.82 \pm 0.10$ & \\
\hline $\mathrm{F}_{2}$ & fluorine & 1 & $1.30 \mathrm{C}$ & & 1.42 & -8.8 & 1.49 & -12.7 & $1.46 \pm 0.06$ & $\mathrm{X}$ \\
\hline $\mathrm{F}_{2} \mathrm{O}$ & difluorine monoxide, FOF & 3 & $3.2 \mathrm{E}$ & & 3.35 & -4.3 & 3.39 & -5.5 & $3.37 \pm 0.06$ & $\mathrm{X}$ \\
\hline $\mathrm{HNO}$ & nitroxyl, HNO & 11 & $8.55 \mathrm{C}$ & $8.57 \mathrm{~A}$ & 8.55 & 0.0 & 8.59 & -0.5 & $8.57 \pm 0.10$ & \\
\hline $\mathrm{H}_{2}$ & hydrogen & 8 & $6.20 \mathrm{C}$ & & 6.34 & -2.3 & 6.25 & -0.8 & $6.30 \pm 0.12$ & \\
\hline $\mathrm{H}_{2} \mathrm{~N}_{2}$ & diazene (trans) & 25 & $17.46 \mathrm{D}$ & $17.45 \mathrm{~A} ; 17.53 \mathrm{~B}$ & 17.52 & -0.3 & 17.58 & -0.7 & $17.55 \pm 0.19$ & \\
\hline $\mathrm{H}_{2} \mathrm{~N}_{2} \mathrm{O}$ & nitrosamine & 27 & $20.03 \mathrm{D}$ & & 20.06 & -0.1 & 20.01 & 0.1 & $20.04 \pm 0.19$ & \\
\hline $\mathrm{H}_{2} \mathrm{O}_{2}$ & hydrogen peroxide & 22 & $16.37 \mathrm{D}$ & $16.25 \mathrm{I}$ & 16.42 & -0.3 & 16.42 & -0.3 & $16.42 \pm 0.14$ & \\
\hline $\mathrm{H}_{3} \mathrm{~N}$ & ammonia & 31 & $21.27 \mathrm{C}$ & $21.29 \mathrm{D} ; 21.22 \mathrm{~A}$ & 21.41 & -0.6 & 21.26 & 0.0 & $21.33 \pm 0.28$ & \\
\hline $\mathrm{H}_{3} \mathrm{NO}$ & hydroxylamine & 32 & $24.97 \mathrm{D}$ & & 25.09 & -0.5 & 24.94 & 0.1 & $25.01 \pm 0.31$ & \\
\hline $\mathrm{H}_{4} \mathrm{~N}_{2}$ & hydrazine & 38 & $33.08 \mathrm{D}$ & & 33.30 & -0.6 & 33.03 & 0.1 & $33.16 \pm 0.46$ & \\
\hline $\mathrm{N}_{2}$ & nitrogen & 4 & $3.36 \mathrm{C}$ & & 3.08 & 9.2 & 3.47 & -3.0 & $3.27 \pm 0.32$ & \\
\hline $\mathrm{N}_{2} \mathrm{O}$ & nitrous oxide & 9 & $6.80 \mathrm{~A}$ & $6.78 \mathrm{~B}$ & 6.62 & 2.7 & 6.96 & -2.3 & $6.79 \pm 0.31$ & \\
\hline $\mathrm{O}_{3}$ & ozone & 5 & $4.15 \mathrm{C}$ & $4.22 \mathrm{~A}$ & 5.81 & -28.6 & 4.51 & -8.0 & $5.16 \pm 1.02$ & \\
\hline
\end{tabular}


$b \quad$ Column "Molecule" reports the molecule number used in Figure 3.

c Selected best estimate of true anharmonic zero-point energy.

$d \quad$ Literature references (see main text) are: Ref. 125 (A), Ref. 126 (B), Ref. 65 (C), Ref. 124 (D), Ref. 128 (E), Ref. 123 (F), Ref. 43 (G), Ref. 46 (H), Ref. 127 (I). The compilation in Ref. 65 (C) quotes both experimental and theoretical values in some cases; experimental values have always been given preference. Ref. 128 (E) reports two "best estimates" in Table 3, the average of which has been quoted here. The two "best estimates" are reported to one decimal place (in $\mathrm{kJ} / \mathrm{mol}$ ), and differences range from 0.0 to 0.9 $\mathrm{kJ} / \mathrm{mol}$. Hence averages in $\mathrm{kcal} / \mathrm{mol}$ are quoted here to one decimal place only. See text for more details.

e RI-MP2 (standard in ATOMIC) and B3LYP values refer to scaled $(0.98,0.989)$ harmonic zero-point energies evaluated using a cc-pVTZ basis set.

$f \quad$ Percentage correction necessary to match computed values with reference data.

$g$ The average of scaled RI-MP2 and B3LYP data defines the model adopted in ATOMIC(hc), along with uncertainties being estimated as three quarters of the difference plus $0.8 \%$ of the average (see text).

$h \quad$ Deviations from reference data larger than the quoted uncertainty estimates are indicated by "X". 
Table S6: Thermal enthalpy increments $\left(\Delta H^{0}[M, 298.15 K] \text {, in kcal/mol }\right)^{a}$

\begin{tabular}{|c|c|c|c|c|c|c|c|c|c|c|}
\hline & \multirow[t]{2}{*}{ Molecule } & \multicolumn{3}{|c|}{$\mathrm{RRHO}^{b}$} & \multicolumn{3}{|c|}{ Reference data } & \multicolumn{3}{|c|}{ Corrections to RRHO } \\
\hline & & RI-MP2 & 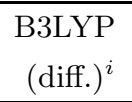 & $\begin{array}{r}\text { exp. } \\
\left(_{\text {(diff. })^{i}}\right.\end{array}$ & 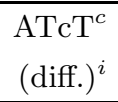 & $\begin{array}{l}\text { Scott }^{d} \\
\text { (diff. }^{i}\end{array}$ & $\begin{array}{r}\text { Dorofeeva }^{e} \\
{\text { (diff. })^{i}}\end{array}$ & Value $^{f}$ & Type $^{g}$ & Note $^{h}$ \\
\hline $\mathrm{CH}_{4}$ & methane & 2.39 & 0.00 & 0.01 & 0.00 & 0.00 & & 0 & & \\
\hline $\mathrm{C}_{2} \mathrm{H}_{2}$ & acetylene & 2.41 & -0.05 & -0.02 & -0.02 & & & 0 & & \\
\hline $\mathrm{C}_{2} \mathrm{H}_{4}$ & ethylene & 2.51 & -0.01 & 0.00 & -0.01 & & & 0 & & \\
\hline $\mathrm{C}_{2} \mathrm{H}_{6}$ & ethane & 2.79 & 0.00 & 0.01 & 0.03 & 0.05 & & 0.05 & $\mathrm{HR}$ & $\mathrm{A}$ \\
\hline $\mathrm{C}_{3} \mathrm{H}_{4}$ & allene & 3.01 & -0.03 & 0.00 & -0.01 & & & 0 & & \\
\hline $\mathrm{C}_{3} \mathrm{H}_{4}$ & cyclopropene & 2.70 & -0.01 & & 0.00 & & 0.02 & 0 & & \\
\hline $\mathrm{C}_{3} \mathrm{H}_{4}$ & propyne & 3.14 & -0.06 & -0.03 & -0.04 & & & 0 & & \\
\hline $\mathrm{C}_{3} \mathrm{H}_{6}$ & cyclopropane & 2.72 & 0.00 & 0.01 & -0.01 & & 0.01 & 0 & & \\
\hline $\mathrm{C}_{3} \mathrm{H}_{6}$ & propene & 3.18 & -0.01 & & 0.04 & & & 0.05 & HR & this work \\
\hline $\mathrm{C}_{3} \mathrm{H}_{8}$ & propane & 3.46 & -0.01 & -0.01 & 0.04 & 0.06 & & 0.09 & HR & A \\
\hline $\mathrm{C}_{4} \mathrm{H}_{2}$ & 1,3-butadiyne & 3.45 & -0.13 & -0.03 & -0.02 & & & 0 & & \\
\hline $\mathrm{C}_{4} \mathrm{H}_{6}$ & 1,3-butadiene & 3.56 & -0.04 & -0.01 & 0.04 & & & 0.01 & $\mathrm{HR}$ & this work \\
\hline $\mathrm{C}_{4} \mathrm{H}_{6}$ & 2-butyne & 4.32 & -0.11 & -0.07 & -0.36 & & & -0.30 & FR & \\
\hline $\mathrm{C}_{4} \mathrm{H}_{6}$ & cyclobutene & 3.04 & -0.04 & & -0.06 & & -0.04 & 0 & & \\
\hline $\mathrm{C}_{4} \mathrm{H}_{8}$ & cyclobutane & 3.17 & 0.05 & 0.06 & 0.05 & & 0.06 & 0 & & \\
\hline $\mathrm{C}_{4} \mathrm{H}_{8}$ & isobutene & 3.98 & -0.04 & -0.09 & 0.06 & & & 0.11 & HR & this work \\
\hline $\mathrm{C}_{4} \mathrm{H}_{10}$ & isobutane & 4.21 & -0.01 & & 0.05 & 0.09 & & 0.11 & HR & $\mathrm{A}$ \\
\hline $\mathrm{C}_{4} \mathrm{H}_{10}$ & $n$-butane & 4.26 & -0.01 & 0.06 & 0.31 & 0.45 & & 0.38 & $\mathrm{CA}+\mathrm{HR}$ & $\mathrm{A}$ \\
\hline $\mathrm{C}_{5} \mathrm{H}_{6}$ & 1,3-cyclopentadiene & 3.26 & -0.05 & & -0.04 & & -0.03 & 0 & & \\
\hline $\mathrm{C}_{5} \mathrm{H}_{8}$ & isoprene & 4.33 & -0.06 & & 0.09 & & & 0.06 & $\mathrm{HR}$ & this work \\
\hline $\mathrm{C}_{5} \mathrm{H}_{10}$ & cyclopentane & 3.91 & -0.01 & & -0.34 & & -0.32 & -0.30 & FPR & \\
\hline $\mathrm{C}_{5} \mathrm{H}_{12}$ & neopentane & 5.01 & -0.03 & & 0.50 & 0.53 & & 0.06 & $\mathrm{HR}$ & $\mathrm{A}$ \\
\hline $\mathrm{C}_{5} \mathrm{H}_{12}$ & $n$-pentane & 5.09 & -0.01 & & 0.66 & 0.69 & & 0.65 & $\mathrm{CA}+\mathrm{HR}$ & $\mathrm{A}$ \\
\hline $\mathrm{C}_{6} \mathrm{H}_{4}$ & ortho-benzyne & 3.33 & 0.07 & & 0.08 & & & 0 & & \\
\hline $\mathrm{C}_{6} \mathrm{H}_{6}$ & benzene & 3.43 & -0.06 & -0.04 & -0.05 & & & 0 & & \\
\hline $\mathrm{C}_{6} \mathrm{H}_{8}$ & 1,3-cyclohexadiene & 3.90 & -0.03 & & & & -0.01 & 0 & & \\
\hline $\mathrm{C}_{6} \mathrm{H}_{8}$ & 1,4-cyclohexadiene & 3.98 & -0.07 & & & & -0.02 & 0 & & \\
\hline
\end{tabular}


Table S6, contd.

\begin{tabular}{|c|c|c|c|c|c|c|c|c|c|c|}
\hline & \multirow[t]{2}{*}{ Molecule } & \multicolumn{3}{|c|}{$\mathrm{RRHO}^{b}$} & \multicolumn{3}{|c|}{ Reference data } & \multicolumn{3}{|c|}{ Corrections to RRHO } \\
\hline & & RI-MP2 & $\begin{array}{r}\text { B3LYP } \\
\text { (diff.) }^{i}\end{array}$ & $\begin{array}{r}\text { exp. } \\
(\text { diff. })^{i}\end{array}$ & $\begin{array}{l}\text { ATcT }^{c} \\
\text { (diff.) }^{i}\end{array}$ & $\begin{array}{l}\mathrm{Scott}^{d} \\
(\text { diff. })^{i}\end{array}$ & $\begin{array}{r}\text { Dorofeeva }^{e} \\
{\text { (diff. })^{i}}\end{array}$ & Value $^{f}$ & Type $^{g}$ & Note $^{h}$ \\
\hline $\mathrm{C}_{6} \mathrm{H}_{10}$ & cyclohexene & 4.10 & -0.02 & & & & 0.03 & 0 & & \\
\hline $\mathrm{C}_{6} \mathrm{H}_{12}$ & cyclohexane & 4.23 & -0.01 & -0.05 & -0.07 & & -0.04 & 0 & & \\
\hline $\mathrm{C}_{6} \mathrm{H}_{14}$ & 3-methylpentane & 5.92 & -0.02 & & & 0.31 & & 0.50 & $\mathrm{CA}+\mathrm{HR}$ & $\mathrm{A}$ \\
\hline $\mathrm{C}_{6} \mathrm{H}_{14}$ & $n$-hexane & 5.95 & -0.03 & & 0.88 & 0.91 & & 0.91 & $\mathrm{CA}+\mathrm{HR}$ & $\mathrm{A}$ \\
\hline $\mathrm{C}_{7} \mathrm{H}_{8}$ & 2,5-norbornadiene & 3.72 & -0.04 & & 0.04 & & & 0 & & \\
\hline $\mathrm{C}_{7} \mathrm{H}_{8}$ & toluene & 4.58 & -0.07 & & -0.32 & & & -0.23 & $\mathrm{FR}$ & \\
\hline $\mathrm{C}_{7} \mathrm{H}_{16}$ & $n$-heptane & 6.81 & -0.04 & & 1.09 & 1.13 & & 1.15 & $\mathrm{CA}+\mathrm{HR}$ & $\mathrm{A}$ \\
\hline $\mathrm{C}_{8} \mathrm{H}_{8}$ & 1,3,5,7-cyclooctatetraene & 4.96 & -0.13 & & & & -0.04 & 0 & & \\
\hline $\mathrm{C}_{8} \mathrm{H}_{8}$ & styrene & 4.95 & -0.08 & & 0.03 & & & 0.06 & $\mathrm{HR}$ & this work \\
\hline $\mathrm{C}_{8} \mathrm{H}_{18}$ & $n$-octane & 7.68 & -0.05 & & 1.31 & 1.35 & & 1.40 & $\mathrm{CA}+\mathrm{HR}$ & $\mathrm{A}$ \\
\hline
\end{tabular}


a $\Delta H^{0}[M, 298.15 \mathrm{~K}]$ is the difference in enthalpy of molecule $M$ between $T=298.15 \mathrm{~K}$ and $T=0 \mathrm{~K}$. See Eq. (4).

$b \quad$ Rigid-rotor harmonic-oscillator treatment with either scaled $\left(f_{\text {scal }}=0.98\right)$ harmonic RI-MP2/cc-pVTZ, scaled $\left(f_{\text {scal }}=0.989\right)$ harmonic B3LYP/cc-pVTZ, or experimental fundamental frequencies, taken from Ref. 131.

c $\quad \Delta H^{0}[M, 298.15 \mathrm{~K}]$ values have been obtained from $\Delta H_{f}^{0}[M, 298.15 \mathrm{~K}]=\Delta H_{f}^{0}[M, 0 \mathrm{~K}]+\Delta H^{0}[M, 298.15 \mathrm{~K}]-\sum_{k \in M}^{a t o m s} \Delta H^{0}(k, 298.15 \mathrm{~K})$ (compare Eq. (3)), using $\Delta H^{0}(k, 298.15 \mathrm{~K})$ values taken from Ref. 72 and $\Delta H_{f}^{0}[M, 298.15 \mathrm{~K}]$ and $\Delta H_{f}^{0}[M, 0 \mathrm{~K}]$ values as listed in the Active Thermochemical Tables (ATcT version 1.122d, Refs. 48-50).

$d \quad$ Taken from Ref. 134. ${ }^{e}$ Taken from Ref. 135. ${ }^{f}$ The entry "0" signals that no correction is made or necessary.

$g \quad$ FR, FPR: free internal rotor (pseudorotor) correction $(R T / 2=0.3 \mathrm{kcal} / \mathrm{mol}$ replacing actually calculated harmonic oscillator term for that mode, which is $R T$ in the limit of zero frequency); HR: hindered rotor correction; CA: conformational averaging correction. "CA+HR" reports the sum of conformational averaging and hindered-rotor corrections, HR contributions alone amount to (Ref. 43): $n$-butane: $0.13 \mathrm{kcal} / \mathrm{mol}, n$-pentane: $0.19 \mathrm{kcal} / \mathrm{mol}, 3$-methylpentane: $0.24 \mathrm{kcal} / \mathrm{mol}, n$-hexane: $0.24 \mathrm{kcal} / \mathrm{mol}, n$-heptane: 0.28 $\mathrm{kcal} / \mathrm{mol}, n$-octane: $0.35 \mathrm{kcal} / \mathrm{mol}$.

$h$ Values have been taken from Ref. 43 ("A") if available or computed here ("this work"), using the Ayala-Schlegel approach (Ref. 132) at the B3LYP/cc-pVTZ level as implemented in Gaussian. Hindered rotor corrections generally agree to within $0.01 \mathrm{kcal} / \mathrm{mol}$ with those reported in Ref. 43 (Ayala-Schlegel, B3LYP/pc-2), except for neopentane $(0.12$ vs $0.06 \mathrm{kcal} / \mathrm{mol})$ and for $n$-octane (0.33 vs $0.35 \mathrm{kcal} / \mathrm{mol})$.

i Data are expressed as difference with respect to the RRHO treatment with scaled harmonic RI-MP2/cc-pVTZ frequencies (first column). 
Table S7: Auxiliary data for atomization energies and enthalpies of formation ${ }^{a}$

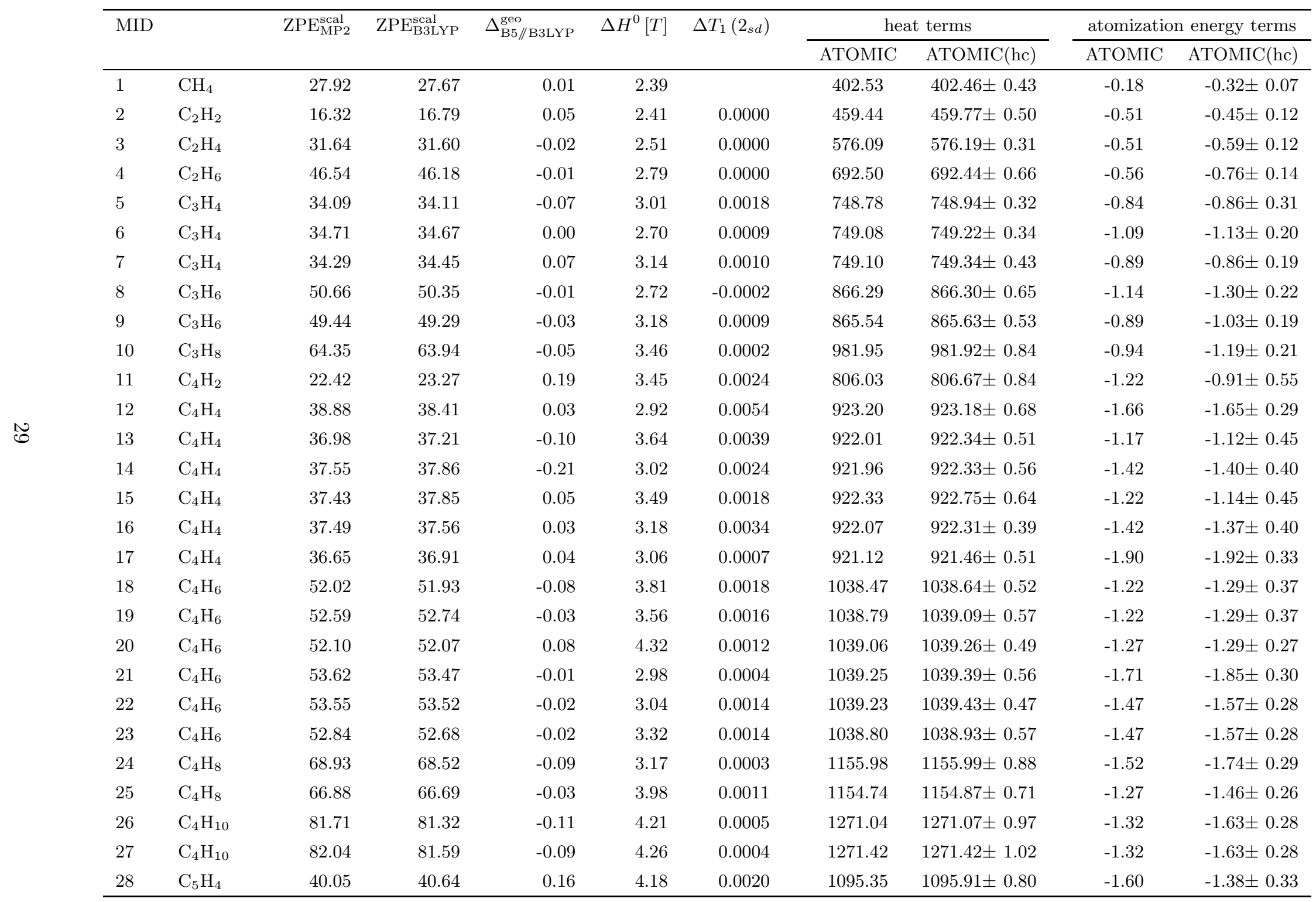


Table S7, contd.

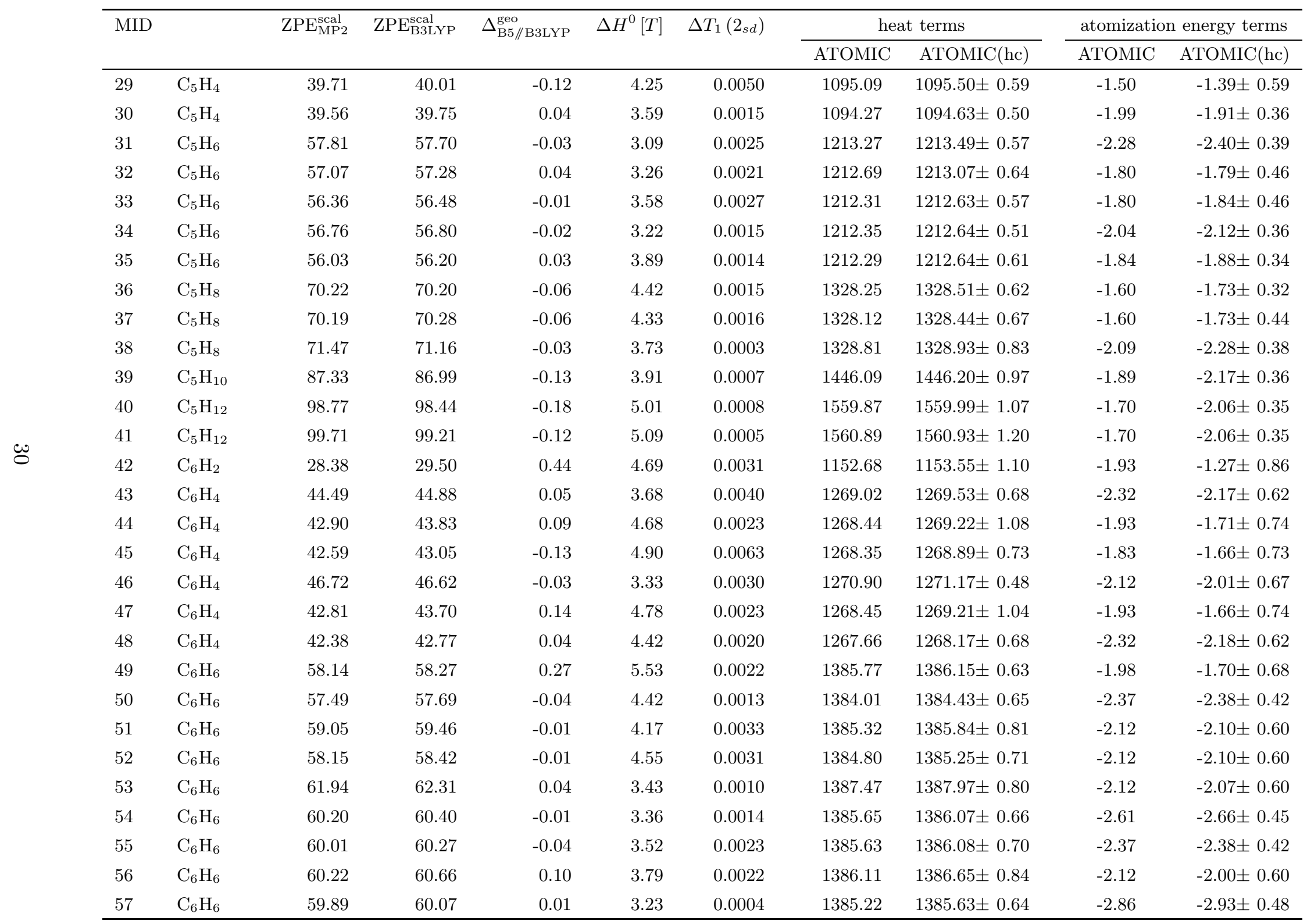


Table S7, contd.

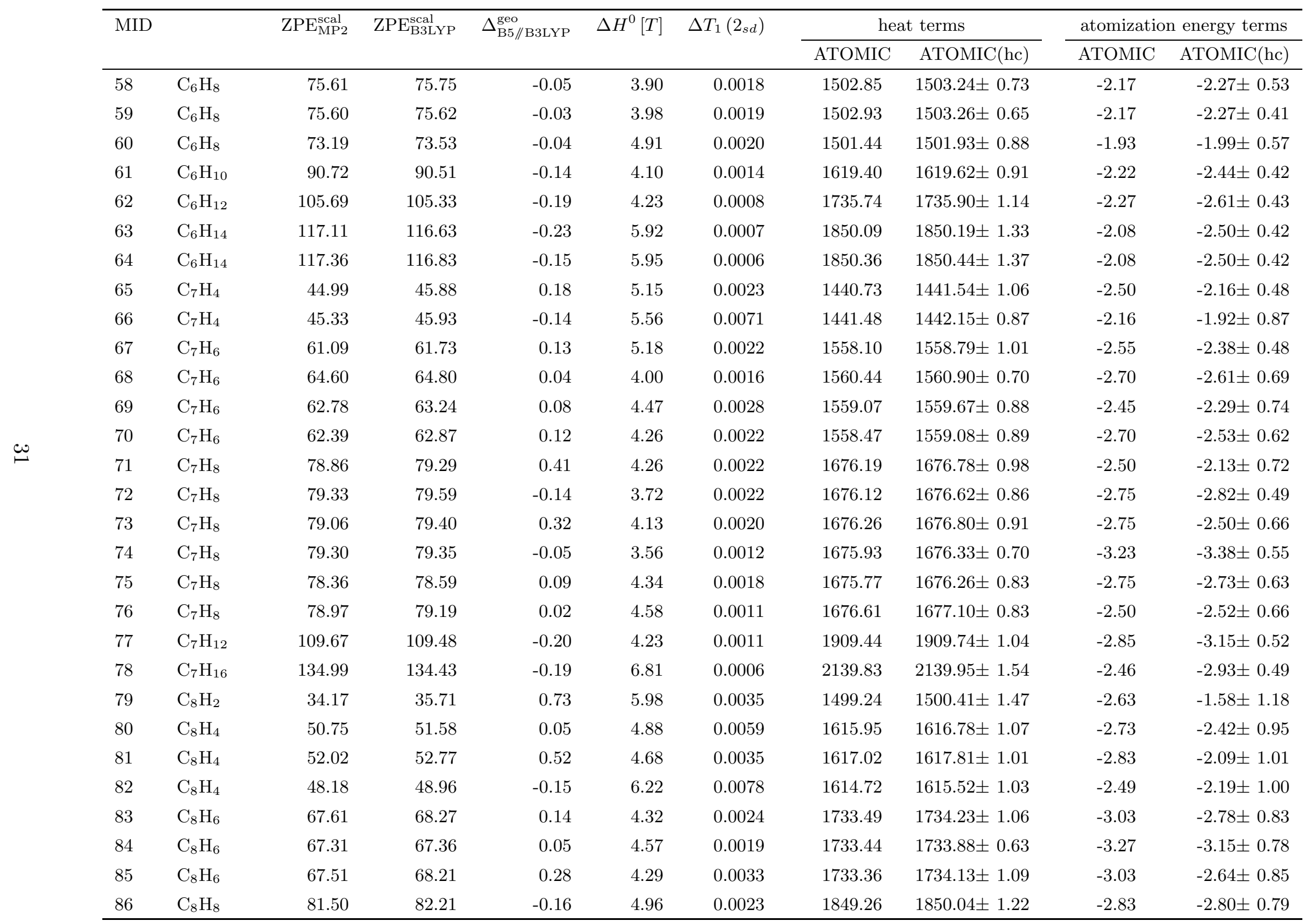


Table S7, contd.

\begin{tabular}{|c|c|c|c|c|c|c|c|c|c|c|}
\hline \multirow[t]{2}{*}{ MID } & & \multirow[t]{2}{*}{$\mathrm{ZPE}_{\mathrm{MP} 2}^{\mathrm{scal}}$} & \multirow[t]{2}{*}{$\mathrm{ZPE}_{\mathrm{B} 3 \mathrm{LYP}}^{\mathrm{scal}}$} & \multirow[t]{2}{*}{$\Delta_{\mathrm{B} 5 / / \mathrm{B} 3 \mathrm{LYP}}^{\mathrm{geo}}$} & \multirow[t]{2}{*}{$\Delta H^{0}[T]$} & \multirow[t]{2}{*}{$\Delta T_{1}\left(2_{s d}\right)$} & \multicolumn{2}{|c|}{ heat terms } & \multicolumn{2}{|c|}{ atomization energy terms } \\
\hline & & & & & & & ATOMIC & ATOMIC(hc) & ATOMIC & ATOMIC(hc) \\
\hline 87 & $\mathrm{C}_{8} \mathrm{H}_{8}$ & 82.96 & 83.22 & 0.02 & 4.48 & 0.0013 & 1850.24 & $1850.79 \pm 0.90$ & -3.08 & $-3.07 \pm 0.75$ \\
\hline 88 & $\mathrm{C}_{8} \mathrm{H}_{8}$ & 82.05 & 82.54 & -0.03 & 3.56 & 0.0007 & 1848.42 & $1849.08 \pm 1.04$ & -3.81 & $-3.92 \pm 0.64$ \\
\hline 89 & $\mathrm{C}_{8} \mathrm{H}_{8}$ & 81.23 & 82.05 & 0.02 & 4.95 & 0.0032 & 1848.99 & $1849.82 \pm 1.29$ & -2.83 & $-2.79 \pm 0.79$ \\
\hline 90 & $\mathrm{C}_{8} \mathrm{H}_{8}$ & 82.15 & 82.63 & -0.01 & 4.95 & 0.0014 & 1849.90 & $1850.56 \pm 1.05$ & -2.83 & $-2.80 \pm 0.79$ \\
\hline 91 & $\mathrm{C}_{8} \mathrm{H}_{10}$ & 93.73 & 94.28 & -0.05 & 6.30 & 0.0023 & 1964.07 & $1964.77 \pm 1.21$ & -2.64 & $-2.69 \pm 0.77$ \\
\hline 92 & $\mathrm{C}_{8} \mathrm{H}_{14}$ & 127.99 & 127.71 & -0.43 & 4.87 & 0.0010 & 2199.39 & $2199.69 \pm 1.26$ & -3.23 & $-3.59 \pm 0.59$ \\
\hline 93 & $\mathrm{C}_{8} \mathrm{H}_{18}$ & 152.63 & 152.02 & -0.22 & 7.68 & 0.0006 & 2429.30 & $2429.45 \pm 1.72$ & -2.84 & $-3.37 \pm 0.57$ \\
\hline 94 & $\mathrm{C}_{9} \mathrm{H}_{20}$ & 170.27 & 169.59 & -0.26 & 8.54 & 0.0007 & 2718.78 & $2718.95 \pm 1.92$ & -3.22 & $-3.80 \pm 0.64$ \\
\hline 95 & $\mathrm{C}_{10} \mathrm{H}_{2}$ & 39.92 & 41.88 & 1.12 & 7.30 & 0.0038 & 1845.76 & $1847.25 \pm 1.83$ & -3.34 & $-1.79 \pm 1.53$ \\
\hline 96 & $\mathrm{C}_{10} \mathrm{H}_{4}$ & 53.25 & 55.03 & 0.50 & 7.41 & 0.0026 & 1960.44 & $1961.85 \pm 1.81$ & -3.34 & $-2.51 \pm 1.34$ \\
\hline 97 & $\mathrm{C}_{10} \mathrm{H}_{8}$ & 90.23 & 90.46 & 0.03 & 5.22 & 0.0032 & 2197.71 & $2198.35 \pm 0.94$ & -3.73 & $-3.58 \pm 1.02$ \\
\hline 98 & $\mathrm{C}_{10} \mathrm{H}_{8}$ & 90.54 & 91.38 & 0.10 & 5.05 & 0.0015 & 2197.84 & $2198.79 \pm 1.39$ & -3.73 & $-3.51 \pm 1.02$ \\
\hline 99 & $\mathrm{C}_{10} \mathrm{H}_{8}$ & 88.16 & 89.18 & 0.25 & 5.63 & 0.0025 & 2196.05 & $2197.08 \pm 1.50$ & -3.73 & $-3.36 \pm 1.04$ \\
\hline 100 & $\mathrm{C}_{10} \mathrm{H}_{10}$ & 101.46 & 101.69 & 0.20 & 7.19 & 0.0023 & 2312.15 & $2312.79 \pm 1.06$ & -3.88 & $-3.73 \pm 0.97$ \\
\hline 101 & $\mathrm{C}_{10} \mathrm{H}_{10}$ & 102.89 & 103.26 & 0.12 & 6.72 & 0.0015 & 2313.11 & $2313.82 \pm 1.16$ & -3.59 & $-3.46 \pm 1.00$ \\
\hline 102 & $\mathrm{C}_{10} \mathrm{H}_{10}$ & 102.72 & 103.10 & -0.09 & 6.12 & 0.0016 & 2312.35 & $2313.06 \pm 1.15$ & -3.78 & $-3.78 \pm 0.87$ \\
\hline 103 & $\mathrm{C}_{10} \mathrm{H}_{10}$ & 102.11 & 102.69 & 0.05 & 6.06 & 0.0029 & 2311.67 & $2312.49 \pm 1.30$ & -3.78 & $-3.73 \pm 0.95$ \\
\hline 104 & $\mathrm{C}_{10} \mathrm{H}_{10}$ & 104.82 & 105.28 & -0.08 & 4.34 & 0.0020 & 2312.66 & $2313.42 \pm 1.21$ & -4.52 & $-4.62 \pm 0.77$ \\
\hline 105 & $\mathrm{C}_{10} \mathrm{H}_{10}$ & 103.95 & 104.13 & 0.00 & 5.58 & 0.0015 & 2313.03 & $2313.65 \pm 1.01$ & -4.03 & $-4.06 \pm 0.90$ \\
\hline 106 & $\mathrm{C}_{10} \mathrm{H}_{10}$ & 104.71 & 105.25 & 0.08 & 5.21 & 0.0025 & 2313.41 & $2314.21 \pm 1.28$ & -4.03 & $-3.98 \pm 0.72$ \\
\hline 107 & $\mathrm{C}_{10} \mathrm{H}_{10}$ & 105.71 & 105.93 & -0.08 & 4.14 & 0.0014 & 2313.35 & $2313.99 \pm 1.04$ & -4.76 & $-4.90 \pm 0.80$ \\
\hline 108 & $\mathrm{C}_{10} \mathrm{H}_{10}$ & 99.17 & 99.74 & 0.37 & 8.08 & 0.0024 & 2310.75 & $2311.56 \pm 1.30$ & -3.39 & $-3.00 \pm 1.08$ \\
\hline 109 & $\mathrm{C}_{10} \mathrm{H}_{10}$ & 103.76 & 104.40 & -0.18 & 5.05 & 0.0025 & 2312.31 & $2313.16 \pm 1.34$ & -4.03 & $-4.06 \pm 0.71$ \\
\hline 110 & $\mathrm{C}_{10} \mathrm{H}_{10}$ & 105.63 & 105.93 & -0.12 & 4.30 & 0.0020 & 2313.44 & $2314.12 \pm 1.10$ & -4.52 & $-4.62 \pm 0.77$ \\
\hline 111 & $\mathrm{C}_{10} \mathrm{H}_{10}$ & 104.76 & 105.17 & -0.12 & 4.99 & 0.0026 & 2313.25 & $2313.98 \pm 1.17$ & -4.03 & $-4.06 \pm 0.71$ \\
\hline 112 & $\mathrm{C}_{10} \mathrm{H}_{12}$ & 114.25 & 115.00 & -0.05 & 7.71 & 0.0024 & 2426.69 & $2427.60 \pm 1.53$ & -3.35 & $-3.40 \pm 0.97$ \\
\hline 113 & $\mathrm{C}_{10} \mathrm{H}_{16}$ & 150.73 & 150.57 & -0.33 & 5.12 & 0.0013 & 2663.06 & $2663.53 \pm 1.35$ & -4.18 & $-4.57 \pm 0.75$ \\
\hline 114 & $\mathrm{C}_{10} \mathrm{H}_{16}$ & 149.81 & 149.59 & -0.50 & 5.48 & 0.0014 & 2662.51 & $2662.95 \pm 1.40$ & -4.18 & $-4.57 \pm 0.75$ \\
\hline 115 & $\mathrm{C}_{10} \mathrm{H}_{18}$ & 163.80 & 163.47 & -0.50 & 6.20 & 0.0012 & 2778.45 & $2778.85 \pm 1.58$ & -3.98 & $-4.46 \pm 0.74$ \\
\hline
\end{tabular}


Table S7, contd.

\begin{tabular}{|c|c|c|c|c|c|c|c|c|c|c|}
\hline MID & & $\mathrm{ZPE}_{\mathrm{MP} 2}^{\mathrm{scal}}$ & $\mathrm{ZPE}_{\mathrm{B} 3 \mathrm{LYP}}^{\text {scal }}$ & $\Delta_{\mathrm{B} 5 / / \mathrm{B} 3 \mathrm{LYP}}^{\mathrm{geo}}$ & $\Delta H^{0}[T]$ & $\Delta T_{1}\left(2_{s d}\right)$ & & t terms & atomizatio & energy terms \\
\hline & & & & & & & ATOMIC & ATOMIC(hc) & ATOMIC & ATOMIC(hc) \\
\hline 116 & $\mathrm{C}_{10} \mathrm{H}_{18}$ & 163.48 & 163.21 & -0.41 & 6.28 & 0.0012 & 2778.22 & $2778.64 \pm 1.55$ & -3.98 & $-4.46 \pm 0.74$ \\
\hline 117 & $\mathrm{C}_{10} \mathrm{H}_{22}$ & 187.89 & 187.13 & -0.29 & 9.42 & 0.0007 & 3008.25 & $3008.44 \pm 2.13$ & -3.59 & $-4.24 \pm 0.71$ \\
\hline 118 & $\mathrm{C}_{11} \mathrm{H}_{24}$ & 205.52 & 204.71 & -0.32 & 10.29 & 0.0007 & 3297.72 & $3297.94 \pm 2.31$ & -3.97 & $-4.67 \pm 0.78$ \\
\hline 119 & $\mathrm{C}_{12} \mathrm{H}_{6}$ & 81.29 & 82.26 & 0.75 & 5.25 & 0.0042 & 2427.02 & $2428.13 \pm 1.41$ & -4.83 & $-3.78 \pm 1.37$ \\
\hline 120 & $\mathrm{C}_{12} \mathrm{H}_{6}$ & 74.34 & 75.12 & 0.14 & 8.07 & 0.0032 & 2422.89 & $2423.90 \pm 1.26$ & -4.83 & $-4.40 \pm 1.28$ \\
\hline 121 & $\mathrm{C}_{12} \mathrm{H}_{8}$ & 97.63 & 98.72 & 0.29 & 5.63 & 0.0020 & 2544.98 & $2546.15 \pm 1.63$ & -4.64 & $-4.14 \pm 1.27$ \\
\hline 122 & $\mathrm{C}_{12} \mathrm{H}_{8}$ & 97.12 & 98.06 & 0.17 & 5.90 & 0.0019 & 2544.75 & $2545.84 \pm 1.52$ & -4.64 & $-4.25 \pm 1.25$ \\
\hline 123 & $\mathrm{C}_{12} \mathrm{H}_{10}$ & 111.76 & 112.53 & 0.05 & 6.35 & 0.0013 & 2661.07 & $2662.09 \pm 1.51$ & -4.44 & $-4.27 \pm 1.22$ \\
\hline 124 & $\mathrm{C}_{12} \mathrm{H}_{12}$ & 125.75 & 126.34 & -0.25 & 6.69 & 0.0018 & 2776.64 & $2777.57 \pm 1.50$ & -4.49 & $-4.49 \pm 1.14$ \\
\hline 125 & $\mathrm{C}_{12} \mathrm{H}_{14}$ & 134.74 & 135.70 & -0.06 & 9.12 & 0.0025 & 2889.30 & $2890.42 \pm 1.86$ & -4.05 & $-4.10 \pm 1.17$ \\
\hline 126 & $\mathrm{C}_{12} \mathrm{H}_{18}$ & 165.08 & 164.90 & -0.10 & 9.76 & 0.0014 & 3122.76 & $3123.32 \pm 1.54$ & -4.40 & $-4.71 \pm 1.00$ \\
\hline 127 & $\mathrm{C}_{12} \mathrm{H}_{26}$ & 223.15 & 222.29 & -0.36 & 11.17 & 0.0007 & 3587.19 & $3587.44 \pm 2.49$ & -4.35 & $-5.11 \pm 0.86$ \\
\hline 128 & $\mathrm{C}_{13} \mathrm{H}_{10}$ & 115.63 & 116.44 & 0.05 & 6.42 & 0.0015 & 2834.74 & $2835.83 \pm 1.58$ & -5.02 & $-4.81 \pm 1.31$ \\
\hline 129 & $\mathrm{C}_{13} \mathrm{H}_{28}$ & 240.77 & 239.93 & -0.39 & 12.04 & 0.0007 & 3876.66 & $3876.97 \pm 2.63$ & -4.73 & $-5.54 \pm 0.93$ \\
\hline 130 & $\mathrm{C}_{14} \mathrm{H}_{8}$ & 104.53 & 105.04 & 0.19 & 6.63 & 0.0022 & 2892.34 & $2893.32 \pm 1.27$ & -5.34 & $-4.84 \pm 1.53$ \\
\hline 131 & $\mathrm{C}_{14} \mathrm{H}_{8}$ & 104.43 & 105.83 & 0.39 & 6.32 & 0.0025 & 2891.93 & $2893.36 \pm 1.92$ & -5.54 & $-4.84 \pm 1.50$ \\
\hline 132 & $\mathrm{C}_{14} \mathrm{H}_{10}$ & 118.87 & 120.25 & 0.20 & 6.80 & 0.0018 & 3008.09 & $3009.51 \pm 2.02$ & -5.35 & $-4.93 \pm 1.45$ \\
\hline 133 & $\mathrm{C}_{14} \mathrm{H}_{10}$ & 119.13 & 120.49 & 0.13 & 6.81 & 0.0015 & 3008.36 & $3009.77 \pm 2.01$ & -5.35 & $-5.00 \pm 1.45$ \\
\hline 134 & $\mathrm{C}_{14} \mathrm{H}_{20}$ & 195.38 & 195.45 & -0.53 & 6.29 & 0.0017 & 3590.29 & $3591.08 \pm 1.65$ & -6.08 & $-6.53 \pm 1.07$ \\
\hline 135 & $\mathrm{C}_{14} \mathrm{H}_{30}$ & 258.39 & 257.50 & -0.42 & 12.92 & 0.0008 & 4166.13 & $4166.47 \pm 2.82$ & -5.11 & $-5.98 \pm 1.00$ \\
\hline 136 & $\mathrm{C}_{15} \mathrm{H}_{32}$ & 276.02 & 275.09 & -0.46 & 13.80 & 0.0008 & 4455.60 & $4455.98 \pm 2.99$ & -5.49 & $-6.41 \pm 1.07$ \\
\hline 137 & $\mathrm{C}_{16} \mathrm{H}_{8}$ & 111.74 & 113.00 & 0.65 & 6.62 & 0.0033 & 3238.99 & $3240.45 \pm 1.88$ & -6.44 & $-5.40 \pm 1.76$ \\
\hline 138 & $\mathrm{C}_{16} \mathrm{H}_{10}$ & 126.53 & 128.02 & 0.25 & 7.39 & 0.0018 & 3355.81 & $3357.38 \pm 2.17$ & -6.25 & $-5.69 \pm 1.68$ \\
\hline 139 & $\mathrm{C}_{16} \mathrm{H}_{10}$ & 126.92 & 128.53 & 0.23 & 7.20 & 0.0018 & 3356.00 & $3357.63 \pm 2.27$ & -6.25 & $-5.71 \pm 1.68$ \\
\hline 140 & $\mathrm{C}_{16} \mathrm{H}_{12}$ & 138.76 & 140.03 & -0.08 & 7.98 & 0.0020 & 3469.86 & $3471.33 \pm 2.11$ & -6.05 & $-5.83 \pm 1.64$ \\
\hline 141 & $\mathrm{C}_{16} \mathrm{H}_{16}$ & 169.67 & 170.44 & -0.42 & 8.18 & 0.0015 & 3703.45 & $3704.68 \pm 1.99$ & -6.15 & $-6.17 \pm 1.50$ \\
\hline 142 & $\mathrm{C}_{16} \mathrm{H}_{16}$ & 169.40 & 170.08 & -0.60 & 8.18 & 0.0015 & 3703.18 & $3704.37 \pm 1.92$ & -6.15 & $-6.17 \pm 1.50$ \\
\hline 143 & $\mathrm{C}_{16} \mathrm{H}_{16}$ & 168.60 & 169.48 & -0.59 & 8.51 & 0.0014 & 3702.71 & $3704.00 \pm 2.06$ & -6.15 & $-6.17 \pm 1.50$ \\
\hline 144 & $\mathrm{C}_{16} \mathrm{H}_{34}$ & 293.64 & 292.63 & -0.49 & 14.67 & 0.0008 & 4745.07 & $4745.47 \pm 3.20$ & -5.87 & $-6.85 \pm 1.14$ \\
\hline
\end{tabular}


Table S7, contd.

\begin{tabular}{|c|c|c|c|c|c|c|c|c|c|c|c|}
\hline & \multirow[t]{2}{*}{ MID } & & \multirow[t]{2}{*}{$\mathrm{ZPE}_{\mathrm{MP} 2}^{\mathrm{scal}}$} & \multirow[t]{2}{*}{$\mathrm{ZPE}_{\mathrm{B} 3 \mathrm{LYP}}^{\mathrm{scal}}$} & \multirow{2}{*}{$\Delta_{\mathrm{B} 5 / / \mathrm{B} 3 \mathrm{LYP}}^{\mathrm{geo}}$} & \multirow[t]{2}{*}{$\Delta H^{0}[T]$} & \multirow[t]{2}{*}{$\Delta T_{1}\left(2_{s d}\right)$} & \multicolumn{2}{|c|}{ heat terms } & \multicolumn{2}{|c|}{ atomization energy terms } \\
\hline & & & & & & & & ATOMIC & ATOMIC(hc) & ATOMIC & ATOMIC(hc) \\
\hline & 145 & $\mathrm{C}_{18} \mathrm{H}_{12}$ & 148.19 & 149.69 & 0.18 & 8.43 & 0.0017 & 3819.20 & $3820.88 \pm 2.36$ & -6.96 & $-6.46 \pm 1.87$ \\
\hline & 146 & $\mathrm{C}_{18} \mathrm{H}_{12}$ & 147.50 & 149.53 & 0.21 & 8.67 & 0.0016 & 3818.75 & $3820.70 \pm 2.75$ & -6.96 & $-6.43 \pm 1.88$ \\
\hline & 147 & $\mathrm{C}_{18} \mathrm{H}_{12}$ & 147.13 & 149.04 & 0.36 & 8.59 & 0.0021 & 3818.30 & $3820.19 \pm 2.65$ & -6.96 & $-6.28 \pm 1.89$ \\
\hline & 148 & $\mathrm{C}_{18} \mathrm{H}_{12}$ & 147.20 & 149.60 & 0.14 & 8.91 & 0.0015 & 3818.69 & $3820.83 \pm 3.03$ & -6.96 & $-6.50 \pm 1.87$ \\
\hline & 149 & $\mathrm{C}_{18} \mathrm{H}_{18}$ & 187.99 & 188.49 & 0.01 & 10.96 & 0.0017 & 4165.24 & $4166.45 \pm 1.97$ & -6.86 & $-6.86 \pm 1.69$ \\
\hline & 150 & $\mathrm{C}_{18} \mathrm{H}_{18}$ & 187.93 & 188.94 & -0.39 & 10.78 & 0.0016 & 4165.01 & $4166.47 \pm 2.34$ & -6.62 & $-6.59 \pm 1.74$ \\
\hline & 151 & $\mathrm{C}_{18} \mathrm{H}_{18}$ & 188.33 & 188.97 & -0.03 & 10.58 & 0.0016 & 4165.20 & $4166.48 \pm 2.07$ & -6.86 & $-6.87 \pm 1.69$ \\
\hline & 152 & $\mathrm{C}_{20}$ & 73.36 & 70.23 & 0.67 & 5.32 & 0.0062 & 3473.28 & $3472.72 \pm 2.94$ & -9.02 & $-7.44 \pm 2.31$ \\
\hline & 153 & $\mathrm{C}_{20} \mathrm{H}_{10}$ & 141.95 & 143.79 & 0.51 & 8.14 & 0.0021 & 4050.89 & $4052.84 \pm 2.56$ & -8.05 & $-7.05 \pm 2.16$ \\
\hline \multirow{8}{*}{$\stackrel{\Perp}{\oplus}$} & 154 & $\mathrm{C}_{20} \mathrm{H}_{12}$ & 155.02 & 157.35 & 0.28 & 9.23 & 0.0018 & 4166.29 & $4168.49 \pm 3.04$ & -7.86 & $-7.17 \pm 2.11$ \\
\hline & 155 & $\mathrm{C}_{20} \mathrm{H}_{14}$ & 169.90 & 171.32 & 0.07 & 9.22 & 0.0019 & 4282.40 & $4284.16 \pm 2.48$ & -7.91 & $-7.55 \pm 2.02$ \\
\hline & 156 & $\mathrm{C}_{20} \mathrm{H}_{20}$ & 220.94 & 221.87 & -0.43 & 6.10 & 0.0021 & 4634.04 & $4635.57 \pm 2.50$ & -9.52 & $-9.80 \pm 1.61$ \\
\hline & 157 & $\mathrm{C}_{20} \mathrm{H}_{20}$ & 217.07 & 217.90 & -0.57 & 7.06 & 0.0024 & 4631.13 & $4632.61 \pm 2.40$ & -9.52 & $-9.80 \pm 1.61$ \\
\hline & 158 & $\mathrm{C}_{20} \mathrm{H}_{36}$ & 315.79 & 315.75 & -1.12 & 17.25 & 0.0016 & 5549.96 & $5551.05 \pm 2.71$ & -7.97 & $-8.92 \pm 1.47$ \\
\hline & 159 & $\mathrm{C}_{24} \mathrm{H}_{12}$ & 171.13 & 173.62 & 0.44 & 9.93 & 0.0018 & 4862.02 & $4864.50 \pm 3.29$ & -9.66 & $-8.63 \pm 2.57$ \\
\hline & 160 & $\mathrm{C}_{28} \mathrm{H}_{14}$ & 199.09 & 201.73 & 0.52 & 11.87 & 0.0020 & 5672.07 & $5674.83 \pm 3.64$ & -11.27 & $-10.06 \pm 3.00$ \\
\hline & 161 & $\mathrm{C}_{32} \mathrm{H}_{16}$ & 227.10 & 229.73 & 0.63 & 13.53 & 0.0023 & 6481.90 & $6484.87 \pm 3.88$ & -12.88 & $-11.47 \pm 3.43$ \\
\hline
\end{tabular}

${ }^{a}$ All energies and enthalpies are reported in kcal/mol; molecules are identified by their molecule index MID as specified in Table S1. $\Delta_{\mathrm{B} 5 / / \mathrm{B} 3 \mathrm{LYP}}^{\mathrm{geo}}$ is defined in Sec 4.2, $\Delta H^{0}[T]$ is the thermal enthalpy difference between $T=0 \mathrm{~K}$ and $T=298.15 \mathrm{~K}$ (see Eq. (4)). $\Delta T_{1}\left(2_{s d}\right)$ reports the $T_{1}$ diagnostic, computed using a $2_{s d}$ basis set (cc-pVDZ without polarization functions on hydrogens), relative to the weighted average of $T_{1}$ values obtained for ethane, ethylene and acetylene. The weights represent the fraction of single, double, and triple bonds in the molecule. See Ref. 38 for details. Column "heat terms" reports all terms for the ATOMIC protocol that contribute to the sum $\Delta H_{f}^{0, A T O M I C}[298.15 K]+E_{A, e}^{\text {ATOMIC }}$ (see Eq. (3)). Corresponding values for ATOMIC(hc) include corrections $C^{\mathrm{ZPE}}, C^{\text {atoms }}$ and uncertainties $u^{\mathrm{ZPE}}, u^{\text {thermal }}, u^{\text {atoms }}$ as well (see Eqs. (9), (10); $C^{\text {thermal }}$ not included, see Ref. 138). Column "atomization energy terms" reports all terms for the ATOMIC protocol that contribute to the difference $E_{A, e}^{\mathrm{ATOMIC}}-E_{A, e}^{\mathrm{CCSD}(\mathrm{T})}$ (see Eq. (1)). Corresponding ATOMIC(hc) values additionally consider geometry corrections and uncertainties $\left(C^{\text {geo }}, u^{\text {geo }}\right)$ as well as all corrections $C$ and uncertainties $u$ defined in Ref. 38 except model-dependent low-level $\operatorname{CCSD}(\mathrm{T})$ terms $C_{\text {reac }}^{\mathrm{CCSD}(\mathrm{T})\{L\}}$ and $u_{\text {reac }}^{\mathrm{CCSD}(\mathrm{T})\{L\}}$. Data reported in columns "heat terms" and "atomization energy terms" supplement $\operatorname{CCSD}(\mathrm{T})$ atomization energies $E_{A, e}^{\mathrm{CCSD}(\mathrm{T})\{m\}}$ (Table S2) and low level CCSD(T) corrections (Table 1) to derive ATOMIC and ATOMIC(hc) heats of formation (Tables 3, S8 for the latter). 
Table S8: Enthalpies of formation $(298.15 \mathrm{~K} \text {, in } \mathrm{kcal} / \mathrm{mol})^{a}$

\begin{tabular}{|c|c|c|c|c|c|c|c|c|c|c|}
\hline \multirow{2}{*}{\multicolumn{2}{|c|}{ MID }} & \multicolumn{9}{|c|}{ ATOMIC(hc) } \\
\hline & & $\mathrm{A}$ & $\mathrm{B}_{1}$ & $\mathrm{~B}_{2}$ & $\mathrm{~B}_{3}$ & $\mathrm{~B}_{4}$ & $\mathrm{~B}_{5}$ & $\mathrm{~B}_{6}$ & $\mathrm{C}$ & $\mathrm{E}_{\mathrm{CCSD}}$ \\
\hline 1 & $\mathrm{CH}_{4}$ & $-17.7 \pm 0.5$ & $-17.6 \pm 0.5$ & $-17.8 \pm 0.5$ & $-17.6 \pm 0.5$ & $-17.5 \pm 0.6$ & $-17.6 \pm 0.6$ & $-17.6 \pm 0.7$ & $-17.3 \pm 1.2$ & $-17.6 \pm 0.7$ \\
\hline 2 & $\mathrm{C}_{2} \mathrm{H}_{2}$ & $54.7 \pm 0.6$ & $54.8 \pm 0.6$ & $54.7 \pm 0.6$ & $54.8 \pm 0.6$ & $55.0 \pm 0.7$ & $54.9 \pm 0.6$ & $54.9 \pm 0.8$ & $55.2 \pm 1.2$ & $54.8 \pm 0.7$ \\
\hline 3 & $\mathrm{C}_{2} \mathrm{H}_{4}$ & $12.6 \pm 0.5$ & $12.7 \pm 0.5$ & $12.5 \pm 0.6$ & $12.7 \pm 0.5$ & $12.9 \pm 0.7$ & $12.8 \pm 0.6$ & $12.7 \pm 0.9$ & $13.2 \pm 1.5$ & $12.7 \pm 0.8$ \\
\hline 4 & $\mathrm{C}_{2} \mathrm{H}_{6}$ & $-20.1 \pm 0.8$ & $-20.0 \pm 0.8$ & $-20.2 \pm 0.9$ & $-20.0 \pm 0.8$ & $-19.7 \pm 1.0$ & $-19.9 \pm 0.9$ & $-19.9 \pm 1.2$ & $-19.4 \pm 2.0$ & $-20.0 \pm 1.1$ \\
\hline 5 & $\mathrm{C}_{3} \mathrm{H}_{4}$ & $45.6 \pm 0.6$ & $45.4 \pm 0.7$ & $45.4 \pm 0.7$ & $45.4 \pm 0.7$ & $45.6 \pm 0.9$ & $45.5 \pm 0.8$ & $45.4 \pm 1.1$ & $45.8 \pm 1.9$ & $45.4 \pm 1.0$ \\
\hline 6 & $\mathrm{C}_{3} \mathrm{H}_{4}$ & $68.0 \pm 0.6$ & $68.1 \pm 0.6$ & $68.1 \pm 0.7$ & $68.2 \pm 0.6$ & $68.1 \pm 0.9$ & $68.0 \pm 0.7$ & $68.0 \pm 1.0$ & $68.1 \pm 1.8$ & $67.8 \pm 0.9$ \\
\hline 7 & $\mathrm{C}_{3} \mathrm{H}_{4}$ & $44.6 \pm 0.6$ & $44.5 \pm 0.7$ & $44.5 \pm 0.7$ & $44.5 \pm 0.7$ & $44.7 \pm 0.9$ & $44.6 \pm 0.8$ & $44.5 \pm 1.1$ & $45.2 \pm 1.9$ & $44.5 \pm 1.0$ \\
\hline 8 & $\mathrm{C}_{3} \mathrm{H}_{6}$ & $13.1 \pm 0.9$ & $13.4 \pm 0.9$ & $13.4 \pm 1.0$ & $13.6 \pm 0.9$ & $13.6 \pm 1.2$ & $13.4 \pm 1.0$ & $13.2 \pm 1.4$ & $13.3 \pm 2.3$ & $13.2 \pm 1.3$ \\
\hline 9 & $\mathrm{C}_{3} \mathrm{H}_{6}$ & $4.8 \pm 0.8$ & $4.9 \pm 0.8$ & $4.8 \pm 0.9$ & $4.9 \pm 0.8$ & $5.2 \pm 1.1$ & $5.0 \pm 0.9$ & $4.9 \pm 1.3$ & $5.7 \pm 2.3$ & $4.9 \pm 1.2$ \\
\hline 10 & $\mathrm{C}_{3} \mathrm{H}_{8}$ & $-25.2 \pm 1.1$ & $-25.1 \pm 1.1$ & $-25.3 \pm 1.2$ & $-25.0 \pm 1.1$ & $-24.7 \pm 1.4$ & $-24.9 \pm 1.2$ & $-25.0 \pm 1.7$ & $-24.1 \pm 2.7$ & $-25.0 \pm 1.5$ \\
\hline 11 & $\mathrm{C}_{4} \mathrm{H}_{2}$ & $110.6 \pm 1.1$ & $110.3 \pm 1.1$ & $110.3 \pm 1.1$ & $110.2 \pm 1.1$ & $110.4 \pm 1.3$ & $110.6 \pm 1.2$ & $110.9 \pm 1.4$ & $110.2 \pm 2.1$ & $110.4 \pm 1.3$ \\
\hline 12 & $\mathrm{C}_{4} \mathrm{H}_{4}$ & $137.0 \pm 0.9$ & $137.6 \pm 0.9$ & $137.3 \pm 1.0$ & $137.1 \pm 0.9$ & $136.3 \pm 1.1$ & $136.5 \pm 1.0$ & $136.3 \pm 1.3$ & $136.2 \pm 2.2$ & $137.5 \pm 1.2$ \\
\hline 13 & $\mathrm{C}_{4} \mathrm{H}_{4}$ & $77.7 \pm 0.9$ & $77.2 \pm 0.9$ & $77.3 \pm 1.0$ & $77.3 \pm 0.9$ & $77.4 \pm 1.2$ & $77.5 \pm 1.0$ & $77.6 \pm 1.4$ & $77.4 \pm 2.3$ & $77.9 \pm 1.3$ \\
\hline 14 & $\mathrm{C}_{4} \mathrm{H}_{4}$ & $102.8 \pm 0.9$ & $103.2 \pm 0.9$ & $102.9 \pm 0.9$ & $103.1 \pm 0.9$ & $102.8 \pm 1.1$ & $102.9 \pm 1.0$ & $103.0 \pm 1.3$ & $103.1 \pm 2.2$ & $104.1 \pm 1.2$ \\
\hline 15 & $\mathrm{C}_{4} \mathrm{H}_{4}$ & $69.7 \pm 0.9$ & $69.5 \pm 1.0$ & $69.5 \pm 1.0$ & $69.5 \pm 1.0$ & $69.7 \pm 1.2$ & $69.7 \pm 1.1$ & $69.8 \pm 1.4$ & $70.0 \pm 2.3$ & $69.7 \pm 1.3$ \\
\hline 16 & $\mathrm{C}_{4} \mathrm{H}_{4}$ & $92.9 \pm 0.8$ & $92.9 \pm 0.8$ & $93.0 \pm 0.9$ & $93.0 \pm 0.8$ & $93.0 \pm 1.1$ & $93.1 \pm 0.9$ & $92.8 \pm 1.3$ & $93.4 \pm 2.2$ & $92.7 \pm 1.2$ \\
\hline 17 & $\mathrm{C}_{4} \mathrm{H}_{4}$ & $129.0 \pm 0.8$ & $129.6 \pm 0.8$ & $129.9 \pm 0.9$ & $129.5 \pm 0.8$ & $128.9 \pm 1.1$ & $128.8 \pm 0.9$ & $128.4 \pm 1.3$ & $127.3 \pm 2.1$ & $128.9 \pm 1.2$ \\
\hline 18 & $\mathrm{C}_{4} \mathrm{H}_{6}$ & $39.1 \pm 0.9$ & $38.9 \pm 0.9$ & $38.9 \pm 1.0$ & $38.9 \pm 0.9$ & $39.2 \pm 1.3$ & $39.0 \pm 1.1$ & $38.9 \pm 1.6$ & $39.5 \pm 2.7$ & $38.9 \pm 1.4$ \\
\hline 19 & $\mathrm{C}_{4} \mathrm{H}_{6}$ & $26.9 \pm 0.9$ & $26.9 \pm 1.0$ & $26.8 \pm 1.1$ & $26.9 \pm 1.0$ & $27.2 \pm 1.3$ & $27.1 \pm 1.1$ & $27.1 \pm 1.6$ & $27.6 \pm 2.7$ & $27.1 \pm 1.4$ \\
\hline 20 & $\mathrm{C}_{4} \mathrm{H}_{6}$ & $35.4 \pm 0.8$ & $35.2 \pm 0.9$ & $35.3 \pm 1.0$ & $35.1 \pm 0.9$ & $35.4 \pm 1.3$ & $35.3 \pm 1.0$ & $35.3 \pm 1.5$ & $36.2 \pm 2.7$ & $35.1 \pm 1.4$ \\
\hline 21 & $\mathrm{C}_{4} \mathrm{H}_{6}$ & $54.0 \pm 0.9$ & $54.4 \pm 0.9$ & $54.4 \pm 1.0$ & $54.4 \pm 0.9$ & $54.1 \pm 1.3$ & $54.0 \pm 1.1$ & $53.8 \pm 1.5$ & $53.6 \pm 2.6$ & $53.9 \pm 1.4$ \\
\hline 22 & $\mathrm{C}_{4} \mathrm{H}_{6}$ & $38.6 \pm 0.8$ & $38.7 \pm 0.9$ & $38.6 \pm 1.0$ & $38.8 \pm 0.9$ & $38.8 \pm 1.2$ & $38.7 \pm 1.0$ & $38.8 \pm 1.5$ & $39.4 \pm 2.7$ & $38.6 \pm 1.4$ \\
\hline 23 & $\mathrm{C}_{4} \mathrm{H}_{6}$ & $46.4 \pm 0.9$ & $46.5 \pm 0.9$ & $46.7 \pm 1.0$ & $46.6 \pm 0.9$ & $46.7 \pm 1.3$ & $46.5 \pm 1.1$ & $46.4 \pm 1.5$ & $46.8 \pm 2.6$ & $46.6 \pm 1.4$ \\
\hline 24 & $\mathrm{C}_{4} \mathrm{H}_{8}$ & $6.5 \pm 1.2$ & $6.7 \pm 1.2$ & $6.4 \pm 1.3$ & $6.8 \pm 1.2$ & $6.9 \pm 1.6$ & $6.7 \pm 1.4$ & $6.7 \pm 1.9$ & $7.2 \pm 3.1$ & $6.7 \pm 1.7$ \\
\hline 25 & $\mathrm{C}_{4} \mathrm{H}_{8}$ & $-3.9 \pm 1.0$ & $-3.8 \pm 1.1$ & $-3.9 \pm 1.2$ & $-3.8 \pm 1.1$ & $-3.5 \pm 1.5$ & $-3.8 \pm 1.3$ & $-3.9 \pm 1.8$ & $-2.9 \pm 3.1$ & $-3.8 \pm 1.6$ \\
\hline 26 & $\mathrm{C}_{4} \mathrm{H}_{10}$ & $-32.0 \pm 1.3$ & $-32.0 \pm 1.4$ & $-32.2 \pm 1.4$ & $-32.0 \pm 1.3$ & $-31.7 \pm 1.8$ & $-32.0 \pm 1.5$ & $-32.1 \pm 2.1$ & $-30.9 \pm 3.5$ & $-31.9 \pm 1.9$ \\
\hline 27 & $\mathrm{C}_{4} \mathrm{H}_{10}$ & $-29.8 \pm 1.3$ & $-29.8 \pm 1.4$ & $-30.1 \pm 1.5$ & $-29.7 \pm 1.4$ & $-29.4 \pm 1.8$ & $-29.7 \pm 1.6$ & $-29.8 \pm 2.1$ & $-28.6 \pm 3.5$ & $-29.7 \pm 1.9$ \\
\hline 28 & $\mathrm{C}_{5} \mathrm{H}_{4}$ & $109.3 \pm 1.1$ & $109.0 \pm 1.1$ & $109.0 \pm 1.2$ & $108.8 \pm 1.1$ & $109.1 \pm 1.4$ & $109.1 \pm 1.2$ & $109.0 \pm 1.6$ & $109.6 \pm 2.7$ & $108.9 \pm 1.5$ \\
\hline
\end{tabular}


Table S8, contd.

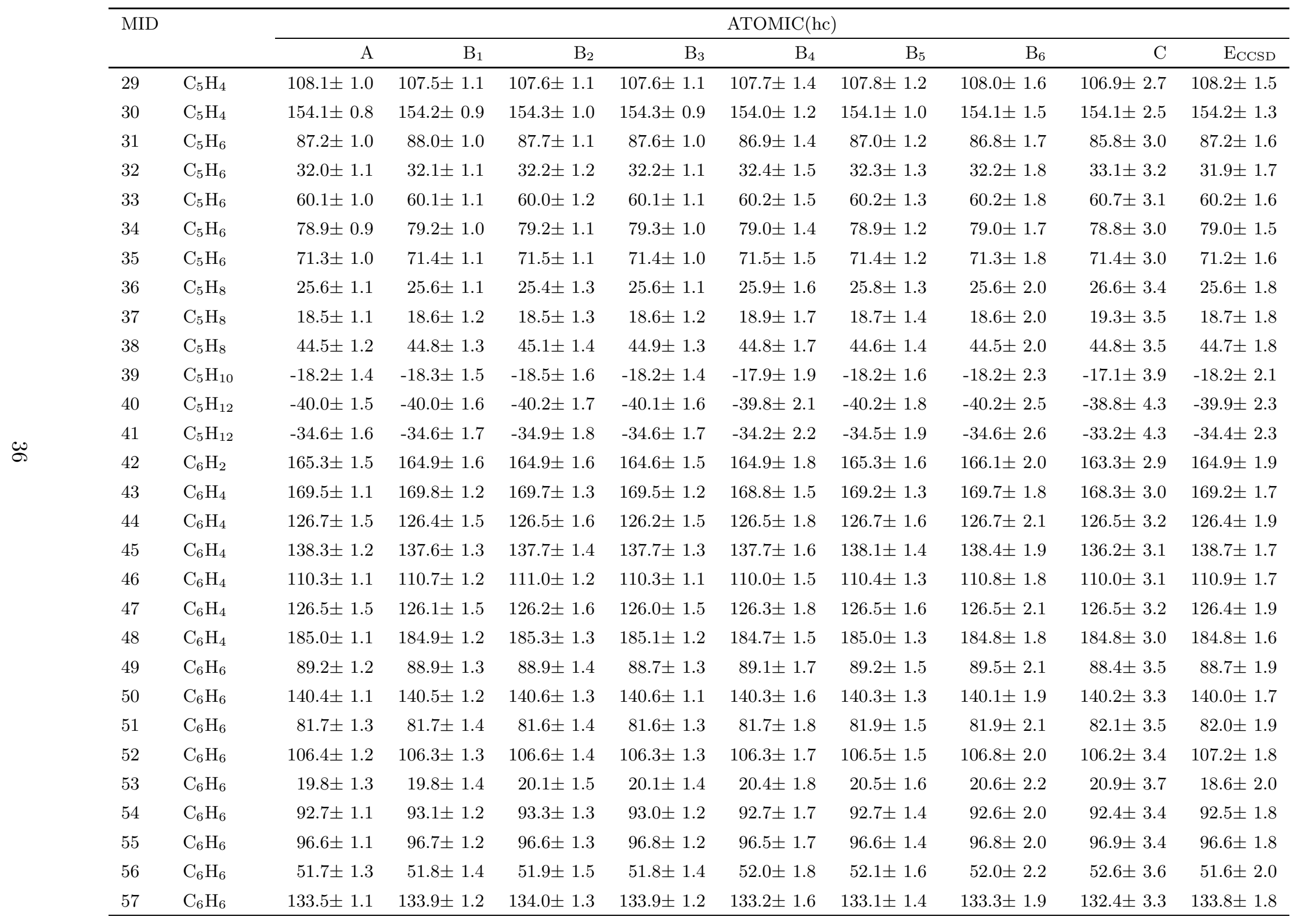


Table S8, contd.

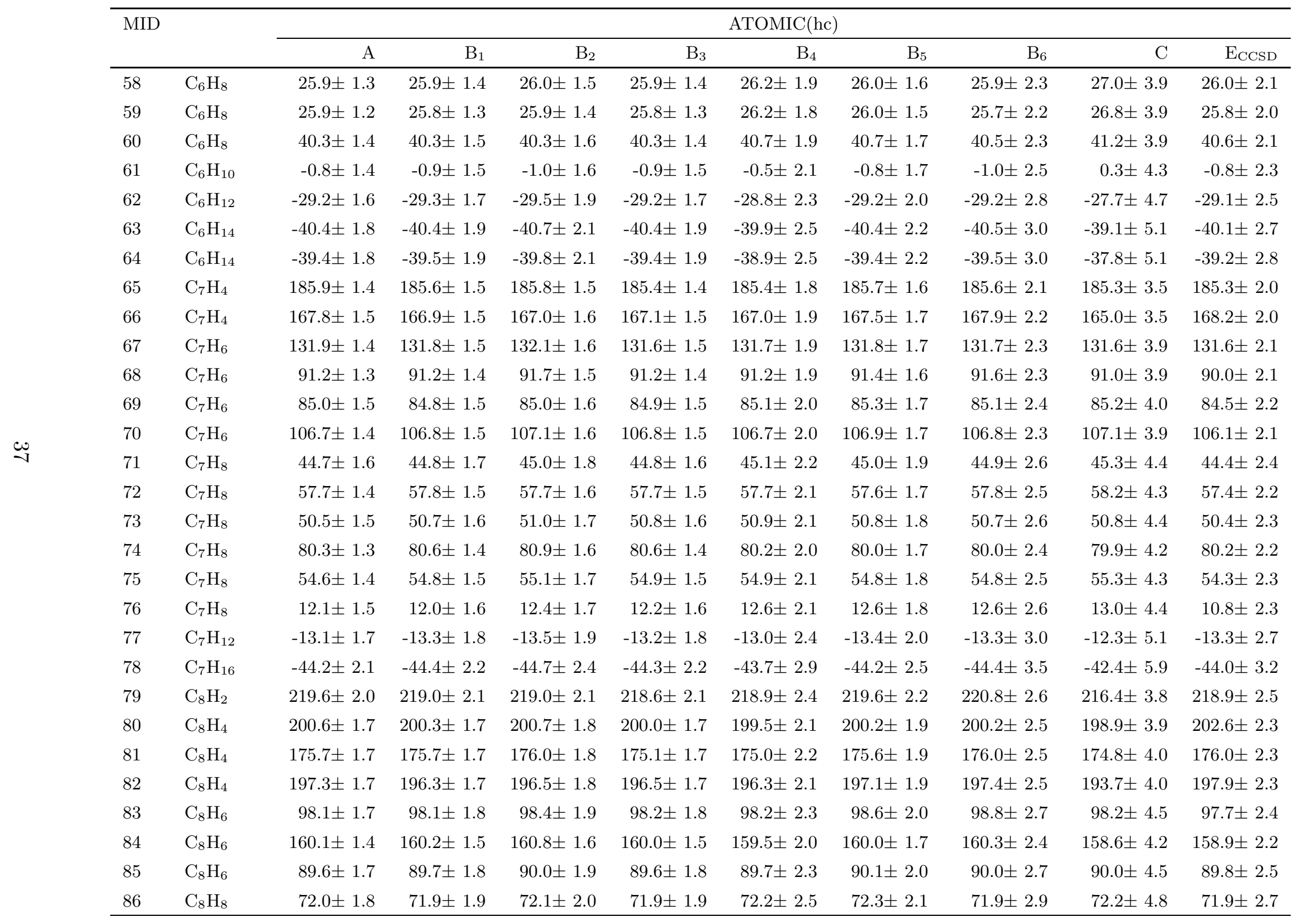


Table S8, contd.

\begin{tabular}{|c|c|c|c|c|c|c|c|c|c|c|}
\hline \multirow[t]{2}{*}{ MID } & & \multicolumn{9}{|c|}{ ATOMIC(hc) } \\
\hline & & $\mathrm{A}$ & $\mathrm{B}_{1}$ & $\mathrm{~B}_{2}$ & $\mathrm{~B}_{3}$ & $\mathrm{~B}_{4}$ & $\mathrm{~B}_{5}$ & $\mathrm{~B}_{6}$ & $\mathrm{C}$ & $\mathrm{E}_{\mathrm{CCSD}}$ \\
\hline 87 & $\mathrm{C}_{8} \mathrm{H}_{8}$ & $47.9 \pm 1.6$ & $47.9 \pm 1.7$ & $48.2 \pm 1.9$ & $48.0 \pm 1.7$ & $48.2 \pm 2.3$ & $48.4 \pm 2.0$ & $48.4 \pm 2.8$ & $48.4 \pm 4.8$ & $46.7 \pm 2.5$ \\
\hline 88 & $\mathrm{C}_{8} \mathrm{H}_{8}$ & $145.4 \pm 1.6$ & $145.9 \pm 1.7$ & $145.6 \pm 1.8$ & $146.0 \pm 1.7$ & $145.3 \pm 2.3$ & $145.2 \pm 1.9$ & $145.8 \pm 2.7$ & $144.5 \pm 4.6$ & $146.7 \pm 2.5$ \\
\hline 89 & $\mathrm{C}_{8} \mathrm{H}_{8}$ & $53.6 \pm 1.9$ & $53.6 \pm 2.0$ & $53.9 \pm 2.1$ & $53.4 \pm 1.9$ & $53.8 \pm 2.5$ & $54.0 \pm 2.2$ & $53.7 \pm 3.0$ & $53.8 \pm 4.9$ & $54.2 \pm 2.7$ \\
\hline 90 & $\mathrm{C}_{8} \mathrm{H}_{8}$ & $35.9 \pm 1.7$ & $35.8 \pm 1.8$ & $36.2 \pm 2.0$ & $36.0 \pm 1.8$ & $36.4 \pm 2.4$ & $36.6 \pm 2.1$ & $36.5 \pm 2.9$ & $36.5 \pm 4.9$ & $34.7 \pm 2.6$ \\
\hline 91 & $\mathrm{C}_{8} \mathrm{H}_{10}$ & $53.6 \pm 1.9$ & $53.5 \pm 2.0$ & $53.5 \pm 2.1$ & $53.5 \pm 1.9$ & $54.0 \pm 2.6$ & $53.9 \pm 2.2$ & $53.7 \pm 3.1$ & $54.5 \pm 5.2$ & $53.9 \pm 2.8$ \\
\hline 92 & $\mathrm{C}_{8} \mathrm{H}_{14}$ & $-22.6 \pm 2.0$ & $-22.9 \pm 2.1$ & $-23.1 \pm 2.3$ & $-22.8 \pm 2.1$ & $-22.4 \pm 2.8$ & $-22.9 \pm 2.4$ & $-22.9 \pm 3.4$ & $-21.6 \pm 5.9$ & $-22.7 \pm 3.1$ \\
\hline 93 & $\mathrm{C}_{8} \mathrm{H}_{18}$ & $-49.1 \pm 2.4$ & $-49.2 \pm 2.5$ & $-49.6 \pm 2.7$ & $-49.1 \pm 2.5$ & $-48.5 \pm 3.3$ & $-49.1 \pm 2.8$ & $-49.3 \pm 3.9$ & $-47.0 \pm 6.7$ & $-48.8 \pm 3.6$ \\
\hline 94 & $\mathrm{C}_{9} \mathrm{H}_{20}$ & $-53.9 \pm 2.6$ & $-54.1 \pm 2.8$ & $-54.5 \pm 3.0$ & $-54.0 \pm 2.8$ & $-53.3 \pm 3.7$ & $-54.0 \pm 3.2$ & $-54.2 \pm 4.4$ & $-51.7 \pm 7.5$ & $-53.6 \pm 4.0$ \\
\hline 95 & $\mathrm{C}_{10} \mathrm{H}_{2}$ & $273.8 \pm 2.6$ & $273.0 \pm 2.6$ & $273.1 \pm 2.7$ & $272.5 \pm 2.6$ & $272.9 \pm 3.0$ & $273.8 \pm 2.8$ & $275.5 \pm 3.3$ & $269.2 \pm 4.7$ & $272.8 \pm 3.1$ \\
\hline 96 & $\mathrm{C}_{10} \mathrm{H}_{4}$ & $245.3 \pm 2.5$ & $244.8 \pm 2.5$ & $245.1 \pm 2.6$ & $244.3 \pm 2.5$ & $244.6 \pm 3.0$ & $245.2 \pm 2.7$ & $245.1 \pm 3.3$ & $243.0 \pm 5.0$ & $244.3 \pm 3.1$ \\
\hline 97 & $\mathrm{C}_{10} \mathrm{H}_{8}$ & $71.8 \pm 1.9$ & $71.8 \pm 2.0$ & $72.3 \pm 2.2$ & $71.7 \pm 2.0$ & $72.2 \pm 2.7$ & $72.6 \pm 2.3$ & $72.2 \pm 3.3$ & $71.6 \pm 5.7$ & $70.6 \pm 3.0$ \\
\hline 98 & $\mathrm{C}_{10} \mathrm{H}_{8}$ & $35.5 \pm 2.2$ & $35.5 \pm 2.3$ & $36.2 \pm 2.4$ & $35.6 \pm 2.3$ & $36.2 \pm 3.0$ & $36.6 \pm 2.6$ & $36.4 \pm 3.5$ & $35.8 \pm 5.9$ & $33.6 \pm 3.2$ \\
\hline 99 & $\mathrm{C}_{10} \mathrm{H}_{8}$ & $89.3 \pm 2.2$ & $89.4 \pm 2.3$ & $89.8 \pm 2.5$ & $89.4 \pm 2.3$ & $89.7 \pm 3.0$ & $90.1 \pm 2.6$ & $89.8 \pm 3.5$ & $89.9 \pm 5.8$ & $89.0 \pm 3.2$ \\
\hline 100 & $\mathrm{C}_{10} \mathrm{H}_{10}$ & $141.7 \pm 2.0$ & $141.7 \pm 2.1$ & $142.1 \pm 2.2$ & $141.6 \pm 2.1$ & $141.8 \pm 2.8$ & $141.9 \pm 2.4$ & $142.1 \pm 3.4$ & $139.7 \pm 5.8$ & $141.4 \pm 3.1$ \\
\hline 101 & $\mathrm{C}_{10} \mathrm{H}_{10}$ & $62.2 \pm 2.1$ & $62.0 \pm 2.2$ & $62.4 \pm 2.4$ & $62.0 \pm 2.2$ & $62.6 \pm 2.9$ & $62.9 \pm 2.5$ & $62.8 \pm 3.5$ & $62.4 \pm 6.0$ & $60.8 \pm 3.2$ \\
\hline 102 & $\mathrm{C}_{10} \mathrm{H}_{10}$ & $71.0 \pm 2.0$ & $70.9 \pm 2.1$ & $71.6 \pm 2.3$ & $71.1 \pm 2.1$ & $71.4 \pm 2.9$ & $71.5 \pm 2.4$ & $71.4 \pm 3.5$ & $71.2 \pm 6.0$ & $69.9 \pm 3.1$ \\
\hline 103 & $\mathrm{C}_{10} \mathrm{H}_{10}$ & $87.1 \pm 2.1$ & $86.9 \pm 2.2$ & $87.3 \pm 2.4$ & $86.8 \pm 2.2$ & $87.0 \pm 3.0$ & $87.2 \pm 2.5$ & $86.9 \pm 3.5$ & $86.6 \pm 6.0$ & $87.7 \pm 3.2$ \\
\hline 104 & $\mathrm{C}_{10} \mathrm{H}_{10}$ & $109.9 \pm 2.0$ & $109.9 \pm 2.1$ & $109.9 \pm 2.3$ & $109.9 \pm 2.1$ & $109.5 \pm 2.8$ & $109.5 \pm 2.4$ & $109.8 \pm 3.4$ & $109.0 \pm 5.9$ & $110.2 \pm 3.1$ \\
\hline 105 & $\mathrm{C}_{10} \mathrm{H}_{10}$ & $74.2 \pm 1.9$ & $74.1 \pm 2.1$ & $74.5 \pm 2.2$ & $74.1 \pm 2.0$ & $74.2 \pm 2.8$ & $74.4 \pm 2.4$ & $74.5 \pm 3.4$ & $74.1 \pm 6.0$ & $73.0 \pm 3.1$ \\
\hline 106 & $\mathrm{C}_{10} \mathrm{H}_{10}$ & $82.1 \pm 2.0$ & $82.0 \pm 2.1$ & $82.6 \pm 2.3$ & $81.9 \pm 2.1$ & $82.0 \pm 2.9$ & $82.0 \pm 2.4$ & $81.8 \pm 3.5$ & $81.8 \pm 6.0$ & $81.9 \pm 3.1$ \\
\hline 107 & $\mathrm{C}_{10} \mathrm{H}_{10}$ & $91.2 \pm 1.9$ & $91.4 \pm 2.0$ & $92.1 \pm 2.2$ & $91.3 \pm 2.0$ & $90.8 \pm 2.8$ & $90.7 \pm 2.4$ & $90.6 \pm 3.4$ & $90.2 \pm 5.9$ & $91.1 \pm 3.1$ \\
\hline 108 & $\mathrm{C}_{10} \mathrm{H}_{10}$ & $121.1 \pm 2.2$ & $120.7 \pm 2.3$ & $120.8 \pm 2.4$ & $120.4 \pm 2.3$ & $120.9 \pm 3.0$ & $121.2 \pm 2.6$ & $121.4 \pm 3.5$ & $119.5 \pm 5.9$ & $120.7 \pm 3.2$ \\
\hline 109 & $\mathrm{C}_{10} \mathrm{H}_{10}$ & $89.4 \pm 2.0$ & $89.3 \pm 2.2$ & $89.5 \pm 2.3$ & $89.2 \pm 2.1$ & $89.1 \pm 2.9$ & $89.1 \pm 2.5$ & $89.2 \pm 3.5$ & $89.2 \pm 6.0$ & $88.7 \pm 3.2$ \\
\hline 110 & $\mathrm{C}_{10} \mathrm{H}_{10}$ & $74.4 \pm 1.9$ & $74.5 \pm 2.1$ & $75.1 \pm 2.2$ & $74.4 \pm 2.0$ & $74.0 \pm 2.8$ & $73.9 \pm 2.4$ & $73.9 \pm 3.4$ & $73.8 \pm 6.0$ & $73.9 \pm 3.1$ \\
\hline 111 & $\mathrm{C}_{10} \mathrm{H}_{10}$ & $58.9 \pm 2.0$ & $58.5 \pm 2.1$ & $58.8 \pm 2.3$ & $58.4 \pm 2.1$ & $58.6 \pm 2.9$ & $58.6 \pm 2.4$ & $58.3 \pm 3.5$ & $59.3 \pm 6.0$ & $58.6 \pm 3.1$ \\
\hline 112 & $\mathrm{C}_{10} \mathrm{H}_{12}$ & $66.7 \pm 2.3$ & $66.6 \pm 2.4$ & $66.6 \pm 2.6$ & $66.6 \pm 2.4$ & $67.1 \pm 3.2$ & $67.2 \pm 2.8$ & $66.8 \pm 3.8$ & $67.7 \pm 6.4$ & $67.1 \pm 3.5$ \\
\hline 113 & $\mathrm{C}_{10} \mathrm{H}_{16}$ & $-32.1 \pm 2.3$ & $-32.7 \pm 2.4$ & $-32.7 \pm 2.6$ & $-32.7 \pm 2.4$ & $-32.3 \pm 3.4$ & $-32.9 \pm 2.8$ & $-32.9 \pm 4.1$ & $-31.2 \pm 7.2$ & $-32.5 \pm 3.7$ \\
\hline 114 & $\mathrm{C}_{10} \mathrm{H}_{16}$ & $-22.8 \pm 2.3$ & $-23.4 \pm 2.5$ & $-23.5 \pm 2.7$ & $-23.3 \pm 2.4$ & $-22.9 \pm 3.4$ & $-23.5 \pm 2.8$ & $-23.5 \pm 4.1$ & $-22.2 \pm 7.1$ & $-23.0 \pm 3.7$ \\
\hline 115 & $\mathrm{C}_{10} \mathrm{H}_{18}$ & $-40.5 \pm 2.5$ & $-41.2 \pm 2.6$ & $-41.3 \pm 2.8$ & $-41.0 \pm 2.6$ & $-40.5 \pm 3.6$ & $-41.2 \pm 3.0$ & $-41.3 \pm 4.4$ & $-39.2 \pm 7.5$ & $-40.6 \pm 3.9$ \\
\hline
\end{tabular}


Table S8, contd.

\begin{tabular}{|c|c|c|c|c|c|c|c|c|c|c|}
\hline \multirow[t]{2}{*}{ MID } & & \multicolumn{9}{|c|}{ ATOMIC(hc) } \\
\hline & & $\mathrm{A}$ & $\mathrm{B}_{1}$ & $\mathrm{~B}_{2}$ & $\mathrm{~B}_{3}$ & $\mathrm{~B}_{4}$ & $\mathrm{~B}_{5}$ & $\mathrm{~B}_{6}$ & $\mathrm{C}$ & $\mathrm{E}_{\mathrm{CCSD}}$ \\
\hline 116 & $\mathrm{C}_{10} \mathrm{H}_{18}$ & $-43.5 \pm 2.4$ & $-44.0 \pm 2.6$ & $-44.1 \pm 2.8$ & $-43.9 \pm 2.6$ & $-43.4 \pm 3.6$ & $-44.0 \pm 3.0$ & $-44.2 \pm 4.3$ & $-41.9 \pm 7.5$ & $-43.5 \pm 3.9$ \\
\hline 117 & $\mathrm{C}_{10} \mathrm{H}_{22}$ & $-58.7 \pm 2.9$ & $-59.0 \pm 3.1$ & $-59.5 \pm 3.3$ & $-58.9 \pm 3.1$ & $-58.1 \pm 4.1$ & $-58.8 \pm 3.5$ & $-59.2 \pm 4.9$ & $-56.3 \pm 8.3$ & $-58.4 \pm 4.4$ \\
\hline 118 & $\mathrm{C}_{11} \mathrm{H}_{24}$ & $-63.5 \pm 3.2$ & $-63.9 \pm 3.4$ & $-64.4 \pm 3.6$ & $-63.7 \pm 3.3$ & $-62.9 \pm 4.5$ & $-63.7 \pm 3.8$ & $-64.1 \pm 5.3$ & $-61.0 \pm 9.0$ & $-63.2 \pm 4.8$ \\
\hline 119 & $\mathrm{C}_{12} \mathrm{H}_{6}$ & $256.1 \pm 2.4$ & $256.7 \pm 2.5$ & $257.3 \pm 2.6$ & $255.8 \pm 2.5$ & $254.7 \pm 3.1$ & $255.9 \pm 2.7$ & $256.4 \pm 3.6$ & $253.7 \pm 6.0$ & $257.4 \pm 3.3$ \\
\hline 120 & $\mathrm{C}_{12} \mathrm{H}_{6}$ & $355.7 \pm 2.2$ & $355.5 \pm 2.3$ & $356.4 \pm 2.4$ & $355.7 \pm 2.3$ & $354.9 \pm 2.9$ & $356.0 \pm 2.6$ & $355.6 \pm 3.4$ & $354.0 \pm 5.7$ & $355.3 \pm 3.1$ \\
\hline 121 & $\mathrm{C}_{12} \mathrm{H}_{8}$ & $62.7 \pm 2.6$ & $62.7 \pm 2.7$ & $63.6 \pm 2.9$ & $62.7 \pm 2.7$ & $63.2 \pm 3.5$ & $63.9 \pm 3.0$ & $63.7 \pm 4.1$ & $62.3 \pm 6.8$ & $60.6 \pm 3.7$ \\
\hline 122 & $\mathrm{C}_{12} \mathrm{H}_{8}$ & $100.2 \pm 2.5$ & $100.1 \pm 2.6$ & $100.8 \pm 2.8$ & $100.3 \pm 2.6$ & $100.5 \pm 3.4$ & $101.3 \pm 2.9$ & $101.5 \pm 4.0$ & $99.6 \pm 6.6$ & $98.2 \pm 3.6$ \\
\hline 123 & $\mathrm{C}_{12} \mathrm{H}_{10}$ & $42.9 \pm 2.5$ & $42.8 \pm 2.7$ & $43.6 \pm 2.8$ & $43.1 \pm 2.6$ & $43.7 \pm 3.5$ & $44.1 \pm 3.0$ & $44.0 \pm 4.2$ & $43.6 \pm 7.1$ & $40.4 \pm 3.8$ \\
\hline 124 & $\mathrm{C}_{12} \mathrm{H}_{12}$ & & & & $54.6 \pm 2.6$ & $55.1 \pm 3.6$ & $55.3 \pm 3.0$ & $54.9 \pm 4.3$ & $54.8 \pm 7.3$ & $53.4 \pm 3.9$ \\
\hline 125 & $\mathrm{C}_{12} \mathrm{H}_{14}$ & $79.8 \pm 2.8$ & $79.6 \pm 2.9$ & $79.7 \pm 3.1$ & $79.6 \pm 2.9$ & $80.3 \pm 3.8$ & $80.3 \pm 3.3$ & $79.8 \pm 4.5$ & $80.8 \pm 7.6$ & $80.3 \pm 4.1$ \\
\hline 126 & $\mathrm{C}_{12} \mathrm{H}_{18}$ & $-17.6 \pm 2.7$ & $-17.6 \pm 2.9$ & $-17.0 \pm 3.1$ & $-17.6 \pm 2.8$ & $-17.0 \pm 4.0$ & $-17.3 \pm 3.3$ & $-17.9 \pm 4.8$ & $-17.5 \pm 8.3$ & $-18.6 \pm 4.3$ \\
\hline 127 & $\mathrm{C}_{12} \mathrm{H}_{26}$ & $-68.3 \pm 3.5$ & $-68.8 \pm 3.7$ & $-69.3 \pm 3.9$ & $-68.6 \pm 3.6$ & $-67.7 \pm 4.9$ & $-68.6 \pm 4.2$ & $-69.0 \pm 5.8$ & $-65.6 \pm 9.8$ & $-67.9 \pm 5.3$ \\
\hline 128 & $\mathrm{C}_{13} \mathrm{H}_{10}$ & $45.0 \pm 2.7$ & $44.8 \pm 2.8$ & $45.8 \pm 3.0$ & $45.0 \pm 2.8$ & $45.6 \pm 3.7$ & $46.1 \pm 3.2$ & $45.9 \pm 4.4$ & $45.1 \pm 7.5$ & $42.2 \pm 4.0$ \\
\hline 129 & $\mathrm{C}_{13} \mathrm{H}_{28}$ & $-73.1 \pm 3.7$ & $-73.6 \pm 3.9$ & $-74.2 \pm 4.2$ & $-73.4 \pm 3.9$ & $-72.5 \pm 5.2$ & $-73.4 \pm 4.4$ & $-73.9 \pm 6.2$ & $-70.2 \pm 10.6$ & $-72.7 \pm 5.7$ \\
\hline 130 & $\mathrm{C}_{14} \mathrm{H}_{8}$ & $137.4 \pm 2.6$ & $137.8 \pm 2.8$ & $138.8 \pm 3.0$ & $137.4 \pm 2.7$ & $137.5 \pm 3.7$ & $138.6 \pm 3.1$ & $138.6 \pm 4.4$ & $135.7 \pm 7.4$ & $135.9 \pm 4.0$ \\
\hline 131 & $\mathrm{C}_{14} \mathrm{H}_{8}$ & $103.3 \pm 3.0$ & $103.4 \pm 3.1$ & $104.5 \pm 3.3$ & $103.3 \pm 3.1$ & $103.6 \pm 4.0$ & $104.7 \pm 3.5$ & $104.5 \pm 4.6$ & $101.9 \pm 7.6$ & $101.5 \pm 4.3$ \\
\hline 132 & $\mathrm{C}_{14} \mathrm{H}_{10}$ & $54.8 \pm 3.1$ & $54.7 \pm 3.2$ & $55.8 \pm 3.4$ & $54.8 \pm 3.2$ & $55.5 \pm 4.1$ & $56.3 \pm 3.6$ & $55.8 \pm 4.9$ & $54.2 \pm 8.1$ & $52.4 \pm 4.5$ \\
\hline 133 & $\mathrm{C}_{14} \mathrm{H}_{10}$ & $49.0 \pm 3.1$ & $49.0 \pm 3.2$ & $50.1 \pm 3.4$ & $49.2 \pm 3.2$ & $49.9 \pm 4.1$ & $50.7 \pm 3.6$ & $50.3 \pm 4.9$ & $48.5 \pm 8.1$ & $46.2 \pm 4.5$ \\
\hline 134 & $\mathrm{C}_{14} \mathrm{H}_{20}$ & $-37.8 \pm 3.0$ & $-39.0 \pm 3.2$ & $-38.8 \pm 3.5$ & $-39.1 \pm 3.2$ & $-38.7 \pm 4.5$ & $-39.3 \pm 3.7$ & $-39.4 \pm 5.5$ & $-37.6 \pm 9.6$ & $-38.6 \pm 4.9$ \\
\hline 135 & $\mathrm{C}_{14} \mathrm{H}_{30}$ & $-77.9 \pm 4.0$ & $-78.5 \pm 4.2$ & $-79.1 \pm 4.5$ & $-78.3 \pm 4.2$ & $-77.3 \pm 5.6$ & $-78.3 \pm 4.8$ & $-78.8 \pm 6.7$ & $-74.9 \pm 11.4$ & $-77.5 \pm 6.1$ \\
\hline 136 & $\mathrm{C}_{15} \mathrm{H}_{32}$ & $-82.8 \pm 4.2$ & $-83.4 \pm 4.5$ & $-84.0 \pm 4.8$ & $-83.2 \pm 4.4$ & $-82.1 \pm 6.0$ & $-83.1 \pm 5.1$ & $-83.7 \pm 7.2$ & $-79.5 \pm 12.2$ & $-82.3 \pm 6.5$ \\
\hline 137 & $\mathrm{C}_{16} \mathrm{H}_{8}$ & $195.2 \pm 3.2$ & $195.2 \pm 3.3$ & $196.4 \pm 3.5$ & $194.7 \pm 3.3$ & $194.4 \pm 4.3$ & $195.8 \pm 3.7$ & $196.3 \pm 5.0$ & $193.2 \pm 8.3$ & $193.8 \pm 4.6$ \\
\hline 138 & $\mathrm{C}_{16} \mathrm{H}_{10}$ & $68.2 \pm 3.4$ & $68.1 \pm 3.6$ & $69.4 \pm 3.8$ & $68.1 \pm 3.5$ & $68.8 \pm 4.6$ & $69.9 \pm 4.0$ & $69.6 \pm 5.4$ & $67.1 \pm 9.0$ & $64.7 \pm 4.9$ \\
\hline 139 & $\mathrm{C}_{16} \mathrm{H}_{10}$ & $53.9 \pm 3.5$ & $54.0 \pm 3.6$ & $55.4 \pm 3.9$ & $54.0 \pm 3.6$ & $54.8 \pm 4.6$ & $55.9 \pm 4.0$ & $55.3 \pm 5.5$ & $52.4 \pm 9.0$ & $50.8 \pm 5.0$ \\
\hline 140 & $\mathrm{C}_{16} \mathrm{H}_{12}$ & $123.8 \pm 3.4$ & $123.7 \pm 3.5$ & $124.7 \pm 3.8$ & $123.3 \pm 3.5$ & $123.4 \pm 4.6$ & $124.0 \pm 4.0$ & $123.6 \pm 5.4$ & $121.7 \pm 9.1$ & $120.8 \pm 5.0$ \\
\hline 141 & $\mathrm{C}_{16} \mathrm{H}_{16}$ & $41.1 \pm 3.4$ & $40.7 \pm 3.6$ & $41.5 \pm 3.8$ & $40.5 \pm 3.5$ & $41.0 \pm 4.8$ & $41.2 \pm 4.1$ & $41.0 \pm 5.7$ & $40.0 \pm 9.8$ & $37.7 \pm 5.2$ \\
\hline 142 & $\mathrm{C}_{16} \mathrm{H}_{16}$ & $48.8 \pm 3.3$ & $48.5 \pm 3.5$ & $49.4 \pm 3.8$ & $48.2 \pm 3.5$ & $48.6 \pm 4.7$ & $48.7 \pm 4.0$ & $48.5 \pm 5.7$ & $46.8 \pm 9.8$ & $45.2 \pm 5.2$ \\
\hline 143 & $\mathrm{C}_{16} \mathrm{H}_{16}$ & $59.7 \pm 3.4$ & $59.5 \pm 3.6$ & $60.3 \pm 3.9$ & $59.2 \pm 3.6$ & $59.5 \pm 4.8$ & $59.7 \pm 4.1$ & $59.4 \pm 5.7$ & $57.8 \pm 9.8$ & $56.5 \pm 5.2$ \\
\hline 144 & $\mathrm{C}_{16} \mathrm{H}_{34}$ & $-87.6 \pm 4.5$ & $-88.3 \pm 4.8$ & $-88.9 \pm 5.1$ & $-88.0 \pm 4.7$ & $-86.9 \pm 6.4$ & $-88.0 \pm 5.4$ & $-88.6 \pm 7.6$ & $-84.1 \pm 13.0$ & $-87.1 \pm 6.9$ \\
\hline
\end{tabular}


Table S8, contd.

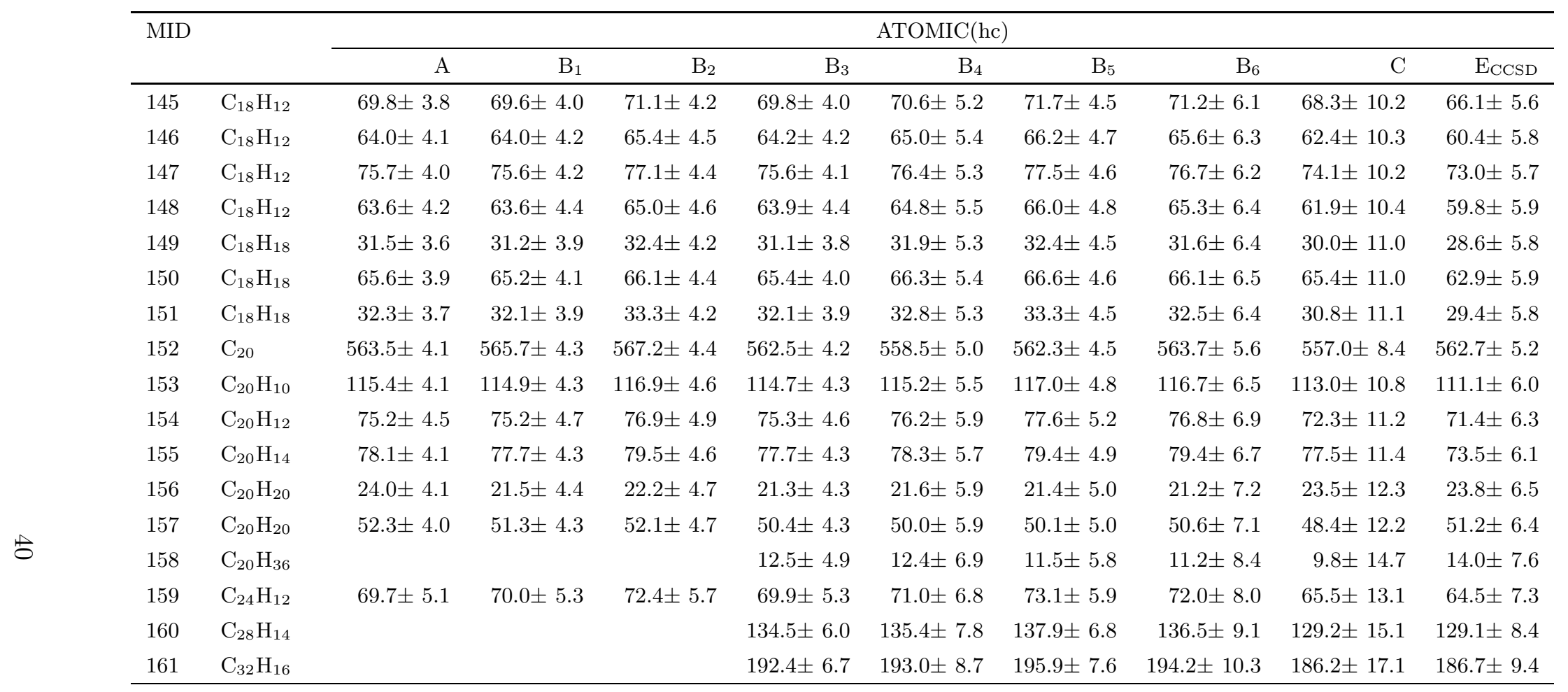

${ }^{a}$ Molecules are identified by their molecule index MID as specified in Table S1. Corrections to thermal enthalpy increments have been applied as described in the text. 
Table S9: ZPE-inclusive atomization energies (in $\mathrm{kcal} / \mathrm{mol}$ )

\begin{tabular}{|c|c|c|c|c|c|c|c|c|c|c|}
\hline \multirow[t]{2}{*}{ MID } & & \multicolumn{9}{|c|}{ ATOMIC(hc) } \\
\hline & & $\mathrm{A}$ & $\mathrm{B}_{1}$ & $\mathrm{~B}_{2}$ & $\mathrm{~B}_{3}$ & $\mathrm{~B}_{4}$ & $\mathrm{~B}_{5}$ & $\mathrm{~B}_{6}$ & $\mathrm{C}$ & $\mathrm{E}_{\mathrm{CCSD}}$ \\
\hline 1 & $\mathrm{CH}_{4}$ & $392.4 \pm 0.5$ & $392.3 \pm 0.5$ & $392.4 \pm 0.5$ & $392.3 \pm 0.5$ & $392.2 \pm 0.6$ & $392.2 \pm 0.6$ & $392.2 \pm 0.7$ & $391.9 \pm 1.2$ & $392.3 \pm 0.7$ \\
\hline 2 & $\mathrm{C}_{2} \mathrm{H}_{2}$ & $388.5 \pm 0.6$ & $388.4 \pm 0.6$ & $388.5 \pm 0.6$ & $388.4 \pm 0.6$ & $388.3 \pm 0.7$ & $388.3 \pm 0.6$ & $388.3 \pm 0.8$ & $388.0 \pm 1.2$ & $388.4 \pm 0.7$ \\
\hline 3 & $\mathrm{C}_{2} \mathrm{H}_{4}$ & $532.0 \pm 0.5$ & $531.9 \pm 0.5$ & $532.0 \pm 0.5$ & $531.9 \pm 0.5$ & $531.7 \pm 0.7$ & $531.8 \pm 0.6$ & $531.8 \pm 0.9$ & $531.4 \pm 1.5$ & $531.9 \pm 0.8$ \\
\hline 4 & $\mathrm{C}_{2} \mathrm{H}_{6}$ & $666.2 \pm 0.8$ & $666.1 \pm 0.8$ & $666.3 \pm 0.9$ & $666.0 \pm 0.8$ & $665.8 \pm 1.0$ & $666.0 \pm 0.9$ & $666.0 \pm 1.2$ & $665.5 \pm 2.0$ & $666.1 \pm 1.1$ \\
\hline 5 & $\mathrm{C}_{3} \mathrm{H}_{4}$ & $669.3 \pm 0.6$ & $669.5 \pm 0.6$ & $669.5 \pm 0.7$ & $669.4 \pm 0.6$ & $669.2 \pm 0.9$ & $669.4 \pm 0.7$ & $669.5 \pm 1.1$ & $669.0 \pm 1.9$ & $669.4 \pm 1.0$ \\
\hline 6 & $\mathrm{C}_{3} \mathrm{H}_{4}$ & $646.6 \pm 0.6$ & $646.4 \pm 0.6$ & $646.4 \pm 0.7$ & $646.3 \pm 0.6$ & $646.4 \pm 0.8$ & $646.5 \pm 0.7$ & $646.5 \pm 1.0$ & $646.4 \pm 1.8$ & $646.7 \pm 0.9$ \\
\hline 7 & $\mathrm{C}_{3} \mathrm{H}_{4}$ & $670.4 \pm 0.6$ & $670.5 \pm 0.7$ & $670.5 \pm 0.7$ & $670.5 \pm 0.7$ & $670.3 \pm 0.9$ & $670.4 \pm 0.8$ & $670.4 \pm 1.1$ & $669.8 \pm 1.9$ & $670.5 \pm 1.0$ \\
\hline 8 & $\mathrm{C}_{3} \mathrm{H}_{6}$ & $802.7 \pm 0.9$ & $802.4 \pm 0.9$ & $802.4 \pm 1.0$ & $802.2 \pm 0.9$ & $802.2 \pm 1.2$ & $802.4 \pm 1.0$ & $802.6 \pm 1.4$ & $802.5 \pm 2.3$ & $802.6 \pm 1.3$ \\
\hline 9 & $\mathrm{C}_{3} \mathrm{H}_{6}$ & $811.4 \pm 0.8$ & $811.4 \pm 0.8$ & $811.5 \pm 0.9$ & $811.3 \pm 0.8$ & $811.1 \pm 1.1$ & $811.2 \pm 0.9$ & $811.3 \pm 1.3$ & $810.6 \pm 2.3$ & $811.3 \pm 1.2$ \\
\hline 10 & $\mathrm{C}_{3} \mathrm{H}_{8}$ & $942.9 \pm 1.0$ & $942.8 \pm 1.1$ & $943.1 \pm 1.2$ & $942.8 \pm 1.1$ & $942.5 \pm 1.4$ & $942.7 \pm 1.2$ & $942.8 \pm 1.7$ & $941.9 \pm 2.7$ & $942.8 \pm 1.5$ \\
\hline 11 & $\mathrm{C}_{4} \mathrm{H}_{2}$ & $673.2 \pm 1.1$ & $673.5 \pm 1.1$ & $673.5 \pm 1.1$ & $673.6 \pm 1.1$ & $673.4 \pm 1.3$ & $673.3 \pm 1.2$ & $672.9 \pm 1.4$ & $673.7 \pm 2.1$ & $673.4 \pm 1.3$ \\
\hline 12 & $\mathrm{C}_{4} \mathrm{H}_{4}$ & $747.5 \pm 0.9$ & $747.0 \pm 0.9$ & $747.2 \pm 1.0$ & $747.5 \pm 0.9$ & $748.2 \pm 1.1$ & $748.0 \pm 1.0$ & $748.2 \pm 1.3$ & $748.4 \pm 2.2$ & $747.0 \pm 1.2$ \\
\hline 13 & $\mathrm{C}_{4} \mathrm{H}_{4}$ & $807.6 \pm 0.8$ & $808.0 \pm 0.9$ & $807.9 \pm 0.9$ & $807.9 \pm 0.9$ & $807.8 \pm 1.1$ & $807.8 \pm 1.0$ & $807.7 \pm 1.4$ & $807.9 \pm 2.3$ & $807.4 \pm 1.2$ \\
\hline 14 & $\mathrm{C}_{4} \mathrm{H}_{4}$ & $781.8 \pm 0.8$ & $781.4 \pm 0.9$ & $781.7 \pm 0.9$ & $781.5 \pm 0.9$ & $781.8 \pm 1.1$ & $781.8 \pm 1.0$ & $781.6 \pm 1.3$ & $781.5 \pm 2.2$ & $780.5 \pm 1.2$ \\
\hline 15 & $\mathrm{C}_{4} \mathrm{H}_{4}$ & $815.4 \pm 0.9$ & $815.6 \pm 1.0$ & $815.6 \pm 1.0$ & $815.6 \pm 1.0$ & $815.4 \pm 1.2$ & $815.4 \pm 1.1$ & $815.3 \pm 1.4$ & $815.1 \pm 2.3$ & $815.4 \pm 1.3$ \\
\hline 16 & $\mathrm{C}_{4} \mathrm{H}_{4}$ & $791.9 \pm 0.7$ & $791.9 \pm 0.8$ & $791.8 \pm 0.8$ & $791.8 \pm 0.8$ & $791.8 \pm 1.1$ & $791.7 \pm 0.9$ & $792.0 \pm 1.3$ & $791.4 \pm 2.2$ & $792.1 \pm 1.2$ \\
\hline 17 & $\mathrm{C}_{4} \mathrm{H}_{4}$ & $755.7 \pm 0.8$ & $755.1 \pm 0.8$ & $754.8 \pm 0.9$ & $755.1 \pm 0.8$ & $755.7 \pm 1.1$ & $755.9 \pm 0.9$ & $756.3 \pm 1.3$ & $757.4 \pm 2.1$ & $755.8 \pm 1.1$ \\
\hline 18 & $\mathrm{C}_{4} \mathrm{H}_{6}$ & $947.5 \pm 0.9$ & $947.8 \pm 0.9$ & $947.8 \pm 1.0$ & $947.7 \pm 0.9$ & $947.5 \pm 1.3$ & $947.6 \pm 1.1$ & $947.8 \pm 1.5$ & $947.2 \pm 2.7$ & $947.7 \pm 1.4$ \\
\hline 19 & $\mathrm{C}_{4} \mathrm{H}_{6}$ & $959.6 \pm 0.9$ & $959.5 \pm 1.0$ & $959.6 \pm 1.0$ & $959.5 \pm 0.9$ & $959.2 \pm 1.3$ & $959.3 \pm 1.1$ & $959.4 \pm 1.6$ & $958.8 \pm 2.7$ & $959.4 \pm 1.4$ \\
\hline 20 & $\mathrm{C}_{4} \mathrm{H}_{6}$ & $951.4 \pm 0.8$ & $951.7 \pm 0.9$ & $951.6 \pm 0.9$ & $951.8 \pm 0.9$ & $951.5 \pm 1.2$ & $951.6 \pm 1.0$ & $951.6 \pm 1.5$ & $950.7 \pm 2.6$ & $951.7 \pm 1.4$ \\
\hline 21 & $\mathrm{C}_{4} \mathrm{H}_{6}$ & $931.9 \pm 0.9$ & $931.5 \pm 0.9$ & $931.4 \pm 1.0$ & $931.5 \pm 0.9$ & $931.7 \pm 1.3$ & $931.8 \pm 1.1$ & $932.0 \pm 1.5$ & $932.2 \pm 2.6$ & $932.0 \pm 1.4$ \\
\hline 22 & $\mathrm{C}_{4} \mathrm{H}_{6}$ & $947.3 \pm 0.8$ & $947.2 \pm 0.9$ & $947.3 \pm 1.0$ & $947.1 \pm 0.9$ & $947.1 \pm 1.2$ & $947.2 \pm 1.0$ & $947.1 \pm 1.5$ & $946.4 \pm 2.6$ & $947.3 \pm 1.4$ \\
\hline 23 & $\mathrm{C}_{4} \mathrm{H}_{6}$ & $939.7 \pm 0.9$ & $939.6 \pm 0.9$ & $939.5 \pm 1.0$ & $939.6 \pm 0.9$ & $939.5 \pm 1.3$ & $939.7 \pm 1.1$ & $939.8 \pm 1.5$ & $939.4 \pm 2.6$ & $939.6 \pm 1.4$ \\
\hline 24 & $\mathrm{C}_{4} \mathrm{H}_{8}$ & $1080.7 \pm 1.2$ & $1080.6 \pm 1.2$ & $1080.8 \pm 1.3$ & $1080.5 \pm 1.2$ & $1080.4 \pm 1.6$ & $1080.6 \pm 1.4$ & $1080.5 \pm 1.9$ & $1080.1 \pm 3.1$ & $1080.5 \pm 1.7$ \\
\hline 25 & $\mathrm{C}_{4} \mathrm{H}_{8}$ & $1092.0 \pm 1.0$ & $1091.9 \pm 1.1$ & $1092.0 \pm 1.2$ & $1091.9 \pm 1.1$ & $1091.6 \pm 1.5$ & $1091.8 \pm 1.2$ & $1092.0 \pm 1.8$ & $1091.0 \pm 3.1$ & $1091.9 \pm 1.6$ \\
\hline 26 & $\mathrm{C}_{4} \mathrm{H}_{10}$ & $1221.6 \pm 1.3$ & $1221.6 \pm 1.3$ & $1221.8 \pm 1.4$ & $1221.6 \pm 1.3$ & $1221.2 \pm 1.8$ & $1221.6 \pm 1.5$ & $1221.6 \pm 2.1$ & $1220.4 \pm 3.5$ & $1221.5 \pm 1.9$ \\
\hline 27 & $\mathrm{C}_{4} \mathrm{H}_{10}$ & $1219.8 \pm 1.3$ & $1219.8 \pm 1.4$ & $1220.0 \pm 1.5$ & $1219.7 \pm 1.4$ & $1219.4 \pm 1.8$ & $1219.7 \pm 1.5$ & $1219.8 \pm 2.1$ & $1218.6 \pm 3.5$ & $1219.7 \pm 1.9$ \\
\hline 28 & $\mathrm{C}_{5} \mathrm{H}_{4}$ & $946.3 \pm 1.0$ & $946.6 \pm 1.1$ & $946.5 \pm 1.1$ & $946.8 \pm 1.1$ & $946.5 \pm 1.4$ & $946.5 \pm 1.2$ & $946.6 \pm 1.6$ & $946.0 \pm 2.7$ & $946.7 \pm 1.5$ \\
\hline
\end{tabular}


Table S9, contd.

\begin{tabular}{|c|c|c|c|c|c|c|c|c|c|c|}
\hline \multirow[t]{2}{*}{ MID } & & \multicolumn{9}{|c|}{ ATOMIC(hc) } \\
\hline & & $\mathrm{A}$ & $\mathrm{B}_{1}$ & $\mathrm{~B}_{2}$ & $\mathrm{~B}_{3}$ & $\mathrm{~B}_{4}$ & $\mathrm{~B}_{5}$ & $\mathrm{~B}_{6}$ & $\mathrm{C}$ & $\mathrm{E}_{\mathrm{CCSD}}$ \\
\hline 29 & $\mathrm{C}_{5} \mathrm{H}_{4}$ & $947.6 \pm 1.0$ & $948.2 \pm 1.1$ & $948.1 \pm 1.1$ & $948.0 \pm 1.0$ & $948.0 \pm 1.4$ & $947.8 \pm 1.2$ & $947.6 \pm 1.6$ & $948.8 \pm 2.7$ & $947.5 \pm 1.5$ \\
\hline 30 & $\mathrm{C}_{5} \mathrm{H}_{4}$ & $900.9 \pm 0.8$ & $900.8 \pm 0.9$ & $900.7 \pm 1.0$ & $900.7 \pm 0.9$ & $901.0 \pm 1.2$ & $900.9 \pm 1.0$ & $900.9 \pm 1.5$ & $900.9 \pm 2.5$ & $900.8 \pm 1.3$ \\
\hline 31 & $\mathrm{C}_{5} \mathrm{H}_{6}$ & $1068.5 \pm 1.0$ & $1067.7 \pm 1.0$ & $1068.0 \pm 1.1$ & $1068.2 \pm 1.0$ & $1068.9 \pm 1.4$ & $1068.7 \pm 1.2$ & $1069.0 \pm 1.7$ & $1069.9 \pm 3.0$ & $1068.5 \pm 1.6$ \\
\hline 32 & $\mathrm{C}_{5} \mathrm{H}_{6}$ & $1123.9 \pm 1.1$ & $1123.8 \pm 1.1$ & $1123.7 \pm 1.2$ & $1123.7 \pm 1.1$ & $1123.5 \pm 1.5$ & $1123.6 \pm 1.3$ & $1123.7 \pm 1.8$ & $1122.8 \pm 3.2$ & $1124.0 \pm 1.7$ \\
\hline 33 & $\mathrm{C}_{5} \mathrm{H}_{6}$ & $1096.1 \pm 1.0$ & $1096.1 \pm 1.1$ & $1096.2 \pm 1.2$ & $1096.1 \pm 1.1$ & $1096.0 \pm 1.5$ & $1096.0 \pm 1.2$ & $1096.0 \pm 1.8$ & $1095.5 \pm 3.1$ & $1096.0 \pm 1.6$ \\
\hline 34 & $\mathrm{C}_{5} \mathrm{H}_{6}$ & $1077.0 \pm 0.9$ & $1076.6 \pm 1.0$ & $1076.6 \pm 1.1$ & $1076.6 \pm 1.0$ & $1076.9 \pm 1.4$ & $1077.0 \pm 1.2$ & $1076.9 \pm 1.7$ & $1077.1 \pm 3.0$ & $1076.9 \pm 1.5$ \\
\hline 35 & $\mathrm{C}_{5} \mathrm{H}_{6}$ & $1085.2 \pm 1.0$ & $1085.2 \pm 1.0$ & $1085.0 \pm 1.1$ & $1085.1 \pm 1.0$ & $1085.0 \pm 1.4$ & $1085.1 \pm 1.2$ & $1085.3 \pm 1.7$ & $1085.1 \pm 3.0$ & $1085.3 \pm 1.6$ \\
\hline 36 & $\mathrm{C}_{5} \mathrm{H}_{8}$ & $1232.7 \pm 1.0$ & $1232.7 \pm 1.1$ & $1232.9 \pm 1.2$ & $1232.7 \pm 1.1$ & $1232.4 \pm 1.6$ & $1232.5 \pm 1.3$ & $1232.7 \pm 2.0$ & $1231.7 \pm 3.4$ & $1232.7 \pm 1.8$ \\
\hline 37 & $\mathrm{C}_{5} \mathrm{H}_{8}$ & $1239.7 \pm 1.1$ & $1239.6 \pm 1.2$ & $1239.7 \pm 1.3$ & $1239.6 \pm 1.2$ & $1239.3 \pm 1.6$ & $1239.5 \pm 1.4$ & $1239.6 \pm 2.0$ & $1238.9 \pm 3.5$ & $1239.5 \pm 1.8$ \\
\hline 38 & $\mathrm{C}_{5} \mathrm{H}_{8}$ & $1213.1 \pm 1.2$ & $1212.9 \pm 1.3$ & $1212.6 \pm 1.4$ & $1212.7 \pm 1.2$ & $1212.8 \pm 1.7$ & $1213.0 \pm 1.4$ & $1213.2 \pm 2.0$ & $1212.8 \pm 3.4$ & $1212.9 \pm 1.8$ \\
\hline 39 & $\mathrm{C}_{5} \mathrm{H}_{10}$ & $1377.0 \pm 1.4$ & $1377.1 \pm 1.4$ & $1377.3 \pm 1.5$ & $1376.9 \pm 1.4$ & $1376.6 \pm 1.9$ & $1377.0 \pm 1.6$ & $1377.0 \pm 2.3$ & $1375.9 \pm 3.9$ & $1377.0 \pm 2.1$ \\
\hline 40 & $\mathrm{C}_{5} \mathrm{H}_{12}$ & $1501.3 \pm 1.5$ & $1501.4 \pm 1.6$ & $1501.6 \pm 1.7$ & $1501.5 \pm 1.5$ & $1501.2 \pm 2.1$ & $1501.6 \pm 1.8$ & $1501.6 \pm 2.5$ & $1500.2 \pm 4.3$ & $1501.3 \pm 2.3$ \\
\hline 41 & $\mathrm{C}_{5} \mathrm{H}_{12}$ & $1496.7 \pm 1.6$ & $1496.7 \pm 1.7$ & $1497.0 \pm 1.8$ & $1496.7 \pm 1.6$ & $1496.3 \pm 2.2$ & $1496.6 \pm 1.9$ & $1496.7 \pm 2.6$ & $1495.3 \pm 4.3$ & $1496.5 \pm 2.3$ \\
\hline 42 & $\mathrm{C}_{6} \mathrm{H}_{2}$ & $959.3 \pm 1.5$ & $959.7 \pm 1.5$ & $959.7 \pm 1.6$ & $960.0 \pm 1.5$ & $959.7 \pm 1.8$ & $959.3 \pm 1.6$ & $958.5 \pm 2.0$ & $961.3 \pm 2.9$ & $959.8 \pm 1.8$ \\
\hline 43 & $\mathrm{C}_{6} \mathrm{H}_{4}$ & $1055.3 \pm 1.1$ & $1055.0 \pm 1.2$ & $1055.1 \pm 1.3$ & $1055.3 \pm 1.2$ & $1056.1 \pm 1.5$ & $1055.6 \pm 1.3$ & $1055.1 \pm 1.8$ & $1056.6 \pm 3.0$ & $1055.6 \pm 1.6$ \\
\hline 44 & $\mathrm{C}_{6} \mathrm{H}_{4}$ & $1099.1 \pm 1.5$ & $1099.5 \pm 1.5$ & $1099.4 \pm 1.6$ & $1099.6 \pm 1.5$ & $1099.3 \pm 1.8$ & $1099.2 \pm 1.6$ & $1099.1 \pm 2.1$ & $1099.3 \pm 3.2$ & $1099.4 \pm 1.9$ \\
\hline 45 & $\mathrm{C}_{6} \mathrm{H}_{4}$ & $1087.8 \pm 1.2$ & $1088.5 \pm 1.3$ & $1088.4 \pm 1.3$ & $1088.3 \pm 1.3$ & $1088.4 \pm 1.6$ & $1088.0 \pm 1.4$ & $1087.7 \pm 1.9$ & $1089.9 \pm 3.1$ & $1087.3 \pm 1.7$ \\
\hline 46 & $\mathrm{C}_{6} \mathrm{H}_{4}$ & $1114.2 \pm 1.1$ & $1113.8 \pm 1.1$ & $1113.5 \pm 1.2$ & $1114.2 \pm 1.1$ & $1114.5 \pm 1.5$ & $1114.1 \pm 1.3$ & $1113.7 \pm 1.8$ & $1114.5 \pm 3.1$ & $1113.6 \pm 1.7$ \\
\hline 47 & $\mathrm{C}_{6} \mathrm{H}_{4}$ & $1099.4 \pm 1.4$ & $1099.8 \pm 1.5$ & $1099.7 \pm 1.5$ & $1099.9 \pm 1.5$ & $1099.6 \pm 1.8$ & $1099.5 \pm 1.6$ & $1099.4 \pm 2.0$ & $1099.5 \pm 3.2$ & $1099.6 \pm 1.9$ \\
\hline 48 & $\mathrm{C}_{6} \mathrm{H}_{4}$ & $1040.6 \pm 1.1$ & $1040.7 \pm 1.2$ & $1040.3 \pm 1.2$ & $1040.5 \pm 1.2$ & $1040.9 \pm 1.5$ & $1040.6 \pm 1.3$ & $1040.8 \pm 1.8$ & $1040.8 \pm 2.9$ & $1040.8 \pm 1.6$ \\
\hline 49 & $\mathrm{C}_{6} \mathrm{H}_{6}$ & $1238.4 \pm 1.2$ & $1238.8 \pm 1.3$ & $1238.7 \pm 1.4$ & $1239.0 \pm 1.2$ & $1238.6 \pm 1.7$ & $1238.4 \pm 1.4$ & $1238.1 \pm 2.0$ & $1239.2 \pm 3.5$ & $1238.9 \pm 1.8$ \\
\hline 50 & $\mathrm{C}_{6} \mathrm{H}_{6}$ & $1186.4 \pm 1.1$ & $1186.3 \pm 1.1$ & $1186.2 \pm 1.2$ & $1186.3 \pm 1.1$ & $1186.6 \pm 1.6$ & $1186.6 \pm 1.3$ & $1186.7 \pm 1.9$ & $1186.7 \pm 3.3$ & $1186.9 \pm 1.7$ \\
\hline 51 & $\mathrm{C}_{6} \mathrm{H}_{6}$ & $1244.9 \pm 1.3$ & $1244.9 \pm 1.3$ & $1244.9 \pm 1.4$ & $1245.0 \pm 1.3$ & $1244.9 \pm 1.8$ & $1244.7 \pm 1.5$ & $1244.7 \pm 2.1$ & $1244.5 \pm 3.5$ & $1244.6 \pm 1.9$ \\
\hline 52 & $\mathrm{C}_{6} \mathrm{H}_{6}$ & $1220.6 \pm 1.2$ & $1220.6 \pm 1.3$ & $1220.4 \pm 1.4$ & $1220.7 \pm 1.2$ & $1220.7 \pm 1.7$ & $1220.5 \pm 1.4$ & $1220.2 \pm 2.0$ & $1220.8 \pm 3.4$ & $1219.8 \pm 1.8$ \\
\hline 53 & $\mathrm{C}_{6} \mathrm{H}_{6}$ & $1306.0 \pm 1.3$ & $1306.0 \pm 1.4$ & $1305.7 \pm 1.5$ & $1305.8 \pm 1.4$ & $1305.4 \pm 1.8$ & $1305.3 \pm 1.5$ & $1305.3 \pm 2.2$ & $1304.9 \pm 3.7$ & $1307.2 \pm 2.0$ \\
\hline 54 & $\mathrm{C}_{6} \mathrm{H}_{6}$ & $1233.0 \pm 1.1$ & $1232.7 \pm 1.2$ & $1232.5 \pm 1.3$ & $1232.7 \pm 1.2$ & $1233.1 \pm 1.6$ & $1233.1 \pm 1.4$ & $1233.2 \pm 2.0$ & $1233.3 \pm 3.4$ & $1233.3 \pm 1.8$ \\
\hline 55 & $\mathrm{C}_{6} \mathrm{H}_{6}$ & $1229.3 \pm 1.1$ & $1229.2 \pm 1.2$ & $1229.3 \pm 1.3$ & $1229.2 \pm 1.2$ & $1229.4 \pm 1.6$ & $1229.4 \pm 1.4$ & $1229.1 \pm 2.0$ & $1229.0 \pm 3.4$ & $1229.4 \pm 1.8$ \\
\hline 56 & $\mathrm{C}_{6} \mathrm{H}_{6}$ & $1274.5 \pm 1.3$ & $1274.5 \pm 1.4$ & $1274.3 \pm 1.5$ & $1274.5 \pm 1.4$ & $1274.2 \pm 1.8$ & $1274.1 \pm 1.6$ & $1274.2 \pm 2.1$ & $1273.7 \pm 3.6$ & $1274.6 \pm 2.0$ \\
\hline 57 & $\mathrm{C}_{6} \mathrm{H}_{6}$ & $1192.2 \pm 1.1$ & $1191.8 \pm 1.2$ & $1191.7 \pm 1.3$ & $1191.7 \pm 1.2$ & $1192.4 \pm 1.6$ & $1192.5 \pm 1.3$ & $1192.4 \pm 1.9$ & $1193.2 \pm 3.3$ & $1191.9 \pm 1.7$ \\
\hline
\end{tabular}


Table S9, contd.

\begin{tabular}{|c|c|c|c|c|c|c|c|c|c|c|}
\hline \multirow[t]{2}{*}{ MID } & & \multicolumn{9}{|c|}{ ATOMIC(hc) } \\
\hline & & $\mathrm{A}$ & $\mathrm{B}_{1}$ & $\mathrm{~B}_{2}$ & $\mathrm{~B}_{3}$ & $\mathrm{~B}_{4}$ & $\mathrm{~B}_{5}$ & $\mathrm{~B}_{6}$ & $\mathrm{C}$ & $\mathrm{E}_{\mathrm{CCSD}}$ \\
\hline 58 & $\mathrm{C}_{6} \mathrm{H}_{8}$ & $1401.7 \pm 1.3$ & $1401.7 \pm 1.4$ & $1401.6 \pm 1.5$ & $1401.7 \pm 1.3$ & $1401.4 \pm 1.9$ & $1401.5 \pm 1.6$ & $1401.7 \pm 2.3$ & $1400.6 \pm 3.9$ & $1401.6 \pm 2.0$ \\
\hline 59 & $\mathrm{C}_{6} \mathrm{H}_{8}$ & $1401.7 \pm 1.2$ & $1401.9 \pm 1.3$ & $1401.8 \pm 1.4$ & $1401.8 \pm 1.3$ & $1401.5 \pm 1.8$ & $1401.7 \pm 1.5$ & $1402.0 \pm 2.2$ & $1400.8 \pm 3.9$ & $1401.8 \pm 2.0$ \\
\hline 60 & $\mathrm{C}_{6} \mathrm{H}_{8}$ & $1388.2 \pm 1.4$ & $1388.2 \pm 1.4$ & $1388.3 \pm 1.5$ & $1388.2 \pm 1.4$ & $1387.8 \pm 1.9$ & $1387.9 \pm 1.6$ & $1388.1 \pm 2.3$ & $1387.4 \pm 3.9$ & $1388.0 \pm 2.1$ \\
\hline 61 & $\mathrm{C}_{6} \mathrm{H}_{10}$ & $1529.8 \pm 1.4$ & $1529.9 \pm 1.5$ & $1530.0 \pm 1.6$ & $1529.9 \pm 1.5$ & $1529.5 \pm 2.1$ & $1529.8 \pm 1.7$ & $1530.0 \pm 2.5$ & $1528.7 \pm 4.3$ & $1529.8 \pm 2.2$ \\
\hline 62 & $\mathrm{C}_{6} \mathrm{H}_{12}$ & $1659.5 \pm 1.6$ & $1659.7 \pm 1.7$ & $1659.9 \pm 1.8$ & $1659.5 \pm 1.7$ & $1659.2 \pm 2.3$ & $1659.6 \pm 2.0$ & $1659.6 \pm 2.8$ & $1658.1 \pm 4.7$ & $1659.5 \pm 2.5$ \\
\hline 63 & $\mathrm{C}_{6} \mathrm{H}_{14}$ & $1774.2 \pm 1.8$ & $1774.3 \pm 1.9$ & $1774.6 \pm 2.0$ & $1774.2 \pm 1.9$ & $1773.7 \pm 2.5$ & $1774.2 \pm 2.1$ & $1774.4 \pm 3.0$ & $1772.9 \pm 5.1$ & $1774.0 \pm 2.7$ \\
\hline 64 & $\mathrm{C}_{6} \mathrm{H}_{14}$ & $1773.7 \pm 1.8$ & $1773.7 \pm 1.9$ & $1774.0 \pm 2.1$ & $1773.7 \pm 1.9$ & $1773.2 \pm 2.5$ & $1773.6 \pm 2.2$ & $1773.8 \pm 3.0$ & $1772.0 \pm 5.1$ & $1773.4 \pm 2.7$ \\
\hline 65 & $\mathrm{C}_{7} \mathrm{H}_{4}$ & $1210.2 \pm 1.4$ & $1210.5 \pm 1.4$ & $1210.3 \pm 1.5$ & $1210.7 \pm 1.4$ & $1210.7 \pm 1.8$ & $1210.4 \pm 1.6$ & $1210.5 \pm 2.1$ & $1210.8 \pm 3.4$ & $1210.8 \pm 1.9$ \\
\hline 66 & $\mathrm{C}_{7} \mathrm{H}_{4}$ & $1228.7 \pm 1.4$ & $1229.6 \pm 1.5$ & $1229.5 \pm 1.6$ & $1229.4 \pm 1.5$ & $1229.5 \pm 1.9$ & $1229.0 \pm 1.6$ & $1228.6 \pm 2.2$ & $1231.5 \pm 3.5$ & $1228.3 \pm 2.0$ \\
\hline 67 & $\mathrm{C}_{7} \mathrm{H}_{6}$ & $1365.5 \pm 1.4$ & $1365.6 \pm 1.5$ & $1365.3 \pm 1.6$ & $1365.8 \pm 1.5$ & $1365.6 \pm 1.9$ & $1365.5 \pm 1.7$ & $1365.7 \pm 2.3$ & $1365.8 \pm 3.9$ & $1365.8 \pm 2.1$ \\
\hline 68 & $\mathrm{C}_{7} \mathrm{H}_{6}$ & $1405.0 \pm 1.3$ & $1405.0 \pm 1.4$ & $1404.5 \pm 1.5$ & $1405.0 \pm 1.4$ & $1405.0 \pm 1.9$ & $1404.8 \pm 1.6$ & $1404.6 \pm 2.3$ & $1405.2 \pm 3.9$ & $1406.2 \pm 2.1$ \\
\hline 69 & $\mathrm{C}_{7} \mathrm{H}_{6}$ & $1411.6 \pm 1.4$ & $1411.9 \pm 1.5$ & $1411.6 \pm 1.6$ & $1411.8 \pm 1.5$ & $1411.6 \pm 2.0$ & $1411.4 \pm 1.7$ & $1411.6 \pm 2.4$ & $1411.5 \pm 4.0$ & $1412.1 \pm 2.2$ \\
\hline 70 & $\mathrm{C}_{7} \mathrm{H}_{6}$ & $1389.8 \pm 1.4$ & $1389.7 \pm 1.5$ & $1389.4 \pm 1.6$ & $1389.6 \pm 1.5$ & $1389.7 \pm 1.9$ & $1389.6 \pm 1.7$ & $1389.6 \pm 2.3$ & $1389.4 \pm 3.9$ & $1390.3 \pm 2.1$ \\
\hline 71 & $\mathrm{C}_{7} \mathrm{H}_{8}$ & $1553.0 \pm 1.6$ & $1552.9 \pm 1.6$ & $1552.8 \pm 1.8$ & $1552.9 \pm 1.6$ & $1552.6 \pm 2.2$ & $1552.7 \pm 1.9$ & $1552.8 \pm 2.6$ & $1552.4 \pm 4.4$ & $1553.3 \pm 2.4$ \\
\hline 72 & $\mathrm{C}_{7} \mathrm{H}_{8}$ & $1539.5 \pm 1.4$ & $1539.4 \pm 1.5$ & $1539.4 \pm 1.6$ & $1539.4 \pm 1.5$ & $1539.5 \pm 2.0$ & $1539.6 \pm 1.7$ & $1539.4 \pm 2.5$ & $1539.0 \pm 4.3$ & $1539.8 \pm 2.2$ \\
\hline 73 & $\mathrm{C}_{7} \mathrm{H}_{8}$ & $1547.1 \pm 1.5$ & $1546.9 \pm 1.6$ & $1546.6 \pm 1.7$ & $1546.8 \pm 1.6$ & $1546.7 \pm 2.1$ & $1546.8 \pm 1.8$ & $1546.9 \pm 2.5$ & $1546.8 \pm 4.4$ & $1547.2 \pm 2.3$ \\
\hline 74 & $\mathrm{C}_{7} \mathrm{H}_{8}$ & $1516.7 \pm 1.3$ & $1516.4 \pm 1.4$ & $1516.1 \pm 1.5$ & $1516.4 \pm 1.4$ & $1516.8 \pm 2.0$ & $1517.0 \pm 1.7$ & $1517.0 \pm 2.4$ & $1517.1 \pm 4.2$ & $1516.8 \pm 2.2$ \\
\hline 75 & $\mathrm{C}_{7} \mathrm{H}_{8}$ & $1543.2 \pm 1.4$ & $1543.0 \pm 1.5$ & $1542.7 \pm 1.6$ & $1542.9 \pm 1.5$ & $1542.9 \pm 2.1$ & $1542.9 \pm 1.7$ & $1543.0 \pm 2.5$ & $1542.5 \pm 4.3$ & $1543.5 \pm 2.3$ \\
\hline 76 & $\mathrm{C}_{7} \mathrm{H}_{8}$ & $1585.7 \pm 1.5$ & $1585.8 \pm 1.6$ & $1585.4 \pm 1.7$ & $1585.6 \pm 1.5$ & $1585.2 \pm 2.1$ & $1585.1 \pm 1.8$ & $1585.2 \pm 2.6$ & $1584.8 \pm 4.4$ & $1587.0 \pm 2.3$ \\
\hline 77 & $\mathrm{C}_{7} \mathrm{H}_{12}$ & $1813.3 \pm 1.6$ & $1813.5 \pm 1.8$ & $1813.6 \pm 1.9$ & $1813.4 \pm 1.7$ & $1813.1 \pm 2.4$ & $1813.5 \pm 2.0$ & $1813.5 \pm 2.9$ & $1812.4 \pm 5.1$ & $1813.5 \pm 2.7$ \\
\hline 78 & $\mathrm{C}_{7} \mathrm{H}_{16}$ & $2050.6 \pm 2.1$ & $2050.7 \pm 2.2$ & $2051.1 \pm 2.4$ & $2050.6 \pm 2.2$ & $2050.1 \pm 2.9$ & $2050.6 \pm 2.5$ & $2050.8 \pm 3.5$ & $2048.8 \pm 5.9$ & $2050.4 \pm 3.2$ \\
\hline 79 & $\mathrm{C}_{8} \mathrm{H}_{2}$ & $1245.9 \pm 2.0$ & $1246.5 \pm 2.1$ & $1246.4 \pm 2.1$ & $1246.8 \pm 2.1$ & $1246.5 \pm 2.3$ & $1245.9 \pm 2.2$ & $1244.7 \pm 2.6$ & $1249.1 \pm 3.8$ & $1246.6 \pm 2.5$ \\
\hline 80 & $\mathrm{C}_{8} \mathrm{H}_{4}$ & $1365.0 \pm 1.7$ & $1365.3 \pm 1.7$ & $1364.9 \pm 1.8$ & $1365.6 \pm 1.7$ & $1366.1 \pm 2.1$ & $1365.4 \pm 1.9$ & $1365.4 \pm 2.4$ & $1366.7 \pm 3.9$ & $1363.0 \pm 2.3$ \\
\hline 81 & $\mathrm{C}_{8} \mathrm{H}_{4}$ & $1389.7 \pm 1.7$ & $1389.7 \pm 1.7$ & $1389.4 \pm 1.8$ & $1390.3 \pm 1.7$ & $1390.4 \pm 2.1$ & $1389.8 \pm 1.9$ & $1389.4 \pm 2.5$ & $1390.6 \pm 4.0$ & $1389.4 \pm 2.3$ \\
\hline 82 & $\mathrm{C}_{8} \mathrm{H}_{4}$ & $1369.7 \pm 1.6$ & $1370.7 \pm 1.7$ & $1370.5 \pm 1.8$ & $1370.5 \pm 1.7$ & $1370.6 \pm 2.1$ & $1369.9 \pm 1.9$ & $1369.5 \pm 2.4$ & $1373.3 \pm 3.9$ & $1369.1 \pm 2.3$ \\
\hline 83 & $\mathrm{C}_{8} \mathrm{H}_{6}$ & $1568.2 \pm 1.7$ & $1568.2 \pm 1.8$ & $1567.9 \pm 1.9$ & $1568.1 \pm 1.7$ & $1568.1 \pm 2.3$ & $1567.7 \pm 2.0$ & $1567.5 \pm 2.7$ & $1568.1 \pm 4.4$ & $1568.6 \pm 2.4$ \\
\hline 84 & $\mathrm{C}_{8} \mathrm{H}_{6}$ & $1506.4 \pm 1.4$ & $1506.4 \pm 1.5$ & $1505.8 \pm 1.6$ & $1506.6 \pm 1.4$ & $1507.1 \pm 2.0$ & $1506.6 \pm 1.7$ & $1506.3 \pm 2.4$ & $1507.9 \pm 4.2$ & $1507.7 \pm 2.2$ \\
\hline 85 & $\mathrm{C}_{8} \mathrm{H}_{6}$ & $1576.6 \pm 1.7$ & $1576.6 \pm 1.8$ & $1576.2 \pm 1.9$ & $1576.6 \pm 1.8$ & $1576.6 \pm 2.3$ & $1576.2 \pm 2.0$ & $1576.2 \pm 2.7$ & $1576.2 \pm 4.5$ & $1576.5 \pm 2.5$ \\
\hline 86 & $\mathrm{C}_{8} \mathrm{H}_{8}$ & $1696.2 \pm 1.8$ & $1696.3 \pm 1.9$ & $1696.0 \pm 2.0$ & $1696.3 \pm 1.9$ & $1696.0 \pm 2.4$ & $1695.9 \pm 2.1$ & $1696.3 \pm 2.9$ & $1696.0 \pm 4.8$ & $1696.3 \pm 2.6$ \\
\hline
\end{tabular}


Table S9, contd.

\begin{tabular}{|c|c|c|c|c|c|c|c|c|c|c|}
\hline \multirow[t]{2}{*}{ MID } & & \multicolumn{9}{|c|}{ ATOMIC(hc) } \\
\hline & & $\mathrm{A}$ & $\mathrm{B}_{1}$ & $\mathrm{~B}_{2}$ & $\mathrm{~B}_{3}$ & $\mathrm{~B}_{4}$ & $\mathrm{~B}_{5}$ & $\mathrm{~B}_{6}$ & $\mathrm{C}$ & $\mathrm{E}_{\mathrm{CCSD}}$ \\
\hline 87 & $\mathrm{C}_{8} \mathrm{H}_{8}$ & $1719.8 \pm 1.6$ & $1719.8 \pm 1.7$ & $1719.5 \pm 1.8$ & $1719.7 \pm 1.7$ & $1719.5 \pm 2.3$ & $1719.3 \pm 2.0$ & $1719.3 \pm 2.8$ & $1719.3 \pm 4.8$ & $1721.0 \pm 2.5$ \\
\hline 88 & $\mathrm{C}_{8} \mathrm{H}_{8}$ & $1621.4 \pm 1.6$ & $1620.9 \pm 1.7$ & $1621.2 \pm 1.8$ & $1620.8 \pm 1.7$ & $1621.5 \pm 2.3$ & $1621.6 \pm 1.9$ & $1621.0 \pm 2.7$ & $1622.2 \pm 4.6$ & $1620.1 \pm 2.4$ \\
\hline 89 & $\mathrm{C}_{8} \mathrm{H}_{8}$ & $1714.6 \pm 1.9$ & $1714.6 \pm 1.9$ & $1714.3 \pm 2.1$ & $1714.7 \pm 1.9$ & $1714.4 \pm 2.5$ & $1714.2 \pm 2.2$ & $1714.5 \pm 2.9$ & $1714.4 \pm 4.9$ & $1714.0 \pm 2.7$ \\
\hline 90 & $\mathrm{C}_{8} \mathrm{H}_{8}$ & $1732.3 \pm 1.7$ & $1732.4 \pm 1.8$ & $1732.0 \pm 1.9$ & $1732.2 \pm 1.8$ & $1731.7 \pm 2.4$ & $1731.6 \pm 2.0$ & $1731.7 \pm 2.9$ & $1731.6 \pm 4.9$ & $1733.5 \pm 2.6$ \\
\hline 91 & $\mathrm{C}_{8} \mathrm{H}_{10}$ & $1817.2 \pm 1.8$ & $1817.3 \pm 1.9$ & $1817.3 \pm 2.1$ & $1817.3 \pm 1.9$ & $1816.8 \pm 2.5$ & $1816.8 \pm 2.2$ & $1817.1 \pm 3.0$ & $1816.3 \pm 5.1$ & $1816.9 \pm 2.8$ \\
\hline 92 & $\mathrm{C}_{8} \mathrm{H}_{14}$ & $2094.5 \pm 1.9$ & $2094.7 \pm 2.1$ & $2094.9 \pm 2.2$ & $2094.6 \pm 2.0$ & $2094.2 \pm 2.8$ & $2094.8 \pm 2.4$ & $2094.7 \pm 3.4$ & $2093.4 \pm 5.9$ & $2094.5 \pm 3.1$ \\
\hline 93 & $\mathrm{C}_{8} \mathrm{H}_{18}$ & $2327.6 \pm 2.3$ & $2327.8 \pm 2.5$ & $2328.1 \pm 2.7$ & $2327.7 \pm 2.4$ & $2327.1 \pm 3.3$ & $2327.6 \pm 2.8$ & $2327.9 \pm 3.9$ & $2325.6 \pm 6.7$ & $2327.3 \pm 3.6$ \\
\hline 94 & $\mathrm{C}_{9} \mathrm{H}_{20}$ & $2604.6 \pm 2.6$ & $2604.8 \pm 2.8$ & $2605.2 \pm 3.0$ & $2604.7 \pm 2.7$ & $2604.0 \pm 3.7$ & $2604.6 \pm 3.1$ & $2604.9 \pm 4.4$ & $2602.4 \pm 7.5$ & $2604.2 \pm 4.0$ \\
\hline 95 & $\mathrm{C}_{10} \mathrm{H}_{2}$ & $1532.6 \pm 2.5$ & $1533.4 \pm 2.6$ & $1533.2 \pm 2.7$ & $1533.8 \pm 2.6$ & $1533.5 \pm 2.9$ & $1532.5 \pm 2.7$ & $1530.9 \pm 3.2$ & $1537.1 \pm 4.7$ & $1533.6 \pm 3.1$ \\
\hline 96 & $\mathrm{C}_{10} \mathrm{H}_{4}$ & $1662.4 \pm 2.5$ & $1662.9 \pm 2.5$ & $1662.6 \pm 2.6$ & $1663.4 \pm 2.5$ & $1663.1 \pm 2.9$ & $1662.5 \pm 2.7$ & $1662.6 \pm 3.3$ & $1664.7 \pm 5.0$ & $1663.4 \pm 3.1$ \\
\hline 97 & $\mathrm{C}_{10} \mathrm{H}_{8}$ & $2036.2 \pm 1.9$ & $2036.2 \pm 2.0$ & $2035.7 \pm 2.2$ & $2036.3 \pm 2.0$ & $2035.8 \pm 2.7$ & $2035.4 \pm 2.3$ & $2035.9 \pm 3.3$ & $2036.4 \pm 5.7$ & $2037.4 \pm 3.0$ \\
\hline 98 & $\mathrm{C}_{10} \mathrm{H}_{8}$ & $2072.4 \pm 2.2$ & $2072.4 \pm 2.3$ & $2071.7 \pm 2.4$ & $2072.2 \pm 2.3$ & $2071.6 \pm 3.0$ & $2071.3 \pm 2.6$ & $2071.4 \pm 3.5$ & $2072.0 \pm 5.9$ & $2074.2 \pm 3.2$ \\
\hline 99 & $\mathrm{C}_{10} \mathrm{H}_{8}$ & $2019.1 \pm 2.2$ & $2019.0 \pm 2.3$ & $2018.6 \pm 2.5$ & $2019.0 \pm 2.3$ & $2018.7 \pm 3.0$ & $2018.3 \pm 2.6$ & $2018.6 \pm 3.5$ & $2018.5 \pm 5.8$ & $2019.4 \pm 3.2$ \\
\hline 100 & $\mathrm{C}_{10} \mathrm{H}_{10}$ & $2069.2 \pm 1.9$ & $2069.2 \pm 2.1$ & $2068.8 \pm 2.2$ & $2069.3 \pm 2.0$ & $2069.1 \pm 2.8$ & $2069.0 \pm 2.4$ & $2068.8 \pm 3.4$ & $2071.2 \pm 5.8$ & $2069.6 \pm 3.0$ \\
\hline 101 & $\mathrm{C}_{10} \mathrm{H}_{10}$ & $2148.2 \pm 2.0$ & $2148.4 \pm 2.2$ & $2148.0 \pm 2.3$ & $2148.4 \pm 2.1$ & $2147.8 \pm 2.9$ & $2147.5 \pm 2.5$ & $2147.6 \pm 3.5$ & $2148.1 \pm 6.0$ & $2149.7 \pm 3.2$ \\
\hline 102 & $\mathrm{C}_{10} \mathrm{H}_{10}$ & $2139.1 \pm 2.0$ & $2139.2 \pm 2.1$ & $2138.6 \pm 2.3$ & $2139.1 \pm 2.1$ & $2138.8 \pm 2.9$ & $2138.7 \pm 2.4$ & $2138.8 \pm 3.5$ & $2139.0 \pm 6.0$ & $2140.3 \pm 3.1$ \\
\hline 103 & $\mathrm{C}_{10} \mathrm{H}_{10}$ & $2123.0 \pm 2.1$ & $2123.2 \pm 2.2$ & $2122.8 \pm 2.4$ & $2123.3 \pm 2.2$ & $2123.1 \pm 2.9$ & $2122.9 \pm 2.5$ & $2123.2 \pm 3.5$ & $2123.5 \pm 6.0$ & $2122.4 \pm 3.2$ \\
\hline 104 & $\mathrm{C}_{10} \mathrm{H}_{10}$ & $2098.5 \pm 2.0$ & $2098.5 \pm 2.1$ & $2098.5 \pm 2.3$ & $2098.5 \pm 2.1$ & $2098.9 \pm 2.8$ & $2098.9 \pm 2.4$ & $2098.5 \pm 3.4$ & $2099.3 \pm 5.9$ & $2098.1 \pm 3.1$ \\
\hline 105 & $\mathrm{C}_{10} \mathrm{H}_{10}$ & $2135.4 \pm 1.9$ & $2135.5 \pm 2.0$ & $2135.1 \pm 2.2$ & $2135.5 \pm 2.0$ & $2135.4 \pm 2.8$ & $2135.2 \pm 2.4$ & $2135.1 \pm 3.4$ & $2135.5 \pm 6.0$ & $2136.6 \pm 3.1$ \\
\hline 106 & $\mathrm{C}_{10} \mathrm{H}_{10}$ & $2127.1 \pm 2.0$ & $2127.2 \pm 2.1$ & $2126.7 \pm 2.3$ & $2127.3 \pm 2.1$ & $2127.2 \pm 2.9$ & $2127.2 \pm 2.4$ & $2127.5 \pm 3.5$ & $2127.4 \pm 6.0$ & $2127.3 \pm 3.1$ \\
\hline 107 & $\mathrm{C}_{10} \mathrm{H}_{10}$ & $2117.0 \pm 1.9$ & $2116.8 \pm 2.0$ & $2116.0 \pm 2.2$ & $2116.8 \pm 2.0$ & $2117.4 \pm 2.8$ & $2117.5 \pm 2.3$ & $2117.6 \pm 3.4$ & $2118.0 \pm 5.9$ & $2117.1 \pm 3.1$ \\
\hline 108 & $\mathrm{C}_{10} \mathrm{H}_{10}$ & $2090.7 \pm 2.1$ & $2091.1 \pm 2.2$ & $2091.0 \pm 2.4$ & $2091.5 \pm 2.2$ & $2091.0 \pm 2.9$ & $2090.7 \pm 2.5$ & $2090.4 \pm 3.5$ & $2092.3 \pm 5.9$ & $2091.2 \pm 3.2$ \\
\hline 109 & $\mathrm{C}_{10} \mathrm{H}_{10}$ & $2119.7 \pm 2.0$ & $2119.8 \pm 2.2$ & $2119.6 \pm 2.3$ & $2119.9 \pm 2.1$ & $2120.0 \pm 2.9$ & $2120.0 \pm 2.5$ & $2119.9 \pm 3.5$ & $2119.9 \pm 6.0$ & $2120.3 \pm 3.2$ \\
\hline 110 & $\mathrm{C}_{10} \mathrm{H}_{10}$ & $2134.0 \pm 1.9$ & $2133.9 \pm 2.0$ & $2133.3 \pm 2.2$ & $2134.0 \pm 2.0$ & $2134.3 \pm 2.8$ & $2134.4 \pm 2.4$ & $2134.4 \pm 3.4$ & $2134.6 \pm 6.0$ & $2134.4 \pm 3.1$ \\
\hline 111 & $\mathrm{C}_{10} \mathrm{H}_{10}$ & $2150.1 \pm 1.9$ & $2150.5 \pm 2.1$ & $2150.2 \pm 2.2$ & $2150.6 \pm 2.0$ & $2150.4 \pm 2.9$ & $2150.4 \pm 2.4$ & $2150.7 \pm 3.5$ & $2149.7 \pm 6.0$ & $2150.5 \pm 3.1$ \\
\hline 112 & $\mathrm{C}_{10} \mathrm{H}_{12}$ & $2246.3 \pm 2.3$ & $2246.4 \pm 2.4$ & $2246.4 \pm 2.6$ & $2246.4 \pm 2.4$ & $2245.8 \pm 3.2$ & $2245.8 \pm 2.7$ & $2246.2 \pm 3.8$ & $2245.3 \pm 6.4$ & $2245.9 \pm 3.4$ \\
\hline 113 & $\mathrm{C}_{10} \mathrm{H}_{16}$ & $2545.0 \pm 2.3$ & $2545.6 \pm 2.4$ & $2545.6 \pm 2.6$ & $2545.6 \pm 2.4$ & $2545.2 \pm 3.4$ & $2545.7 \pm 2.8$ & $2545.7 \pm 4.1$ & $2544.1 \pm 7.2$ & $2545.4 \pm 3.7$ \\
\hline 114 & $\mathrm{C}_{10} \mathrm{H}_{16}$ & $2536.1 \pm 2.3$ & $2536.6 \pm 2.4$ & $2536.7 \pm 2.6$ & $2536.5 \pm 2.4$ & $2536.2 \pm 3.4$ & $2536.7 \pm 2.8$ & $2536.8 \pm 4.1$ & $2535.4 \pm 7.1$ & $2536.2 \pm 3.7$ \\
\hline 115 & $\mathrm{C}_{10} \mathrm{H}_{18}$ & $2655.7 \pm 2.4$ & $2656.4 \pm 2.6$ & $2656.5 \pm 2.8$ & $2656.2 \pm 2.6$ & $2655.7 \pm 3.6$ & $2656.4 \pm 3.0$ & $2656.5 \pm 4.3$ & $2654.4 \pm 7.5$ & $2655.8 \pm 3.9$ \\
\hline
\end{tabular}


Table S9, contd.

\begin{tabular}{|c|c|c|c|c|c|c|c|c|c|c|}
\hline \multirow[t]{2}{*}{ MID } & & \multicolumn{9}{|c|}{ ATOMIC(hc) } \\
\hline & & $\mathrm{A}$ & $\mathrm{B}_{1}$ & $\mathrm{~B}_{2}$ & $\mathrm{~B}_{3}$ & $\mathrm{~B}_{4}$ & $\mathrm{~B}_{5}$ & $\mathrm{~B}_{6}$ & $\mathrm{C}$ & $\mathrm{E}_{\mathrm{CCSD}}$ \\
\hline 116 & $\mathrm{C}_{10} \mathrm{H}_{18}$ & $2658.8 \pm 2.4$ & $2659.3 \pm 2.6$ & $2659.4 \pm 2.8$ & $2659.2 \pm 2.6$ & $2658.7 \pm 3.6$ & $2659.3 \pm 3.0$ & $2659.5 \pm 4.3$ & $2657.2 \pm 7.5$ & $2658.8 \pm 3.9$ \\
\hline 117 & $\mathrm{C}_{10} \mathrm{H}_{22}$ & $2881.5 \pm 2.9$ & $2881.8 \pm 3.1$ & $2882.3 \pm 3.3$ & $2881.7 \pm 3.0$ & $2881.0 \pm 4.1$ & $2881.7 \pm 3.5$ & $2882.0 \pm 4.9$ & $2879.2 \pm 8.2$ & $2881.2 \pm 4.4$ \\
\hline 118 & $\mathrm{C}_{11} \mathrm{H}_{24}$ & $3158.5 \pm 3.2$ & $3158.9 \pm 3.3$ & $3159.3 \pm 3.6$ & $3158.7 \pm 3.3$ & $3157.9 \pm 4.4$ & $3158.7 \pm 3.8$ & $3159.1 \pm 5.3$ & $3155.9 \pm 9.0$ & $3158.1 \pm 4.8$ \\
\hline 119 & $\mathrm{C}_{12} \mathrm{H}_{6}$ & $2090.2 \pm 2.4$ & $2089.7 \pm 2.5$ & $2089.1 \pm 2.6$ & $2090.5 \pm 2.4$ & $2091.6 \pm 3.1$ & $2090.5 \pm 2.7$ & $2090.0 \pm 3.6$ & $2092.7 \pm 6.0$ & $2089.0 \pm 3.3$ \\
\hline 120 & $\mathrm{C}_{12} \mathrm{H}_{6}$ & $1993.4 \pm 2.2$ & $1993.7 \pm 2.3$ & $1992.7 \pm 2.4$ & $1993.5 \pm 2.2$ & $1994.3 \pm 2.9$ & $1993.2 \pm 2.5$ & $1993.6 \pm 3.4$ & $1995.2 \pm 5.6$ & $1993.9 \pm 3.1$ \\
\hline 121 & $\mathrm{C}_{12} \mathrm{H}_{8}$ & $2385.3 \pm 2.6$ & $2385.3 \pm 2.7$ & $2384.4 \pm 2.8$ & $2385.3 \pm 2.7$ & $2384.8 \pm 3.4$ & $2384.1 \pm 3.0$ & $2384.2 \pm 4.1$ & $2385.6 \pm 6.8$ & $2387.4 \pm 3.7$ \\
\hline 122 & $\mathrm{C}_{12} \mathrm{H}_{8}$ & $2348.1 \pm 2.5$ & $2348.1 \pm 2.6$ & $2347.4 \pm 2.8$ & $2348.0 \pm 2.6$ & $2347.7 \pm 3.3$ & $2347.0 \pm 2.9$ & $2346.8 \pm 4.0$ & $2348.7 \pm 6.6$ & $2350.1 \pm 3.6$ \\
\hline 123 & $\mathrm{C}_{12} \mathrm{H}_{10}$ & $2507.0 \pm 2.5$ & $2507.2 \pm 2.6$ & $2506.4 \pm 2.8$ & $2506.9 \pm 2.6$ & $2506.2 \pm 3.5$ & $2505.8 \pm 3.0$ & $2505.9 \pm 4.2$ & $2506.4 \pm 7.1$ & $2509.6 \pm 3.8$ \\
\hline 124 & $\mathrm{C}_{12} \mathrm{H}_{12}$ & & & & $2596.9 \pm 2.6$ & $2596.4 \pm 3.6$ & $2596.2 \pm 3.0$ & $2596.6 \pm 4.3$ & $2596.7 \pm 7.3$ & $2598.2 \pm 3.9$ \\
\hline 125 & $\mathrm{C}_{12} \mathrm{H}_{14}$ & $2675.4 \pm 2.8$ & $2675.6 \pm 2.9$ & $2675.5 \pm 3.1$ & $2675.6 \pm 2.9$ & $2674.9 \pm 3.8$ & $2674.9 \pm 3.3$ & $2675.4 \pm 4.5$ & $2674.4 \pm 7.6$ & $2674.9 \pm 4.1$ \\
\hline 126 & $\mathrm{C}_{12} \mathrm{H}_{18}$ & $2975.9 \pm 2.6$ & $2975.9 \pm 2.8$ & $2975.4 \pm 3.1$ & $2975.9 \pm 2.8$ & $2975.3 \pm 3.9$ & $2975.7 \pm 3.3$ & $2976.2 \pm 4.8$ & $2975.9 \pm 8.3$ & $2976.9 \pm 4.3$ \\
\hline 127 & $\mathrm{C}_{12} \mathrm{H}_{26}$ & $3435.4 \pm 3.4$ & $3435.9 \pm 3.6$ & $3436.4 \pm 3.9$ & $3435.7 \pm 3.6$ & $3434.9 \pm 4.8$ & $3435.7 \pm 4.1$ & $3436.1 \pm 5.8$ & $3432.7 \pm 9.8$ & $3435.1 \pm 5.2$ \\
\hline 128 & $\mathrm{C}_{13} \mathrm{H}_{10}$ & $2674.8 \pm 2.7$ & $2675.0 \pm 2.8$ & $2674.0 \pm 3.0$ & $2674.8 \pm 2.8$ & $2674.2 \pm 3.7$ & $2673.7 \pm 3.2$ & $2673.9 \pm 4.4$ & $2674.6 \pm 7.5$ & $2677.6 \pm 4.0$ \\
\hline 129 & $\mathrm{C}_{13} \mathrm{H}_{28}$ & $3712.4 \pm 3.6$ & $3712.9 \pm 3.9$ & $3713.4 \pm 4.2$ & $3712.7 \pm 3.8$ & $3711.8 \pm 5.2$ & $3712.7 \pm 4.4$ & $3713.1 \pm 6.2$ & $3709.5 \pm 10.6$ & $3712.0 \pm 5.6$ \\
\hline 130 & $\mathrm{C}_{14} \mathrm{H}_{8}$ & $2651.1 \pm 2.6$ & $2650.7 \pm 2.7$ & $2649.7 \pm 2.9$ & $2651.2 \pm 2.7$ & $2651.0 \pm 3.6$ & $2649.9 \pm 3.1$ & $2649.9 \pm 4.4$ & $2652.8 \pm 7.4$ & $2652.6 \pm 4.0$ \\
\hline 131 & $\mathrm{C}_{14} \mathrm{H}_{8}$ & $2684.9 \pm 3.0$ & $2684.8 \pm 3.1$ & $2683.8 \pm 3.3$ & $2685.0 \pm 3.1$ & $2684.6 \pm 3.9$ & $2683.6 \pm 3.4$ & $2683.7 \pm 4.6$ & $2686.3 \pm 7.6$ & $2686.7 \pm 4.2$ \\
\hline 132 & $\mathrm{C}_{14} \mathrm{H}_{10}$ & $2835.2 \pm 3.1$ & $2835.2 \pm 3.2$ & $2834.1 \pm 3.4$ & $2835.1 \pm 3.2$ & $2834.4 \pm 4.1$ & $2833.7 \pm 3.6$ & $2834.2 \pm 4.8$ & $2835.7 \pm 8.0$ & $2837.5 \pm 4.4$ \\
\hline 133 & $\mathrm{C}_{14} \mathrm{H}_{10}$ & $2840.9 \pm 3.1$ & $2841.0 \pm 3.2$ & $2839.9 \pm 3.4$ & $2840.8 \pm 3.2$ & $2840.0 \pm 4.1$ & $2839.3 \pm 3.6$ & $2839.7 \pm 4.8$ & $2841.5 \pm 8.1$ & $2843.7 \pm 4.4$ \\
\hline 134 & $\mathrm{C}_{14} \mathrm{H}_{20}$ & $3433.5 \pm 3.0$ & $3434.7 \pm 3.2$ & $3434.4 \pm 3.5$ & $3434.8 \pm 3.1$ & $3434.3 \pm 4.5$ & $3435.0 \pm 3.7$ & $3435.0 \pm 5.5$ & $3433.2 \pm 9.6$ & $3434.3 \pm 4.9$ \\
\hline 135 & $\mathrm{C}_{14} \mathrm{H}_{30}$ & $3989.4 \pm 3.9$ & $3989.9 \pm 4.2$ & $3990.5 \pm 4.5$ & $3989.7 \pm 4.1$ & $3988.7 \pm 5.6$ & $3989.7 \pm 4.7$ & $3990.2 \pm 6.7$ & $3986.3 \pm 11.4$ & $3988.9 \pm 6.0$ \\
\hline 136 & $\mathrm{C}_{15} \mathrm{H}_{32}$ & $4266.3 \pm 4.2$ & $4267.0 \pm 4.4$ & $4267.6 \pm 4.8$ & $4266.7 \pm 4.4$ & $4265.7 \pm 5.9$ & $4266.7 \pm 5.0$ & $4267.3 \pm 7.1$ & $4263.1 \pm 12.2$ & $4265.9 \pm 6.5$ \\
\hline 137 & $\mathrm{C}_{16} \mathrm{H}_{8}$ & $2932.9 \pm 3.2$ & $2932.9 \pm 3.3$ & $2931.7 \pm 3.5$ & $2933.3 \pm 3.3$ & $2933.7 \pm 4.2$ & $2932.3 \pm 3.7$ & $2931.8 \pm 5.0$ & $2934.8 \pm 8.3$ & $2934.3 \pm 4.6$ \\
\hline 138 & $\mathrm{C}_{16} \mathrm{H}_{10}$ & $3161.9 \pm 3.4$ & $3162.0 \pm 3.6$ & $3160.7 \pm 3.8$ & $3162.0 \pm 3.5$ & $3161.3 \pm 4.6$ & $3160.2 \pm 4.0$ & $3160.5 \pm 5.4$ & $3163.0 \pm 9.0$ & $3165.4 \pm 4.9$ \\
\hline 139 & $\mathrm{C}_{16} \mathrm{H}_{10}$ & $3176.0 \pm 3.5$ & $3175.9 \pm 3.6$ & $3174.5 \pm 3.8$ & $3175.9 \pm 3.6$ & $3175.1 \pm 4.6$ & $3174.0 \pm 4.0$ & $3174.6 \pm 5.4$ & $3177.6 \pm 9.0$ & $3179.1 \pm 5.0$ \\
\hline 140 & $\mathrm{C}_{16} \mathrm{H}_{12}$ & $3208.2 \pm 3.3$ & $3208.2 \pm 3.5$ & $3207.2 \pm 3.7$ & $3208.7 \pm 3.5$ & $3208.6 \pm 4.6$ & $3207.9 \pm 3.9$ & $3208.4 \pm 5.4$ & $3210.3 \pm 9.1$ & $3211.2 \pm 4.9$ \\
\hline 141 & $\mathrm{C}_{16} \mathrm{H}_{16}$ & $3493.6 \pm 3.3$ & $3494.0 \pm 3.5$ & $3493.1 \pm 3.8$ & $3494.1 \pm 3.5$ & $3493.6 \pm 4.8$ & $3493.5 \pm 4.0$ & $3493.6 \pm 5.7$ & $3494.6 \pm 9.8$ & $3496.9 \pm 5.2$ \\
\hline 142 & $\mathrm{C}_{16} \mathrm{H}_{16}$ & $3485.8 \pm 3.3$ & $3486.1 \pm 3.5$ & $3485.2 \pm 3.8$ & $3486.4 \pm 3.5$ & $3486.1 \pm 4.7$ & $3485.9 \pm 4.0$ & $3486.2 \pm 5.7$ & $3487.9 \pm 9.8$ & $3489.4 \pm 5.2$ \\
\hline 143 & $\mathrm{C}_{16} \mathrm{H}_{16}$ & $3475.3 \pm 3.4$ & $3475.5 \pm 3.6$ & $3474.6 \pm 3.8$ & $3475.8 \pm 3.5$ & $3475.5 \pm 4.8$ & $3475.3 \pm 4.1$ & $3475.5 \pm 5.7$ & $3477.1 \pm 9.8$ & $3478.5 \pm 5.2$ \\
\hline 144 & $\mathrm{C}_{16} \mathrm{H}_{34}$ & $4543.3 \pm 4.5$ & $4544.0 \pm 4.7$ & $4544.6 \pm 5.1$ & $4543.8 \pm 4.7$ & $4542.7 \pm 6.3$ & $4543.8 \pm 5.4$ & $4544.4 \pm 7.6$ & $4539.9 \pm 13.0$ & $4542.8 \pm 6.9$ \\
\hline
\end{tabular}


Table S9, contd.

\begin{tabular}{|c|c|c|c|c|c|c|c|c|c|c|}
\hline \multirow[t]{2}{*}{ MID } & & \multicolumn{9}{|c|}{$\operatorname{ATOMIC}(\mathrm{hc})$} \\
\hline & & $\mathrm{A}$ & $\mathrm{B}_{1}$ & $\mathrm{~B}_{2}$ & $\mathrm{~B}_{3}$ & $\mathrm{~B}_{4}$ & $\mathrm{~B}_{5}$ & $\mathrm{~B}_{6}$ & $\mathrm{C}$ & $\mathrm{E}_{\mathrm{CCSD}}$ \\
\hline 145 & $\mathrm{C}_{18} \mathrm{H}_{12}$ & $3602.1 \pm 3.8$ & $3602.3 \pm 4.0$ & $3600.9 \pm 4.2$ & $3602.2 \pm 3.9$ & $3601.3 \pm 5.1$ & $3600.2 \pm 4.4$ & $3600.8 \pm 6.1$ & $3603.6 \pm 10.2$ & $3605.8 \pm 5.5$ \\
\hline 146 & $\mathrm{C}_{18} \mathrm{H}_{12}$ & $3608.2 \pm 4.0$ & $3608.2 \pm 4.2$ & $3606.8 \pm 4.5$ & $3608.0 \pm 4.2$ & $3607.1 \pm 5.3$ & $3606.0 \pm 4.7$ & $3606.6 \pm 6.2$ & $3609.7 \pm 10.3$ & $3611.8 \pm 5.7$ \\
\hline 147 & $\mathrm{C}_{18} \mathrm{H}_{12}$ & $3596.4 \pm 4.0$ & $3596.5 \pm 4.1$ & $3595.0 \pm 4.4$ & $3596.5 \pm 4.1$ & $3595.7 \pm 5.3$ & $3594.6 \pm 4.6$ & $3595.4 \pm 6.2$ & $3598.0 \pm 10.2$ & $3599.1 \pm 5.7$ \\
\hline 148 & $\mathrm{C}_{18} \mathrm{H}_{12}$ & $3608.9 \pm 4.2$ & $3608.8 \pm 4.4$ & $3607.4 \pm 4.6$ & $3608.5 \pm 4.4$ & $3607.7 \pm 5.5$ & $3606.5 \pm 4.8$ & $3607.1 \pm 6.4$ & $3610.5 \pm 10.4$ & $3612.6 \pm 5.9$ \\
\hline 149 & $\mathrm{C}_{18} \mathrm{H}_{18}$ & $3946.7 \pm 3.6$ & $3947.0 \pm 3.8$ & $3945.8 \pm 4.2$ & $3947.1 \pm 3.8$ & $3946.3 \pm 5.3$ & $3945.8 \pm 4.4$ & $3946.7 \pm 6.4$ & $3948.2 \pm 11.0$ & $3949.6 \pm 5.7$ \\
\hline 150 & $\mathrm{C}_{18} \mathrm{H}_{18}$ & $3912.4 \pm 3.8$ & $3912.8 \pm 4.0$ & $3911.9 \pm 4.3$ & $3912.6 \pm 4.0$ & $3911.7 \pm 5.4$ & $3911.5 \pm 4.6$ & $3911.9 \pm 6.5$ & $3912.7 \pm 11.0$ & $3915.2 \pm 5.9$ \\
\hline 151 & $\mathrm{C}_{18} \mathrm{H}_{18}$ & $3945.5 \pm 3.7$ & $3945.7 \pm 3.9$ & $3944.5 \pm 4.2$ & $3945.8 \pm 3.8$ & $3945.0 \pm 5.3$ & $3944.6 \pm 4.5$ & $3945.4 \pm 6.4$ & $3947.0 \pm 11.0$ & $3948.4 \pm 5.8$ \\
\hline 152 & $\mathrm{C}_{20}$ & $2837.4 \pm 4.1$ & $2835.2 \pm 4.2$ & $2833.7 \pm 4.4$ & $2838.4 \pm 4.2$ & $2842.4 \pm 4.9$ & $2838.6 \pm 4.5$ & $2837.3 \pm 5.5$ & $2843.9 \pm 8.4$ & $2838.2 \pm 5.2$ \\
\hline 153 & $\mathrm{C}_{20} \mathrm{H}_{10}$ & $3794.5 \pm 4.1$ & $3795.1 \pm 4.3$ & $3793.1 \pm 4.6$ & $3795.3 \pm 4.3$ & $3794.7 \pm 5.5$ & $3793.0 \pm 4.8$ & $3793.2 \pm 6.5$ & $3796.9 \pm 10.7$ & $3798.8 \pm 5.9$ \\
\hline 154 & $\mathrm{C}_{20} \mathrm{H}_{12}$ & $3937.1 \pm 4.5$ & $3937.1 \pm 4.6$ & $3935.4 \pm 4.9$ & $3937.0 \pm 4.6$ & $3936.1 \pm 5.9$ & $3934.7 \pm 5.1$ & $3935.5 \pm 6.8$ & $3940.0 \pm 11.2$ & $3940.9 \pm 6.3$ \\
\hline 155 & $\mathrm{C}_{20} \mathrm{H}_{14}$ & $4035.4 \pm 4.1$ & $4035.8 \pm 4.3$ & $4034.1 \pm 4.6$ & $4035.8 \pm 4.3$ & $4035.2 \pm 5.7$ & $4034.1 \pm 4.9$ & $4034.1 \pm 6.7$ & $4036.0 \pm 11.4$ & $4040.0 \pm 6.1$ \\
\hline 156 & $\mathrm{C}_{20} \mathrm{H}_{20}$ & $4390.2 \pm 4.1$ & $4392.6 \pm 4.4$ & $4392.0 \pm 4.7$ & $4392.9 \pm 4.3$ & $4392.6 \pm 5.9$ & $4392.7 \pm 5.0$ & $4393.0 \pm 7.2$ & $4390.7 \pm 12.3$ & $4390.3 \pm 6.5$ \\
\hline 157 & $\mathrm{C}_{20} \mathrm{H}_{20}$ & $4362.8 \pm 4.0$ & $4363.8 \pm 4.3$ & $4363.1 \pm 4.6$ & $4364.7 \pm 4.2$ & $4365.1 \pm 5.9$ & $4365.0 \pm 4.9$ & $4364.6 \pm 7.1$ & $4366.7 \pm 12.2$ & $4364.0 \pm 6.4$ \\
\hline 158 & $\mathrm{C}_{20} \mathrm{H}_{36}$ & & & & $5222.8 \pm 4.8$ & $5222.9 \pm 6.8$ & $5223.7 \pm 5.7$ & $5224.0 \pm 8.4$ & $5225.5 \pm 14.7$ & $5221.3 \pm 7.5$ \\
\hline 159 & $\mathrm{C}_{24} \mathrm{H}_{12}$ & $4622.4 \pm 5.1$ & $4622.2 \pm 5.3$ & $4619.8 \pm 5.6$ & $4622.2 \pm 5.3$ & $4621.1 \pm 6.8$ & $4619.1 \pm 5.9$ & $4620.1 \pm 7.9$ & $4626.6 \pm 13.1$ & $4627.6 \pm 7.3$ \\
\hline 160 & $\mathrm{C}_{28} \mathrm{H}_{14}$ & & & & $5339.9 \pm 6.0$ & $5339.1 \pm 7.7$ & $5336.6 \pm 6.7$ & $5337.9 \pm 9.1$ & $5345.3 \pm 15.1$ & $5345.3 \pm 8.3$ \\
\hline 161 & $\mathrm{C}_{32} \mathrm{H}_{16}$ & & & & $6064.1 \pm 6.7$ & $6063.5 \pm 8.7$ & $6060.6 \pm 7.5$ & $6062.2 \pm 10.3$ & $6070.3 \pm 17.1$ & $6069.8 \pm 9.4$ \\
\hline
\end{tabular}

Molecules are identified by their molecule index MID as specified in Table S1. In analogy to Eqs. (3), (6), (9), and (10) of the paper, ZPE-inclusive atomization energies (subscript "A,0" for atomization energy ("A") at $T=0 \mathrm{~K}\left(\right.$ (")")) are defined as $E_{A, 0}^{\mathrm{ATOMIC}(\mathrm{hc})}[M]=$ $E_{A, 0}^{\mathrm{ATOMIC}}[M]+C_{A, 0}^{\mathrm{ATOMIC}(\mathrm{hc})}[M]+u_{A, 0}^{\mathrm{ATOMIC}(\mathrm{hc})}[M]$ with contributions

$$
\begin{aligned}
E_{A, 0}^{\mathrm{ATOMIC}}[M] & =E_{A, e}^{\mathrm{ATOMIC}}[M]-\mathrm{ZPE}[M] \\
C_{A, 0}^{\mathrm{ATOMIC}(\mathrm{hc})}[M] & =C_{A, e}^{\mathrm{ATOMIC}(\mathrm{hc})}[M]+C^{\mathrm{geo}}[M]-C^{\mathrm{ZPE}}[M] \\
\left(u_{A, 0}^{\mathrm{ATOMIC}(\mathrm{hc})}[M]\right)^{2} & =\left(u_{A, e}^{\mathrm{ATOMIC}(\mathrm{hc})}[M]\right)^{2}+\left(u^{\text {geo }}[M]\right)^{2}+\left(u^{\mathrm{ZPE}}[M]\right)^{2}
\end{aligned}
$$


Table S10: Best available theoretical data (in $\mathrm{kcal} / \mathrm{mol})^{a-c}$

\begin{tabular}{|c|c|c|c|c|c|c|}
\hline MID & & Molecule & $\Delta H_{f}^{0}[M, 298.15 K]$ & $\Delta H_{f}^{0}[M, 0 K]$ & $E_{A, 0}[M]$ & Note \\
\hline 1 & $\mathrm{CH}_{4}$ & methane & $-17.7 \pm 0.4$ & $-15.8 \pm 0.4$ & $392.4 \pm 0.4$ & III \\
\hline 2 & $\mathrm{C}_{2} \mathrm{H}_{2}$ & acetylene & $54.7 \pm 0.5$ & $54.8 \pm 0.5$ & $388.5 \pm 0.5$ & III \\
\hline 3 & $\mathrm{C}_{2} \mathrm{H}_{4}$ & ethylene & $12.6 \pm 0.3$ & $14.6 \pm 0.3$ & $532.0 \pm 0.3$ & III \\
\hline 4 & $\mathrm{C}_{2} \mathrm{H}_{6}$ & ethane & $-20.1 \pm 0.7$ & $-16.3 \pm 0.7$ & $666.2 \pm 0.7$ & III \\
\hline 5 & $\mathrm{C}_{3} \mathrm{H}_{4}$ & allene & $45.5 \pm 0.4$ & $47.3 \pm 0.3$ & $669.3 \pm 0.3$ & III \\
\hline 6 & $\mathrm{C}_{3} \mathrm{H}_{4}$ & cyclopropene & $68.0 \pm 0.4$ & $70.1 \pm 0.4$ & $646.5 \pm 0.4$ & III \\
\hline 7 & $\mathrm{C}_{3} \mathrm{H}_{4}$ & propyne & $44.5 \pm 0.5$ & $46.2 \pm 0.4$ & $670.5 \pm 0.4$ & III \\
\hline 8 & $\mathrm{C}_{3} \mathrm{H}_{6}$ & cyclopropane & $13.1 \pm 0.7$ & $17.2 \pm 0.7$ & $802.7 \pm 0.7$ & III \\
\hline 9 & $\mathrm{C}_{3} \mathrm{H}_{6}$ & propene & $4.9 \pm 0.6$ & $8.5 \pm 0.5$ & $811.4 \pm 0.5$ & III \\
\hline 10 & $\mathrm{C}_{3} \mathrm{H}_{8}$ & propane & $-25.1 \pm 0.9$ & $-19.7 \pm 0.8$ & $942.9 \pm 0.8$ & III \\
\hline 11 & $\mathrm{C}_{4} \mathrm{H}_{2}$ & 1,3-butadiyne & $110.1 \pm 0.9$ & $109.7 \pm 0.9$ & $673.7 \pm 0.9$ & III \\
\hline 12 & $\mathrm{C}_{4} \mathrm{H}_{4}$ & bicyclo[1.1.0]but-1(3)-ene & $136.6 \pm 0.7$ & $138.7 \pm 0.7$ & $748.0 \pm 0.7$ & III \\
\hline 13 & $\mathrm{C}_{4} \mathrm{H}_{4}$ & butatriene & $77.3 \pm 0.6$ & $78.7 \pm 0.5$ & $807.9 \pm 0.5$ & III \\
\hline 14 & $\mathrm{C}_{4} \mathrm{H}_{4}$ & cyclobutadiene & $102.5 \pm 0.6$ & $104.5 \pm 0.6$ & $782.2 \pm 0.6$ & III \\
\hline 15 & $\mathrm{C}_{4} \mathrm{H}_{4}$ & ethynylethene & $69.5 \pm 0.7$ & $71.1 \pm 0.7$ & $815.6 \pm 0.7$ & III \\
\hline 16 & $\mathrm{C}_{4} \mathrm{H}_{4}$ & methylenecyclopropene & $93.0 \pm 0.5$ & $94.9 \pm 0.4$ & $791.8 \pm 0.4$ & III \\
\hline 17 & $\mathrm{C}_{4} \mathrm{H}_{4}$ & tetrahedrane & $128.9 \pm 0.6$ & $130.9 \pm 0.6$ & $755.7 \pm 0.6$ & III \\
\hline 18 & $\mathrm{C}_{4} \mathrm{H}_{6}$ & 1,2-butadiene & $39.1 \pm 0.6$ & $42.3 \pm 0.5$ & $947.6 \pm 0.5$ & III \\
\hline 19 & $\mathrm{C}_{4} \mathrm{H}_{6}$ & 1,3-butadiene & $26.8 \pm 0.6$ & $30.3 \pm 0.6$ & $959.7 \pm 0.6$ & III \\
\hline 20 & $\mathrm{C}_{4} \mathrm{H}_{6}$ & 2-butyne & $35.3 \pm 0.6$ & $38.4 \pm 0.5$ & $951.5 \pm 0.5$ & III \\
\hline 21 & $\mathrm{C}_{4} \mathrm{H}_{6}$ & bicyclo[1.1.0]butane & $54.0 \pm 0.6$ & $58.1 \pm 0.6$ & $931.8 \pm 0.6$ & III \\
\hline 22 & $\mathrm{C}_{4} \mathrm{H}_{6}$ & cyclobutene & $38.7 \pm 0.5$ & $42.7 \pm 0.5$ & $947.2 \pm 0.5$ & III \\
\hline 23 & $\mathrm{C}_{4} \mathrm{H}_{6}$ & methylenecyclopropane & $46.5 \pm 0.6$ & $50.3 \pm 0.6$ & $939.7 \pm 0.6$ & III \\
\hline 24 & $\mathrm{C}_{4} \mathrm{H}_{8}$ & cyclobutane & $6.6 \pm 0.9$ & $12.5 \pm 0.9$ & $1080.7 \pm 0.9$ & III \\
\hline 25 & $\mathrm{C}_{4} \mathrm{H}_{8}$ & isobutene & $-3.8 \pm 0.7$ & $1.3 \pm 0.7$ & $1091.9 \pm 0.7$ & III \\
\hline 26 & $\mathrm{C}_{4} \mathrm{H}_{10}$ & isobutane & $-31.9 \pm 1.0$ & $-25.0 \pm 1.0$ & $1221.5 \pm 1.0$ & II \\
\hline 27 & $\mathrm{C}_{4} \mathrm{H}_{10}$ & $n$-butane & $-29.8 \pm 1.1$ & $-23.3 \pm 1.0$ & $1219.7 \pm 1.0$ & II \\
\hline 28 & $\mathrm{C}_{5} \mathrm{H}_{4}$ & 1,4-pentadiyne & $109.1 \pm 0.9$ & $110.2 \pm 0.8$ & $946.5 \pm 0.8$ & III \\
\hline 29 & $\mathrm{C}_{5} \mathrm{H}_{4}$ & pentatetraene & $107.3 \pm 0.7$ & $108.4 \pm 0.6$ & $948.3 \pm 0.6$ & III \\
\hline
\end{tabular}


Table S10, contd.

\begin{tabular}{|c|c|c|c|c|c|c|}
\hline MID & $\mathrm{D}$ & Molecule & $\Delta H_{f}^{0}[M, 298.15 K]$ & $\Delta H_{f}^{0}[M, 0 K]$ & $E_{A, 0}[M]$ & Note \\
\hline 30 & $\mathrm{C}_{5} \mathrm{H}_{4}$ & spiropentadiene & $154.3 \pm 0.6$ & $156.0 \pm 0.6$ & $900.7 \pm 0.6$ & III \\
\hline 31 & $\mathrm{C}_{5} \mathrm{H}_{6}$ & [1.1.1]propellane & $87.3 \pm 0.7$ & $91.5 \pm 0.7$ & $1068.4 \pm 0.7$ & II \\
\hline 32 & $\mathrm{C}_{5} \mathrm{H}_{6}$ & 1,3-cyclopentadiene & $32.1 \pm 0.7$ & $36.1 \pm 0.7$ & $1123.8 \pm 0.7$ & II \\
\hline 33 & $\mathrm{C}_{5} \mathrm{H}_{6}$ & 3-methylenecyclobutene & $60.2 \pm 0.8$ & $63.9 \pm 0.7$ & $1096.0 \pm 0.7$ & I \\
\hline 34 & $\mathrm{C}_{5} \mathrm{H}_{6}$ & bicyclo[2.1.0]pent-2-ene & $79.0 \pm 0.6$ & $83.1 \pm 0.6$ & $1076.9 \pm 0.6$ & II \\
\hline 35 & $\mathrm{C}_{5} \mathrm{H}_{6}$ & cyclopropylacetylene & $71.4 \pm 0.7$ & $74.8 \pm 0.7$ & $1085.2 \pm 0.7$ & I \\
\hline 36 & $\mathrm{C}_{5} \mathrm{H}_{8}$ & 1,4-pentadiene & $25.7 \pm 0.7$ & $30.6 \pm 0.7$ & $1232.6 \pm 0.7$ & II \\
\hline 37 & $\mathrm{C}_{5} \mathrm{H}_{8}$ & isoprene & $18.5 \pm 0.8$ & $23.5 \pm 0.7$ & $1239.7 \pm 0.7$ & II \\
\hline 38 & $\mathrm{C}_{5} \mathrm{H}_{8}$ & spiropentane & $44.7 \pm 0.9$ & $50.3 \pm 0.9$ & $1212.9 \pm 0.9$ & II \\
\hline 39 & $\mathrm{C}_{5} \mathrm{H}_{10}$ & cyclopentane & $-18.1 \pm 1.1$ & $-10.4 \pm 1.0$ & $1376.8 \pm 1.0$ & II \\
\hline 40 & $\mathrm{C}_{5} \mathrm{H}_{12}$ & neopentane & $-39.9 \pm 1.1$ & $-31.5 \pm 1.1$ & $1501.2 \pm 1.1$ & II \\
\hline 41 & $\mathrm{C}_{5} \mathrm{H}_{12}$ & $n$-pentane & $-34.5 \pm 1.3$ & $-26.9 \pm 1.2$ & $1496.6 \pm 1.2$ & II \\
\hline 42 & $\mathrm{C}_{6} \mathrm{H}_{2}$ & 1,3,5-hexatriyne & $164.2 \pm 1.2$ & $163.0 \pm 1.2$ & $960.4 \pm 1.2$ & III \\
\hline 43 & $\mathrm{C}_{6} \mathrm{H}_{4}$ & butalene & $169.5 \pm 0.8$ & $171.4 \pm 0.8$ & $1055.3 \pm 0.8$ & III \\
\hline 44 & $\mathrm{C}_{6} \mathrm{H}_{4}$ & cis-3-hexene-1,5-diyne & $126.3 \pm 1.2$ & $127.2 \pm 1.1$ & $1099.5 \pm 1.1$ & II \\
\hline 45 & $\mathrm{C}_{6} \mathrm{H}_{4}$ & hexapentaene & $137.0 \pm 0.8$ & $137.6 \pm 0.8$ & $1089.1 \pm 0.8$ & III \\
\hline 46 & $\mathrm{C}_{6} \mathrm{H}_{4}$ & ortho-benzyne & $110.1 \pm 0.6$ & $112.3 \pm 0.6$ & $1114.4 \pm 0.6$ & II \\
\hline 47 & $\mathrm{C}_{6} \mathrm{H}_{4}$ & trans-3-hexene-1,5-diyne & $126.3 \pm 1.3$ & $127.1 \pm 1.3$ & $1099.6 \pm 1.3$ & I \\
\hline 48 & $\mathrm{C}_{6} \mathrm{H}_{4}$ & triafulvalene & $185.1 \pm 0.8$ & $186.2 \pm 0.8$ & $1040.5 \pm 0.8$ & III \\
\hline 49 & $\mathrm{C}_{6} \mathrm{H}_{6}$ & 2,4-hexadiyne & $88.7 \pm 0.8$ & $91.0 \pm 0.7$ & $1239.0 \pm 0.7$ & II \\
\hline 50 & $\mathrm{C}_{6} \mathrm{H}_{6}$ & 3,3'-bicyclopropenyl & $140.5 \pm 0.8$ & $143.6 \pm 0.8$ & $1186.4 \pm 0.8$ & II \\
\hline 51 & $\mathrm{C}_{6} \mathrm{H}_{6}$ & 3,4-dimethylenecyclobutene & $81.5 \pm 0.9$ & $84.9 \pm 0.9$ & $1245.1 \pm 0.9$ & II \\
\hline 52 & $\mathrm{C}_{6} \mathrm{H}_{6}$ & [3]radialene & $106.1 \pm 0.8$ & $109.1 \pm 0.8$ & $1220.9 \pm 0.8$ & II \\
\hline 53 & $\mathrm{C}_{6} \mathrm{H}_{6}$ & benzene & $20.1 \pm 0.9$ & $24.2 \pm 0.9$ & $1305.8 \pm 0.9$ & III \\
\hline 54 & $\mathrm{C}_{6} \mathrm{H}_{6}$ & benzvalene & $92.9 \pm 0.8$ & $97.1 \pm 0.8$ & $1232.9 \pm 0.8$ & II \\
\hline 55 & $\mathrm{C}_{6} \mathrm{H}_{6}$ & Dewar benzene & $96.7 \pm 0.8$ & $100.8 \pm 0.8$ & $1229.2 \pm 0.8$ & II \\
\hline 56 & $\mathrm{C}_{6} \mathrm{H}_{6}$ & fulvene & $51.5 \pm 1.0$ & $55.3 \pm 0.9$ & $1274.7 \pm 0.9$ & II \\
\hline 57 & $\mathrm{C}_{6} \mathrm{H}_{6}$ & prismane & $133.5 \pm 0.8$ & $137.8 \pm 0.8$ & $1192.1 \pm 0.8$ & II \\
\hline 58 & $\mathrm{C}_{6} \mathrm{H}_{8}$ & 1,3-cyclohexadiene & $25.9 \pm 0.9$ & $31.6 \pm 0.8$ & $1401.6 \pm 0.8$ & II \\
\hline 59 & $\mathrm{C}_{6} \mathrm{H}_{8}$ & 1,4-cyclohexadiene & $26.0 \pm 0.8$ & $31.6 \pm 0.8$ & $1401.7 \pm 0.8$ & II \\
\hline
\end{tabular}


Table S10, contd.

\begin{tabular}{|c|c|c|c|c|c|c|}
\hline MID & & Molecule & $\Delta H_{f}^{0}[M, 298.15 K]$ & $\Delta H_{f}^{0}[M, 0 K]$ & $E_{A, 0}[M]$ & Note \\
\hline 60 & $\mathrm{C}_{6} \mathrm{H}_{8}$ & trans-1,3,5-hexatriene & $40.1 \pm 1.0$ & $44.8 \pm 1.0$ & $1388.5 \pm 1.0$ & II \\
\hline 61 & $\mathrm{C}_{6} \mathrm{H}_{10}$ & cyclohexene & $-0.7 \pm 1.0$ & $6.8 \pm 1.0$ & $1529.7 \pm 1.0$ & II \\
\hline 62 & $\mathrm{C}_{6} \mathrm{H}_{12}$ & cyclohexane & $-29.0 \pm 1.2$ & $-19.6 \pm 1.2$ & $1659.4 \pm 1.2$ & II \\
\hline 63 & $\mathrm{C}_{6} \mathrm{H}_{14}$ & 3-methylpentane & $-40.2 \pm 1.4$ & $-31.0 \pm 1.4$ & $1774.1 \pm 1.4$ & I \\
\hline 64 & $\mathrm{C}_{6} \mathrm{H}_{14}$ & $n$-hexane & $-39.3 \pm 1.5$ & $-30.5 \pm 1.4$ & $1773.5 \pm 1.4$ & I \\
\hline 65 & $\mathrm{C}_{7} \mathrm{H}_{4}$ & 3,3-diethynylcyclopropene & $185.8 \pm 1.2$ & $186.5 \pm 1.2$ & $1210.3 \pm 1.2$ & I \\
\hline 66 & $\mathrm{C}_{7} \mathrm{H}_{4}$ & heptahexaene & $165.9 \pm 1.0$ & $166.1 \pm 0.9$ & $1230.6 \pm 0.9$ & II \\
\hline 67 & $\mathrm{C}_{7} \mathrm{H}_{6}$ & 1,1-diethynylcyclopropane & $131.9 \pm 1.1$ & $134.5 \pm 1.1$ & $1365.5 \pm 1.1$ & I \\
\hline 68 & $\mathrm{C}_{7} \mathrm{H}_{6}$ & 1,3,5-norcaratriene & $91.5 \pm 0.9$ & $95.3 \pm 0.9$ & $1404.7 \pm 0.8$ & II \\
\hline 69 & $\mathrm{C}_{7} \mathrm{H}_{6}$ & fulvenallene & $84.8 \pm 1.0$ & $88.1 \pm 1.0$ & $1411.9 \pm 1.0$ & II \\
\hline 70 & $\mathrm{C}_{7} \mathrm{H}_{6}$ & spiro[2.4]hepta-1,4,6-triene & $106.7 \pm 1.0$ & $110.3 \pm 1.0$ & $1389.8 \pm 1.0$ & II \\
\hline 71 & $\mathrm{C}_{7} \mathrm{H}_{8}$ & 1,3,5-cycloheptatriene & $44.9 \pm 1.2$ & $50.4 \pm 1.2$ & $1552.8 \pm 1.2$ & I \\
\hline 72 & $\mathrm{C}_{7} \mathrm{H}_{8}$ & 2,5-norbornadiene & $57.9 \pm 1.0$ & $64.0 \pm 1.0$ & $1539.3 \pm 1.0$ & II \\
\hline 73 & $\mathrm{C}_{7} \mathrm{H}_{8}$ & norcaradiene & $50.6 \pm 1.1$ & $56.3 \pm 1.1$ & $1547.0 \pm 1.1$ & II \\
\hline 74 & $\mathrm{C}_{7} \mathrm{H}_{8}$ & quadricyclane & $80.5 \pm 0.9$ & $86.8 \pm 0.9$ & $1516.5 \pm 0.9$ & II \\
\hline 75 & $\mathrm{C}_{7} \mathrm{H}_{8}$ & spiro[2.4]hepta-4,6-diene & $54.7 \pm 1.0$ & $60.2 \pm 1.0$ & $1543.1 \pm 1.0$ & II \\
\hline 76 & $\mathrm{C}_{7} \mathrm{H}_{8}$ & toluene & $12.1 \pm 1.1$ & $17.6 \pm 1.1$ & $1585.7 \pm 1.1$ & I \\
\hline 77 & $\mathrm{C}_{7} \mathrm{H}_{12}$ & norbornane & $-13.0 \pm 1.2$ & $-3.4 \pm 1.2$ & $1813.2 \pm 1.2$ & I \\
\hline 78 & $\mathrm{C}_{7} \mathrm{H}_{16}$ & $n$-heptane & $-44.1 \pm 1.7$ & $-34.2 \pm 1.6$ & $2050.5 \pm 1.6$ & I \\
\hline 79 & $\mathrm{C}_{8} \mathrm{H}_{2}$ & 1,3,5,7-octatetrayne & $218.0 \pm 1.6$ & $216.0 \pm 1.6$ & $1247.5 \pm 1.6$ & II \\
\hline 80 & $\mathrm{C}_{8} \mathrm{H}_{4}$ & 1,2,3,5,6,7-cyclooctahexaene & $199.1 \pm 1.2$ & $200.3 \pm 1.2$ & $1366.5 \pm 1.2$ & II \\
\hline 81 & $\mathrm{C}_{8} \mathrm{H}_{4}$ & 1,5-cyclooctadiene-3,7-diyne & $174.9 \pm 1.2$ & $176.3 \pm 1.2$ & $1390.5 \pm 1.2$ & II \\
\hline 82 & $\mathrm{C}_{8} \mathrm{H}_{4}$ & octaheptaene & $194.6 \pm 1.1$ & $194.4 \pm 1.1$ & $1372.3 \pm 1.1$ & II \\
\hline 83 & $\mathrm{C}_{8} \mathrm{H}_{6}$ & benzocyclobutadiene & $98.2 \pm 1.4$ & $101.9 \pm 1.4$ & $1568.1 \pm 1.4$ & I \\
\hline 84 & $\mathrm{C}_{8} \mathrm{H}_{6}$ & bis(cyclopropa)benzene & $160.4 \pm 0.9$ & $163.9 \pm 0.8$ & $1506.1 \pm 0.8$ & II \\
\hline 85 & $\mathrm{C}_{8} \mathrm{H}_{6}$ & pentalene & $89.2 \pm 1.3$ & $93.0 \pm 1.2$ & $1577.1 \pm 1.2$ & II \\
\hline 86 & $\mathrm{C}_{8} \mathrm{H}_{8}$ & 1,3,5,7-cyclooctatetraene & $72.0 \pm 1.3$ & $77.1 \pm 1.3$ & $1696.2 \pm 1.3$ & II \\
\hline 87 & $\mathrm{C}_{8} \mathrm{H}_{8}$ & benzocyclobutene & $48.2 \pm 1.1$ & $53.8 \pm 1.1$ & $1719.5 \pm 1.1$ & II \\
\hline 88 & $\mathrm{C}_{8} \mathrm{H}_{8}$ & cubane & $145.5 \pm 1.2$ & $152.0 \pm 1.2$ & $1621.3 \pm 1.2$ & II \\
\hline 89 & $\mathrm{C}_{8} \mathrm{H}_{8}$ & para-xylylene & $53.0 \pm 1.4$ & $58.1 \pm 1.4$ & $1715.2 \pm 1.4$ & II \\
\hline
\end{tabular}


Table S10, contd.

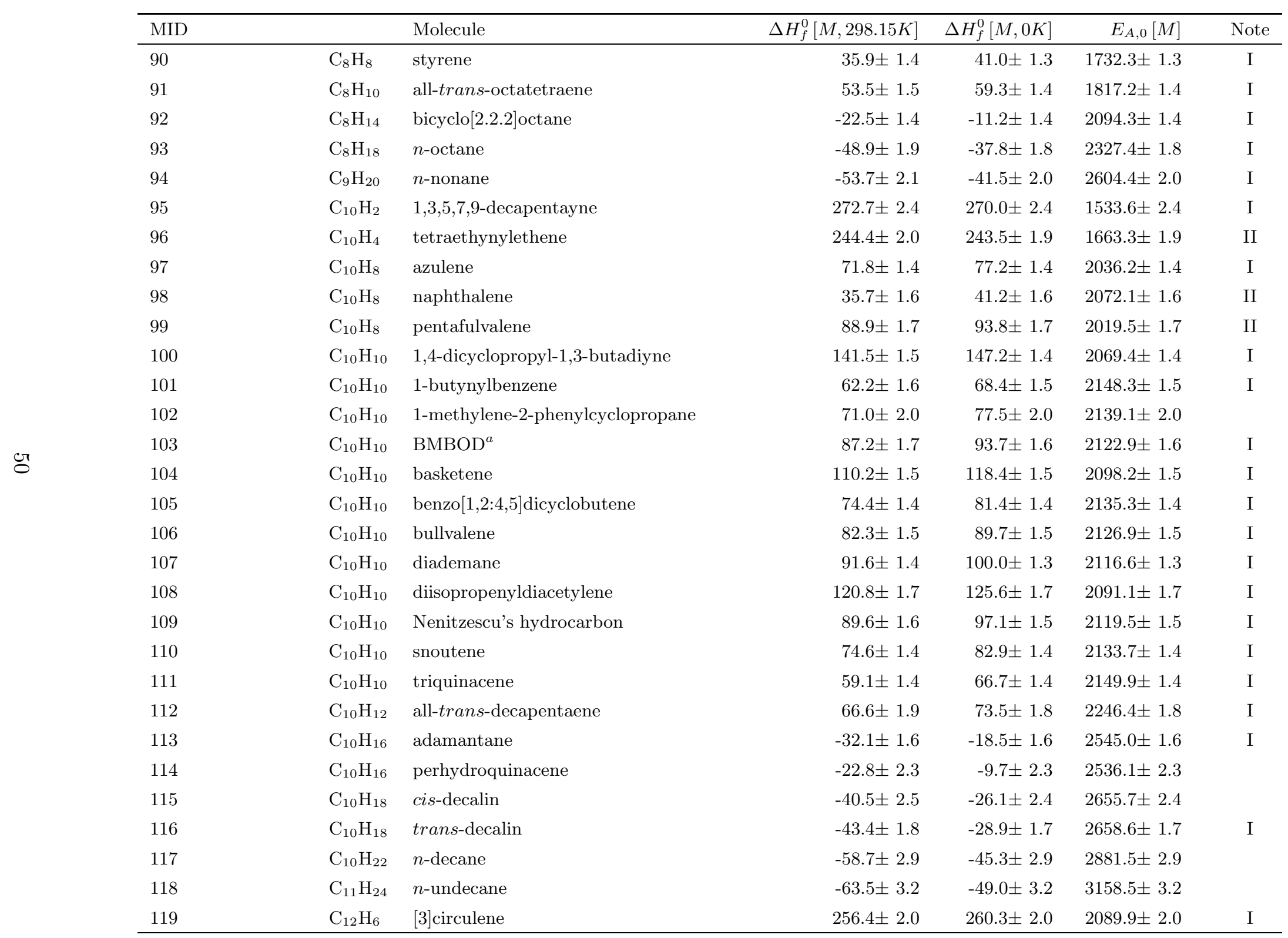


Table S10, contd.

\begin{tabular}{|c|c|c|c|c|c|c|c|}
\hline & MID & & Molecule & $\Delta H_{f}^{0}[M, 298.15 K]$ & $\Delta H_{f}^{0}[M, 0 K]$ & $E_{A, 0}[M]$ & Note \\
\hline & 120 & $\mathrm{C}_{12} \mathrm{H}_{6}$ & tricyclopropenylidenecyclopropane & $355.8 \pm 1.8$ & $356.8 \pm 1.8$ & $1993.4 \pm 1.8$ & I \\
\hline & 121 & $\mathrm{C}_{12} \mathrm{H}_{8}$ & acenaphthylene & $62.7 \pm 2.1$ & $68.1 \pm 2.1$ & $2385.3 \pm 2.1$ & I \\
\hline & 122 & $\mathrm{C}_{12} \mathrm{H}_{8}$ & biphenylene & $100.2 \pm 2.0$ & $105.3 \pm 2.0$ & $2348.1 \pm 2.0$ & I \\
\hline & 123 & $\mathrm{C}_{12} \mathrm{H}_{10}$ & biphenyl & $43.0 \pm 2.0$ & $49.7 \pm 2.0$ & $2507.0 \pm 2.0$ & $\mathrm{I}$ \\
\hline & 124 & $\mathrm{C}_{12} \mathrm{H}_{12}$ & 1,2-benzo-1,3,7-cyclooctatriene & $54.6 \pm 2.6$ & $63.0 \pm 2.6$ & $2596.9 \pm 2.6$ & $\mathrm{~B}_{3}$ \\
\hline & 125 & $\mathrm{C}_{12} \mathrm{H}_{14}$ & all-trans-dodecahexaene & $79.7 \pm 2.2$ & $87.7 \pm 2.2$ & $2675.5 \pm 2.2$ & I \\
\hline & 126 & $\mathrm{C}_{12} \mathrm{H}_{18}$ & hexamethylbenzene & $-17.6 \pm 2.7$ & $-6.1 \pm 2.6$ & $2975.9 \pm 2.6$ & \\
\hline & 127 & $\mathrm{C}_{12} \mathrm{H}_{26}$ & $n$-dodecane & $-68.3 \pm 3.5$ & $-52.6 \pm 3.4$ & $3435.4 \pm 3.4$ & \\
\hline & 128 & $\mathrm{C}_{13} \mathrm{H}_{10}$ & fluorene & $45.0 \pm 2.7$ & $51.9 \pm 2.7$ & $2674.8 \pm 2.7$ & \\
\hline & 129 & $\mathrm{C}_{13} \mathrm{H}_{28}$ & $n$-tridecane & $-73.1 \pm 3.7$ & $-56.3 \pm 3.7$ & $3712.4 \pm 3.6$ & \\
\hline & 130 & $\mathrm{C}_{14} \mathrm{H}_{8}$ & 9,10-didehydrophenanthrene & $137.4 \pm 2.6$ & $142.4 \pm 2.6$ & $2651.1 \pm 2.6$ & \\
\hline & 131 & $\mathrm{C}_{14} \mathrm{H}_{8}$ & pyracyclene & $103.4 \pm 2.5$ & $108.6 \pm 2.5$ & $2684.9 \pm 2.5$ & I \\
\hline & 132 & $\mathrm{C}_{14} \mathrm{H}_{10}$ & anthracene & $54.7 \pm 2.5$ & $61.5 \pm 2.5$ & $2835.2 \pm 2.5$ & $\mathrm{I}$ \\
\hline & 133 & $\mathrm{C}_{14} \mathrm{H}_{10}$ & phenanthrene & $49.0 \pm 3.1$ & $55.8 \pm 3.1$ & $2840.9 \pm 3.1$ & \\
\hline & 134 & $\mathrm{C}_{14} \mathrm{H}_{20}$ & diamantane & $-37.7 \pm 2.1$ & $-20.3 \pm 2.0$ & $3433.4 \pm 2.0$ & I \\
\hline & 135 & $\mathrm{C}_{14} \mathrm{H}_{30}$ & $n$-tetradecane & $-77.9 \pm 4.0$ & $-60.0 \pm 3.9$ & $3989.4 \pm 3.9$ & \\
\hline & 136 & $\mathrm{C}_{15} \mathrm{H}_{32}$ & $n$-pentadecane & $-82.8 \pm 4.2$ & $-63.6 \pm 4.2$ & $4266.3 \pm 4.2$ & \\
\hline & 137 & $\mathrm{C}_{16} \mathrm{H}_{8}$ & [4]circulene & $195.4 \pm 2.6$ & $200.9 \pm 2.6$ & $2932.7 \pm 2.6$ & I \\
\hline & 138 & $\mathrm{C}_{16} \mathrm{H}_{10}$ & fluoranthene & $68.2 \pm 3.4$ & $74.9 \pm 3.4$ & $3161.9 \pm 3.4$ & \\
\hline & 139 & $\mathrm{C}_{16} \mathrm{H}_{10}$ & pyrene & $53.8 \pm 2.9$ & $60.7 \pm 2.9$ & $3176.1 \pm 2.8$ & I \\
\hline & 140 & $\mathrm{C}_{16} \mathrm{H}_{12}$ & [2.2]paracyclophanediene & $123.8 \pm 3.4$ & $131.9 \pm 3.4$ & $3208.2 \pm 3.3$ & \\
\hline & 141 & $\mathrm{C}_{16} \mathrm{H}_{16}$ & {$[2.2]$ metacyclophane } & $41.1 \pm 3.4$ & $53.0 \pm 3.3$ & $3493.6 \pm 3.3$ & \\
\hline & 142 & $\mathrm{C}_{16} \mathrm{H}_{16}$ & [2.2]metaparacyclophane & $48.8 \pm 3.3$ & $60.8 \pm 3.3$ & $3485.8 \pm 3.3$ & \\
\hline & 143 & $\mathrm{C}_{16} \mathrm{H}_{16}$ & [2.2]paracyclophane & $59.7 \pm 3.4$ & $71.4 \pm 3.4$ & $3475.3 \pm 3.4$ & \\
\hline & 144 & $\mathrm{C}_{16} \mathrm{H}_{34}$ & $n$-hexadecane & $-87.6 \pm 4.5$ & $-67.3 \pm 4.5$ & $4543.3 \pm 4.5$ & \\
\hline & 145 & $\mathrm{C}_{18} \mathrm{H}_{12}$ & benzo[c]phenanthrene & $69.8 \pm 3.8$ & $78.0 \pm 3.8$ & $3602.1 \pm 3.8$ & \\
\hline & 146 & $\mathrm{C}_{18} \mathrm{H}_{12}$ & chrysene & $64.0 \pm 4.1$ & $71.9 \pm 4.0$ & $3608.2 \pm 4.0$ & \\
\hline & 147 & $\mathrm{C}_{18} \mathrm{H}_{12}$ & naphthacene & $75.7 \pm 4.0$ & $83.7 \pm 4.0$ & $3596.4 \pm 4.0$ & \\
\hline & 148 & $\mathrm{C}_{18} \mathrm{H}_{12}$ & triphenylene & $63.6 \pm 4.2$ & $71.3 \pm 4.2$ & $3608.9 \pm 4.2$ & \\
\hline & 149 & $\mathrm{C}_{18} \mathrm{H}_{18}$ & 2,4,5,7-tetramethylphenanthrene & $31.5 \pm 3.6$ & $43.2 \pm 3.6$ & $3946.7 \pm 3.6$ & \\
\hline
\end{tabular}


Table S10, contd.

\begin{tabular}{|c|c|c|c|c|c|c|}
\hline MID & & Molecule & $\Delta H_{f}^{0}[M, 298.15 K]$ & $\Delta H_{f}^{0}[M, 0 K]$ & $E_{A, 0}[M]$ & Note \\
\hline 150 & $\mathrm{C}_{18} \mathrm{H}_{18}$ & 2,5-diphenyl-1,5-hexadiene & $65.6 \pm 3.9$ & $77.5 \pm 3.8$ & $3912.4 \pm 3.8$ & \\
\hline 151 & $\mathrm{C}_{18} \mathrm{H}_{18}$ & 3,4,5,6-tetramethylphenanthrene & $32.3 \pm 3.7$ & $44.4 \pm 3.7$ & $3945.5 \pm 3.7$ & \\
\hline 152 & $\mathrm{C}_{20}$ & $\mathrm{C}_{20}$ fullerene & $564.0 \pm 3.8$ & $563.7 \pm 3.8$ & $2836.9 \pm 3.8$ & I \\
\hline 153 & $\mathrm{C}_{20} \mathrm{H}_{10}$ & corannulene & $115.4 \pm 4.1$ & $122.4 \pm 4.1$ & $3794.5 \pm 4.1$ & \\
\hline 154 & $\mathrm{C}_{20} \mathrm{H}_{12}$ & perylene & $75.2 \pm 4.5$ & $83.0 \pm 4.5$ & $3937.1 \pm 4.5$ & \\
\hline 155 & $\mathrm{C}_{20} \mathrm{H}_{14}$ & triptycene & $78.1 \pm 4.1$ & $88.0 \pm 4.1$ & $4035.4 \pm 4.1$ & \\
\hline 156 & $\mathrm{C}_{20} \mathrm{H}_{20}$ & dodecahedrane & $24.0 \pm 4.1$ & $43.1 \pm 4.1$ & $4390.2 \pm 4.1$ & \\
\hline 157 & $\mathrm{C}_{20} \mathrm{H}_{20}$ & pagodane & $52.3 \pm 4.0$ & $70.4 \pm 4.0$ & $4362.8 \pm 4.0$ & \\
\hline 158 & $\mathrm{C}_{20} \mathrm{H}_{36}$ & tetra-tert-butyltetrahedrane & $12.5 \pm 4.9$ & $36.6 \pm 4.8$ & $5222.8 \pm 4.8$ & $\mathrm{~B}_{3}$ \\
\hline 159 & $\mathrm{C}_{24} \mathrm{H}_{12}$ & coronene & $69.7 \pm 5.1$ & $77.9 \pm 5.1$ & $4622.4 \pm 5.1$ & \\
\hline 160 & $\mathrm{C}_{28} \mathrm{H}_{14}$ & [7]circulene & $134.5 \pm 6.0$ & $143.8 \pm 6.0$ & $5339.9 \pm 6.0$ & $\mathrm{~B}_{3}$ \\
\hline 161 & $\mathrm{C}_{32} \mathrm{H}_{16}$ & [8]circulene & $192.4 \pm 6.7$ & $203.0 \pm 6.7$ & $6064.1 \pm 6.7$ & $\mathrm{~B}_{3}$ \\
\hline
\end{tabular}

${ }^{a}$ Molecules are identified by their molecule index MID as specified in Table S1. BMBOD = 3,4-bis(methylene)bicyclo[4.2.0]octa-1,5diene. ${ }^{b}$ Italic fonts indicate theoretical data questioned based on large excess $T_{1}$ diagnostics (see text). ${ }^{c}$ Enthalpies of formation $\Delta H_{f}^{0}[M, 298.15 K]$ are those reported as best estimates in Table 3 if available; otherwise they refer to values reported as ATOMIC(hc) in that same Table. The employed level of theory is completely specified by the label in column "Note" (see Table 3 for details; no label means ATOMIC(hc)/A). Columns $\Delta H_{f}^{0}[M, 0 K]$ and $E_{A, 0}[M]$ report the enthalpy of formation for $T=0 \mathrm{~K}$ and the ZPE-inclusive atomization energy using the same level of theory. A precise definition for the latter quantity and results for all standard models $\left(A, B_{n}\right.$, $\mathrm{C}, \mathrm{E}_{\mathrm{CCSD}}$ ) are listed in Table S9. 


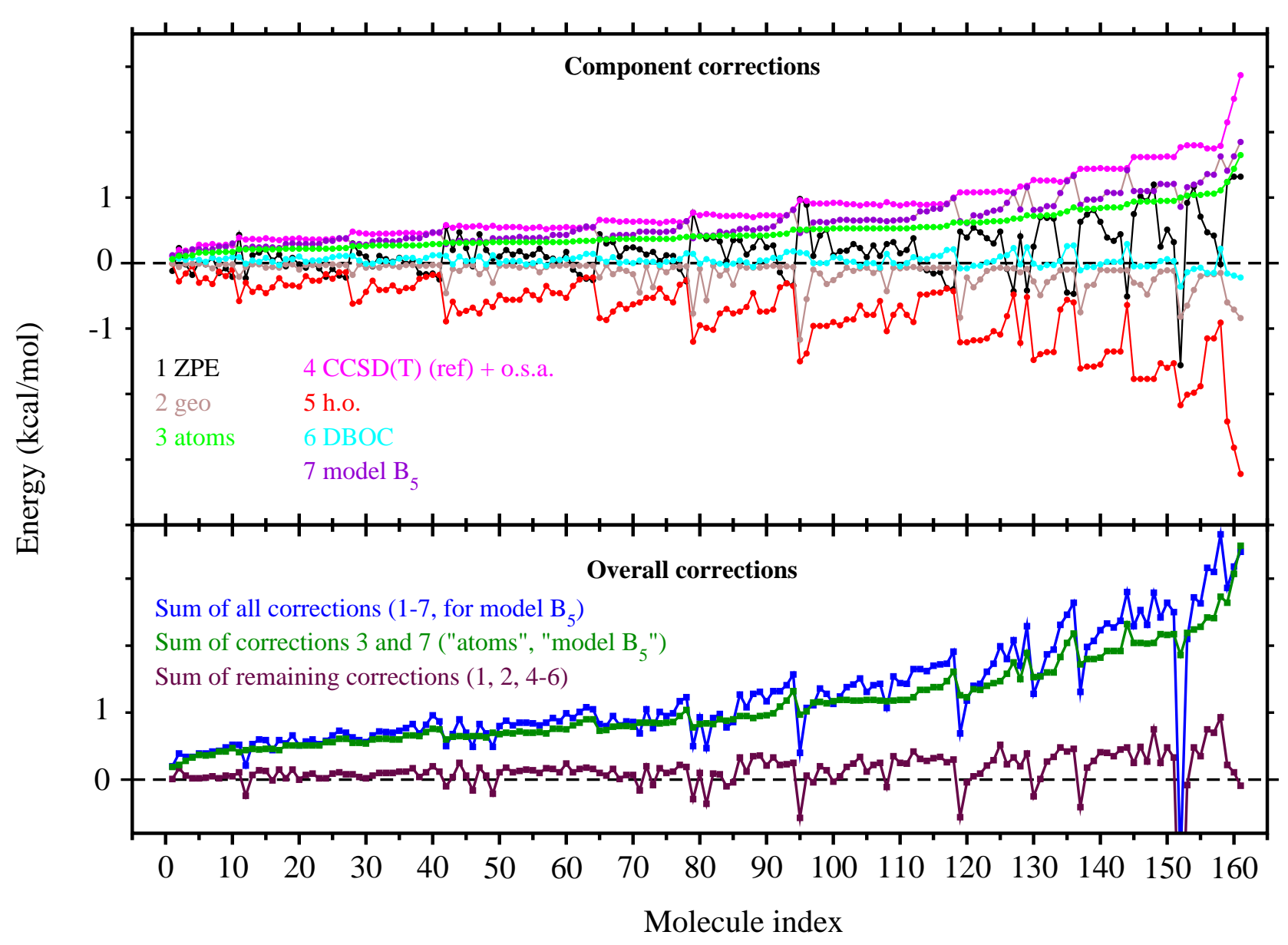

Figure S5: Component corrections for the complete set of 161 hydrocarbons.

The top graph plots ATOMIC(hc) corrections for each of the contributing components and uses the same color coding as the top graph of Fig. 5 uses for component uncertainties. Thermal enthalpy contributions are omitted (no corrections considered, Ref. 138), as are scalar relativistic contributions (no corrections defined), and low-level CCSD(T) corrections for model $\mathrm{B}_{5}$ are added (in violet). The bottom graph sums over all corrections defined for ATOMIC(hc) (blue, same as lower curve in bottom graph of Fig. 5) or two mutually exclusive subsets (green, maroon). 